\title{
SURFACE PLASMON RESONANCE SENSOR BASED ON THE TILTED FIBER BRAGG GRATING
}

\author{
By \\ Yanina Shevchenko \\ B Eng., Saint-Petersburg, Russia, 2004
}

A Thesis Submitted to the Faculty of Graduate Studies and Research in Partial Fulfillment of the Requirements for the Degree Master of Applied Science

Ottawa-Carleton Institute for Electrical and Computer Engineering Department of Electronics

Carleton University

Ottawa, Ontario, Canada

September 2007

CYanina Shevchenko 


$\begin{array}{ll}\begin{array}{l}\text { Library and } \\ \text { Archives Canada }\end{array} & \begin{array}{l}\text { Bibliothèque et } \\ \text { Archives Canada }\end{array} \\ \begin{array}{l}\text { Published Heritage } \\ \text { Branch }\end{array} & \begin{array}{l}\text { Direction du } \\ \text { Patrimoine de l'édition }\end{array} \\ \begin{array}{l}\text { 395 Wellington Street } \\ \text { Ottawa ON K1A ON4 }\end{array} & \begin{array}{l}\text { 395, rue Wellington } \\ \text { Ottawa ON K1A ON4 } \\ \text { Canada }\end{array}\end{array}$

Your file Votre référence ISBN: 978-0-494-33667-0 Our file Notre référence ISBN: 978-0-494-33667-0

NOTICE:

The author has granted a nonexclusive license allowing Library and Archives Canada to reproduce, publish, archive, preserve, conserve, communicate to the public by telecommunication or on the Internet, loan, distribute and sell theses worldwide, for commercial or noncommercial purposes, in microform, paper, electronic and/or any other formats.

The author retains copyright ownership and moral rights in this thesis. Neither the thesis nor substantial extracts from it may be printed or otherwise reproduced without the author's permission.
AVIS:

L'auteur a accordé une licence non exclusive permettant à la Bibliothèque et Archives Canada de reproduire, publier, archiver, sauvegarder, conserver, transmettre au public par télécommunication ou par l'Internet, prêter, distribuer et vendre des thèses partout dans le monde, à des fins commerciales ou autres, sur support microforme, papier, électronique et/ou autres formats.

L'auteur conserve la propriété du droit d'auteur et des droits moraux qui protège cette thèse. $\mathrm{Ni}$ la thèse ni des extraits substantiels de celle-ci ne doivent être imprimés ou autrement reproduits sans son autorisation.
In compliance with the Canadian

Privacy Act some supporting forms may have been removed from this thesis.

While these forms may be included in the document page count, their removal does not represent any loss of content from the thesis.
Conformément à la loi canadienne sur la protection de la vie privée, quelques formulaires secondaires ont été enlevés de cette thèse.

Bien que ces formulaires aient inclus dans la pagination, il n'y aura aucun contenu manquant.

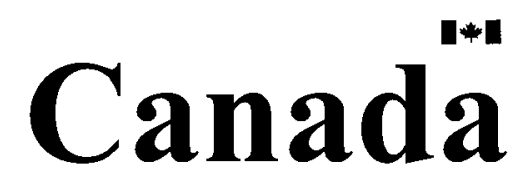




\begin{abstract}
This thesis proposed a new Surface Plasmon Resonance (SPR) fiber-based sensor. The sensor was realized in an un-modified standard single mode optical fiber with a Tilted Fiber Bragg Grating (TFBG) written in its core and a gold coating deposited on the surface of the fiber. TFBG is a photo-induced variation of the refractive index of the fiber's core, which enables coupling of the forward propagating core mode to the backpropagating core mode and a set of back-propagating cladding modes. SPR was excited on the surface of the gold-coated fiber through an interaction of evanescent fields of these cladding modes with the metal coating. If the wave vector of one of the cladding modes matches the wave vector of the surface plasmon wave, which can exist on the metal's surface, the plasmon wave is excited on the outer metal's surface.

The focus of this research was to determine if the gold-coated TFBG could be used to excite SPR. Simple calculations employing theory developed for TFBG and prism-based SPR configuration showed that SPR could be excited in the single mode fiber with TFBG without a need to reduce the cladding diameter. These calculations were used to predict an operating range for the proposed sensor and its sensitivity. Experiments proved that it was possible to excite SPR using the proposed sensor configuration. The sensitivity obtained for the sensor varied from $420.5 \mathrm{~nm} /$ refractive index unit (r.i.u.) to $539 \mathrm{~nm} / \mathrm{r}$.i.u. depending on the metal coating thickness, which is very close to the predicted sensitivity that is considered to be high among other SPR fiber-based sensors.
\end{abstract}




\section{Acknowledgements}

I would first like to thank Professor Michel Nakhla and Professor Jacques Albert for providing me with funding to pursue a Masters degree at Carleton University. I would also like to thank the Government of Ontario for providing additional support through the Ontario Graduate Scholarship (OGS) Program.

I am very grateful to my supervisor Jacques Albert for giving me an exciting project and his guidance throughout it and particularly during the process of writing this thesis. He was patient with questions and always available to answer them in a highly professional manner. His help and guidance are greatly appreciated.

I would like to thank Albane Laronche for helping me in the lab with equipment preparations and troubleshooting any unforeseen problems that occurred during the experiments. I am grateful to Rob Vandusen for spending a lot of time in the Fabrication Laboratory helping me with the fabrication of the sensor and teaching me how to use the different equipment. The experimental process would have taken a lot longer without their help. I am thankful to Professor Anatolii Ianoul for helping us acquire AFM images.

I am very grateful to Rodica Rogojan for her discussions and innovative thinking that helped solve some problems throughout the various stages of this research. I would also like to thank the other members of the Laboratory for Laser Induced Photonic Structures: Alexander Andreyuk, Lingyun Xiong, Alexei Ivanov, Ksenia Yadav and Chengkun Chen. All these people have created a warm environment in lab to help aid in constructive research through open discussions.

I offer my gratitude to Lidija Filipovic for spending night hours in the CAD Lab debugging my code and for offering all other help during my first days at Carleton University. I am very thankful to Ksenia Yadav for her friendship, great support and enthusiasm on many occasions. I am also thankful to Natalia Yastrebova for her friendship over the years of my study and always making things less stressful.

In the end I would like to say thank you to my family for love and support during my endless studies and express my deep gratitude to my father for inspiring my interest in the natural sciences. Last and foremost, I want to thank my husband and best friend, Robert for his love, patience and substantial help during all this time. 


\section{Table of Contents}

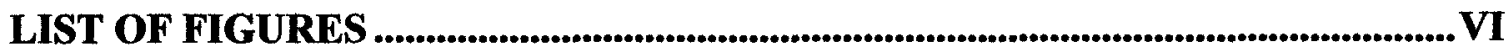

LIST OF ACRONYMS ................................................................................................ VIII

CHAPTER 1. INTRODUCTION ........................................................................... 1

CHAPTER 2. FIBER GRATING ................................................................................... 4

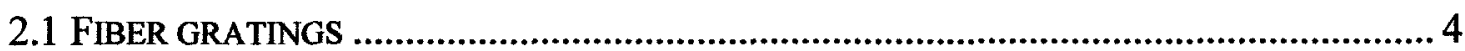

2.2 THE FUNDAMENTAL MECHANISMS BEHIND PHOTOSENSITIVITY ............................... 5

2.3 DIVERSITY OF THE FIBER GRATINGS AND THEIR APPLICATIONS ............................... 7

2.4 THE MECHANISM OF LIGHT DIFFRACTION IN FIBER GRATING ................................. 8

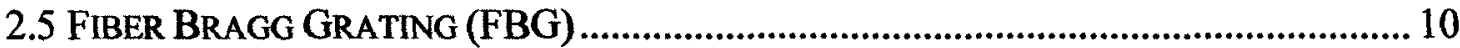

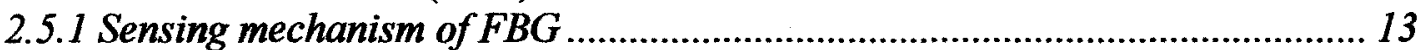

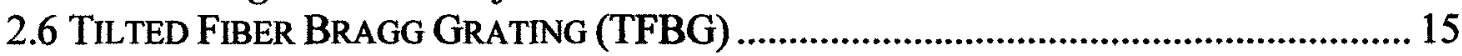

2.6.1 Sensing mechanism of TFBG ............................................................ 18

CHAPTER 3. SURFACE PLASMON RESONANCE ......................................... 21

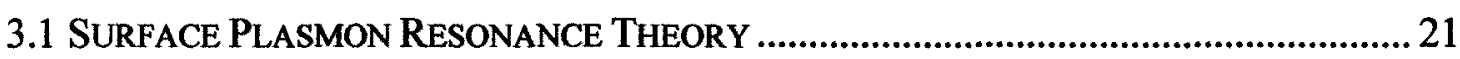

3.1.1 Electromagnetic field propagation in the slab waveguide........................... 22

3.1.2 Field distribution in the slab waveguide ....................................................... 24

3.1.3 SPW at the interface between semi-infinite mediums ................................. 24

3.1.4 Characteristics of SPW ................................................................................ 26

3.1.5 Propagation of SPW at the dielectric-metal-dielectric interface.................... 29

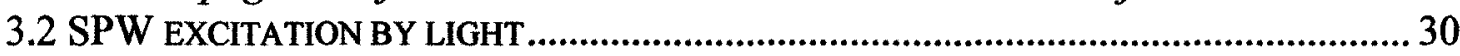

3.2.1 Prism configuration ............................................................................... 31

3.2.1.1. Absorption-based SPR sensing theory .................................................... 34

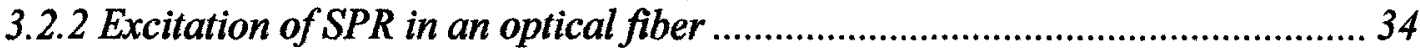

CHAPTER 4. PROPOSED SENSOR DESIGN......................................................... 42

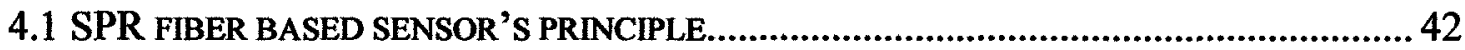

4.2 CALCULATION OF THE SPR PARAMETERS AND EXCITATION CONDITIONS ................. 44

4.2.1 Refractive index of sensed medium $n_{d}$ and operating range $\Delta n_{d} \ldots \ldots \ldots \ldots \ldots . . . . . . .44$

4.2.2. Proposed sensitivity ................................................................................. 46

4.2.3 SPW penetration depth into sensed medium $L_{p d}$ metal $L_{p m}$ and length of

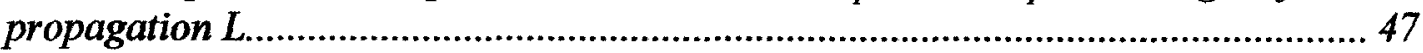

4.2.4 Optimal metal thickness calculations ........................................................ 47

CHAPTER 5. MANUFACTURING OF THE SPR SENSOR..............................50

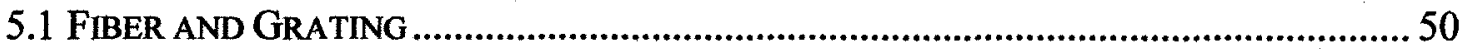

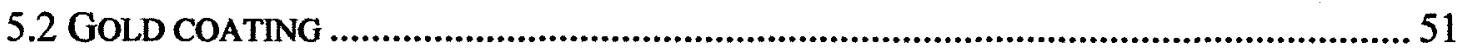

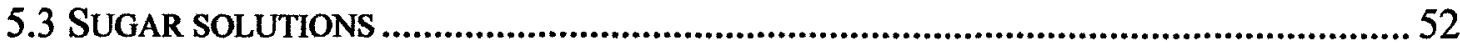

CHAPTER 6. EXPERIMENTAL RESULTS ............................................................5 54 


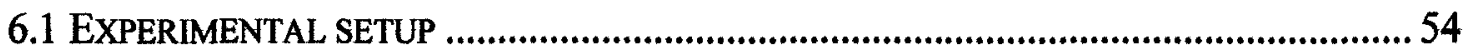

6.2 EXPERIMENTAL RESULTS FOR GRATING WITH A 20 NM THICK GOLD COATING ......... 55

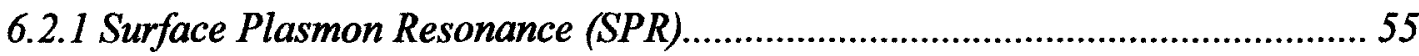

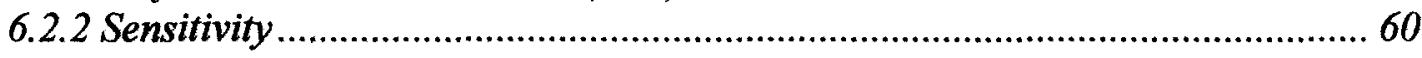

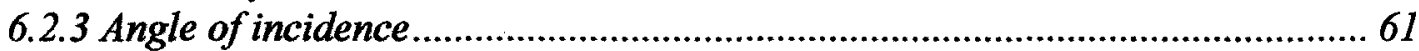

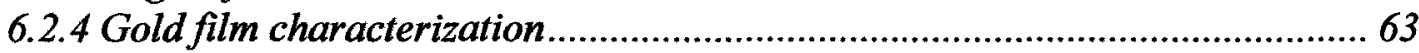

6.3 EXPERIMENTAL RESULTS FOR SENSOR WITH 30 AND 50 NM OF THE GOLD LAYER

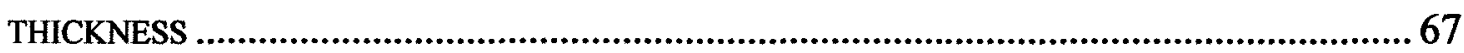

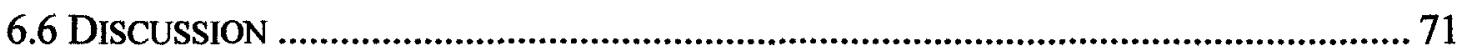

CHAPTER 7. CONCLUSION AND FUTURE WORK RECOMMENDATIONS.. 76

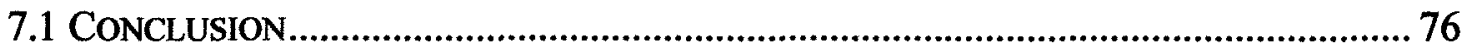

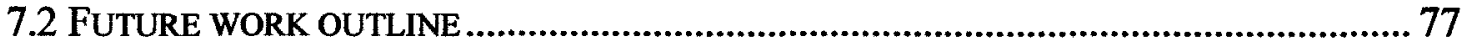

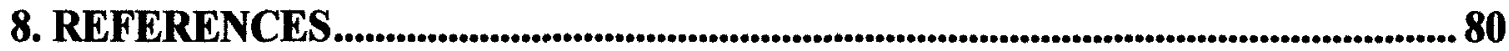




\section{List of Figures}

Figure 2.1. Different types of fiber gratings including (a) uniform with positive-only index change, (b) Gaussian-apodized, (c) raised-cosine-apodized with zero-dc index change, (d) chirped, (e) discrete phase shift (of $\pi$ ), and (f) superstructure. The effective refractive index change is proportional to the core refractive index change $\Delta n_{e f f} \cong \Gamma \Delta n_{c o}$ (in the case when only the core is photosensitive) where $\Gamma$ is the core power confinement factor for the mode of interest [Erdogan, 1997]........................ 7

Figure 2.3. Vector diagram of light scattering in the Bragg grating.............................. 10 Figure 2.4. Reflection spectra versus normalized wavelength for Bragg reflection in FBG with $k L=2$ and $k L=8$ ( $k$ is the wave number) [Erdogan, 1997] ........................... 12

Figure 2.5. Vector diagram of light diffraction in the Tilted Fiber Bragg Grating........... 15

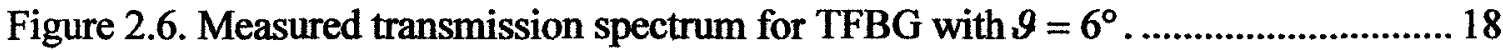

Figure 3.1. Planar waveguide with step refractive index profile. ................................. 22

Figure 3.2. Interface between semi-infinite mediums with permittivity $\varepsilon_{1}$ and $\varepsilon_{2} \ldots \ldots \ldots . .25$

Figure 3.3. Distribution of the magnetic field intensity for an SPW at the interface between gold and dielectric (refractive index of the dielectric 1.32) in the direction perpendicular to the interface calculated for two different wavelengths [Homola, 2003] 28

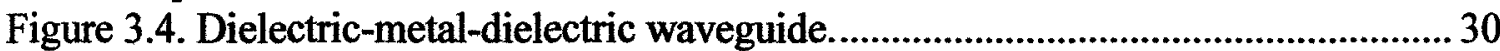

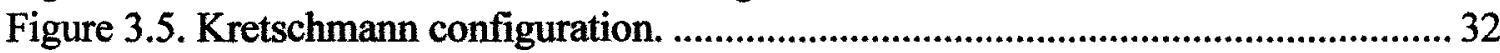

Figure 3.6. Reflectivity for light wave exciting an SPW in the geometry versus

wavelength for two different refractive indices of the dielectric (angle of incidence is

$54^{\circ}$ ) [Homola, 2003].

Figure 3.7. Diagram showing fiber SPR sensors working in the transmission regime (a) and reflection regime $(b)$. 36

Figure 3.8 SPR sensor based on the fiber with deep cut in the cladding [Jorgenson and

Yee, 1993] 37

Figure 3.9. SPR sensor based on the bent and side-polished fiber [Homola, 1995]........ 38

Figure 3.10. SPR sensor based on the tapered fiber [Tubb et al., 1997].......................... 38

Figure 3.11 SPR sensor based on the fiber with LPG [He et al., 2006]............................ 39

Figure 3.12. SPR sensor based on the hollow core fiber with FBG [Nemova and Kashyap, $2006]$.

Figure 4.1. Schematic diagram of the proposed SPR sensor to show the principle of light

coupling through TFBG into cladding modes and SPWs, consecutively. 43

Figure 4.2. Transmission spectrum of the grating that was used in experiments (tilt angle

is $4^{\circ}$ )

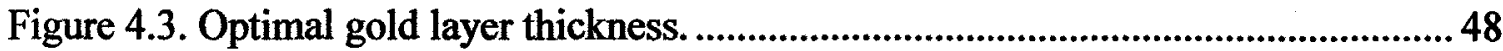

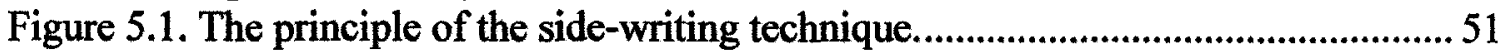

Figure 5.2. Principle of gold deposition in the chamber .............................................. 52

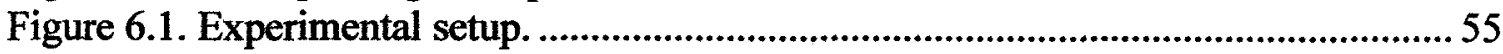

Figure 6.2. Transmission spectrum of the gold coated grating with a $4^{\circ}$ tilt measured in

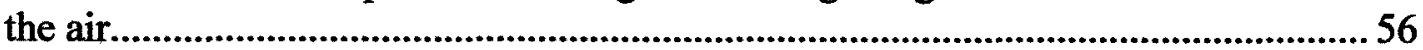

Figure 6.3. Transmission spectrum for the gold coated fiber immersed in the sugar solution with a refractive index of $n_{d}=1.4378$.

Figure 6.4. Transmission spectrum for an uncoated fiber immersed in the sugar solution with a refractive index of $n_{d}=1.4378$. 
Figure 6.5. Transmission spectrum of the same grating as in Figure 6.3 immersed in sugar

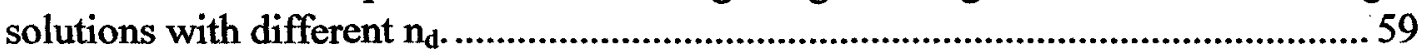

Figure 6.6. Transmission spectrum for grating immersed in the sugar solution with

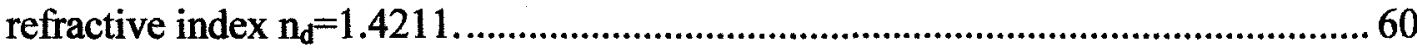

Figure 6.7. Dependence of the plasmon resonance peak wavelength $(\lambda p)$ on the refractive

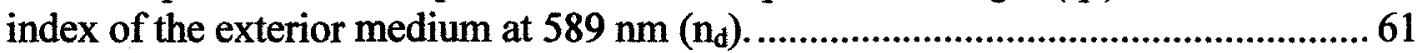

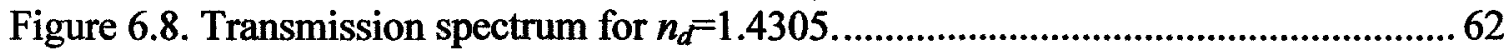

Figure 6.9. Transmission loss versus angle of incidence $\theta$ for $\mathrm{n}_{\mathrm{d}}=1.4378$......................6. 63

Figure 6.10. SEM images of TFBG fiber with gold coating (nominal thickness $20 \mathrm{~nm}$ ) before experiments

Figure 6.11. SEM images of TFBG fiber with gold coating $(20 \mathrm{~nm})$ after 10 experiments in sugar solutions [Shevchenko et al., 2007]. ....................................................6 65

Figure 6.12. SEM images of TFBG fiber with gold coating $(20 \mathrm{~nm})$ after intense testing in sugar solutions (approximately 30 experiments). 65

Figure 6.13. AFM Section Analysis of the gold coating near an edge where mechanical abrasion was used to partially remove the coating. The vertical distance between red cursors is $26.783 \mathrm{~nm}$; horizontal distance between red cursors is $218.75 \mathrm{~nm}$ [Shevchenko et al., 2007] 67

Figure 6.14. Transmission spectra for grating with gold thickness of $30 \mathrm{~nm}$ immersed in different sugar solutions (tilt angle is $6^{\circ}$ ).

Figure 6.15. Transmission spectra for grating with gold thickness of $50 \mathrm{~nm}$ immersed in different sugar solutions (tilt angle is $6^{\circ}$ ) 


\section{List of Acronyms}

$\begin{array}{ll}\text { AFM } & \text { Atomic Force Microscope } \\ \text { FBG } & \text { Fiber Bragg Grating } \\ \text { LPG } & \text { Long-Period Grating } \\ \text { r.i.u. } & \text { refractive index unit } \\ \text { SEM } & \text { Scanning Electron Microscope } \\ \text { SPP } & \text { Surface Plasmon-Polariton } \\ \text { SPR } & \text { Surface Plasmon Resonance } \\ \text { SPW } & \text { Surface Plasmon Wave } \\ \text { TE } & \text { Transverse Electric } \\ \text { TFBG } & \text { Tilted Fiber Bragg Grating } \\ \text { TIR } & \text { Total Internal Reflection } \\ \text { TM } & \text { Transverse Magnetic }\end{array}$

viii 


\section{Chapter 1. Introduction}

A Surface Plasmon Resonance (SPR) is an electromagnetic phenomenon occurring as a resonant energy transfer of light into a bound surface mode propagating at the interface between a metal and a dielectric. The unique physical properties of SPR have attracted a lot of attention in recent years in optical research communities. Originally used for the thin film characterization [Pockrand et al., 1978], it has been proposed for use in such areas as solar cells [Schewe and Stein, 2007], medical therapy [O'Neal et al., 2004] and medical imaging [Loo et al., 2005], design of miniaturized photonic circuits [Barnes et al., 2003] and also in a variety of sensing applications in medicine, biochemistry and environmental disciplines.

Most SPR sensing applications occur because a Surface Plasmon Wave (SPW) is confined in a very small volume at the interface between materials. The depth of SPW penetration in mediums varies within several hundreds of $\mathrm{nm}$, and SPR is very sensitive to any change occurring within the distance of penetration. Small variations in the properties of the material result in a change of the propagation constant of SPW. As a result SPR can be used to measure such parameters as complex refractive index, thickness of the materials and their dynamics.

In comparison to other optical sensing technologies, SPR offers a surfacesensitive response and the possibility to make real-time kinetic measurement. One of the main advantages of SPR is that there is no need for labeling. Labeling is a technique that employs a fluorescent, luminescent, radiometric or colorimetric factor on the surface of the biosensing element in order to generate or significantly increase sensor signal [Wang et al, 1997]. This technology seems to be one of the main procedural problems in the biosensing field and pushes the need for label-free technology [Endo et al., 2005]. However the sensitivity of the SPR technique is less than the sensitivity obtainable with labeled sensors [Ince and Narayanaswamy, 2006]. This fact would be greatly compensated if SPR technology would provide simpler and more compact sensors. However the previous generation of sensors based on SPR utilized the prism configuration and were complicated (required moving parts), bulky and costly. Recent 
developments in this area led to creating more compact sensors by replacing the prism by an optical fiber. This initially resulted in a drop of sensitivity [Jorgenson and Yee, 1993; Homola and Slavik, 1996; Tubb et al., 1997], and still the sensitivity offered by the prism configuration is not achievable for many fiber SPR sensors.

To achieve the SPR effect, the first fiber sensors were based on non-standard and modified fibers. Fiber that is deformed is able to redirect the optical field of the cladding and core modes to the metal coating on the fiber's surface where the SPR could be excited. High compactness was achieved by sacrificing the sensitivity and robustness. Further developments in this field involved reducing fragility while increasing control of the SPR effect, which resulted in an increase of the sensors precision. The most recent generation of SPR fiber-based sensors utilize fiber gratings. Fiber gratings provide a great opportunity to achieve the same effect as deformation of the fiber by delivering optical fields to the metal layer deposited on the fiber's surface, but without damaging the fiber's integrity.

In this thesis we investigate the possibility to integrate SPR and fiber grating technology (Tilted Fiber Bragg Gratings (TFBG) to be specific) to achieve an extremely simple and efficient sensor embedded in the optical fiber. This work presents a new SPR fiber-based sensor where the SPR is being excited on the gold-coated fiber's surface via coupling of the core mode to cladding modes and their further coupling to the SPW. The sensor offers high sensitivity, relatively high operating range and the possibility to tune it over wide range of refractive indices of the sensed medium. The sensor itself is very compact, its fabrication is easy and implementation is simple. The design does not require any deformations as presented in many proposed SPR fiber-based sensors.

The research presented here includes calculations to determine such sensor's characteristics as sensitivity, optimal gold layer thickness and operating range. The sensor was fabricated and tested in various solutions in order to characterize SPR behavior over the operating range. Validity of the gold thickness calculations was verified during the testing of the several sensors with different thickness of the gold layer. Gold surface quality and uniformity were investigated with the aid of an Atomic Force Microscope (AFM) and a Scanning Electron Microscope (SEM). 
This thesis is organized as follows. Chapter 2 presents background material relating to the fiber grating technology and literature review of fiber Bragg-grating based sensors. SPR theme is covered in Chapter 3 where theoretical information and a literature review of SPR fiber sensors are provided. Chapter 4 introduces the proposed SPR sensor and shows simple calculations to determine the sensor's main parameters. Manufacturing process of the proposed sensor is discussed in Chapter 5. Experimental results along with gold film characterization are presented in Chapter 6. This chapter is concluded with a detailed discussion of the results along with the sensor's advantages and limitations. Chapter 7 summarizes the work conducted in this thesis and presents a future work outline. 


\section{Chapter 2. Fiber grating}

\section{Objectives:}

The fiber grating is considered to be the key element in the proposed sensor design since it enables excitation of SPR on the fiber's surface. The main objective of Chapter 2 is to introduce the fundamentals of fiber grating technology. Section 2.1 begins with a definition of fiber grating and provides a short history and background of this field. Section 2.2 provides an introduction regarding the fundamental processes behind the photosensitivity mechanism. Sections 2.3 covers a variety of modern gratings and their applications. The principle of light diffraction in the fiber grating is presented in Section 2.4. Section 2.5 discusses Fiber Bragg Grating (FBG) and their sensing applications. Section 2.6 introduces TFBG with a description of its applications with emphasis on the sensing.

\subsection{Fiber gratings}

Typically, a fiber grating consists of a periodic perturbation of the refractive index of fiber core $n_{\text {core }}$ [James and Tatam, 2003] (sometimes the perturbation can also be aperiodic) and can be presented as:

$$
n_{\text {core }}(z)=n_{0}+\Delta n \cos \left(\frac{2 \pi z}{\Lambda}\right)
$$

where $\Lambda$ is the spatial period of perturbations, $\Delta n$ is the amplitude of induced refractive index perturbations and $\mathrm{z}$ is the distance along the fiber longitudinal axis.

Hill and coworkers in 1978 'accidentally' discovered the effects of photosensitivity in optical fibers, as well as, the first gratings in the optical fiber [Hill et al., 1978]. This occurred when argon laser radiation at $488 \mathrm{~nm}$ and $514.5 \mathrm{~nm}$ was launched into a germanium-doped silica fiber. An interferometric pattern formed by contra-directional propagating waves formed a standing wave pattern in the fiber core, which resulted in permanent periodic perturbations of the fiber core refractive index. Permanent refractive index perturbation became possible because of the photosensitivity effect that is characteristic for silica materials with different dopants. Gratings written with the help of fiber laser radiation launched into the fiber form a standing wave pattern 
known as a self-organizing grating. The application of such gratings was limited because the range of reflected back wavelengths is limited by the wavelength of radiation used to form the interoferomic pattern within the fiber core.

The next step in the development of the fiber grating was the side-writing or transverse holographic technique that allowed the creation of a grating with various periods. Meltz et al, (1989) wrote a grating in the fiber core by illuminating it with an interferometric pattern formed outside the fiber produced by the intersection of two coherent beams at $240-250 \mathrm{~nm}$. The period of the formed grating can be easily changed by varying the angle between the beams which form the pattern, hence the wavelength of the reflected beam can be easily changed and achieve a long wavelength range (1200 $\mathrm{nm}$ $-1500 \mathrm{~nm}$ ). This last condition made this type of grating inscribed in the fiber core very applicable and facilitated their popularization [Othonos and Kalli, 2000].

\subsection{The fundamental mechanisms behind photosensitivity}

The effect of photosensitivity is not completely understood. However, it is well known that photosensitivity of optical fibers would not be possible in silica without different dopants which are ions of such metals as germanium, europium, cerium, erbium and boron [Othonos and Kalli, 1999]. Germanium and germanium-boron are doping elements that enable the fiber material to obtain the highest photosensitivity.

Usually, dopants are used to create a waveguide material with higher refractive index, which can be used as the core material for fibers. The difference created between the refractive indices of the core and cladding - causes light to be guided in the fiber core by means of Total Internal Reflection (TIR) at the core-cladding interface. For example, in standard CORNING SMF 28 telecom fiber, pure silicon dioxide $\mathrm{SiO}_{2}$ is used for cladding, while $\mathrm{SiO}_{2}$ with dopants of $\mathrm{Ge}$ is used for fiber core. Germanium has four valence electrons on the external orbit and can form tetrahedron units with four atoms of oxygen $\left(\mathrm{GeO}_{4}\right)$ in the same way as the silicon atom $\left(\mathrm{SiO}_{4}\right)$ [Battiato, 1998]. The process of manufacturing silica fibers is based on modified chemical vapor deposition method where such gases as oxygen and silica/germanium tetrachloride are getting mixed and dioxides of silicon and germanium are deposited on the inside of a silica support tube [Kashyap, 1999]. Given the nature of the described process, germanium can form not 
only the oxide $\mathrm{GeO}_{4}$, but also any of the suboxides $\mathrm{GeO}_{x}(x=1$ to 4$)$. Suboxides with a number of oxygen atoms less than 4 are considered as a defect and are known as 'color centers'. Defects can be divided into two major groups: in the first case, germanium uses several valence electrons with oxygen atoms and shares the rest of electrons with another atom of germanium or silicon (wrong bonds) [Hosono and Abe, 1992]; in the second case, germanium does not share left electrons and keeps them unbounded. The presence of these defects was identified to cause photosensitivity effect in silica glasses. Although it is not completely explained how the defect influences the process of photosensitivity, it is shown that both types of defects have absorption bands in the UV between $197 \mathrm{~nm}$ and $514.5 \mathrm{~nm}$. Absorption in the UV band relates to single-photon and two-photon absorption processes. Both processes lead to chemical changes in the defects that result in a permanent change of the refractive index of silica glass. While the two-photon absorption process is characteristic for longer wavelengths and helps to inscribe self-induced gratings, single-photon absorption is used at shorter wavelengths and requires a million times less power to produce the same refractive index change as two-photon absorption processes [Grattan et al., 2000].

It was shown that higher concentrations of $\mathrm{Ge}^{4+}$ ions result in a higher photosensitivity of germanosilica glass [Kashyap, 1999]. To achieve an increase in the photosensitivity of standard telecom fibers with concentration of $\mathrm{Ge}^{4+}$ dopants at the level of 3-5\%, several photosensitization methods were developed. Among them such kinds of hydrogenation as hot and cold hydrogenation, high-pressure and low-pressure hydrogenations can be named [Kashyap, 1999].

Hydrogen loading involves the soaking of a fiber in the hydrogen gas prior to the UV exposure [Lemaire et al., 1993]. Illumination of hydrogen loaded fiber by UV leads to dissociation of the $\mathrm{H}_{2}$ molecules and reaction of $\mathrm{H}^{+}$ions with Ge-based defects. Hydrogen loading allows almost every ion of $\mathrm{Ge}+$ to participate in the permanent change of the refractive index, resulting in an increase in the amplitude of induced refractive index change from $3 \times 10^{-5}$ to $10^{-2}$ [Limberger et al., 1993]. 


\subsection{Diversity of the fiber gratings and their applications}

Even though fiber grating development has a history spanning almost 30 years, there is still room to further understand the processes responsible for grating formation. During these 30 years, fiber gratings became an important element in optical technology. Evolution of the fabrication of fiber gratings resulted in a diversity of gratings such as long-period and short-period gratings (Bragg gratings), tilted Bragg gratings, chirped gratings, phase-shifted gratings (Figure 2.1) and a combination of grating designs (superimposed Bragg gratings) [Othonos and Kalli, 2000].

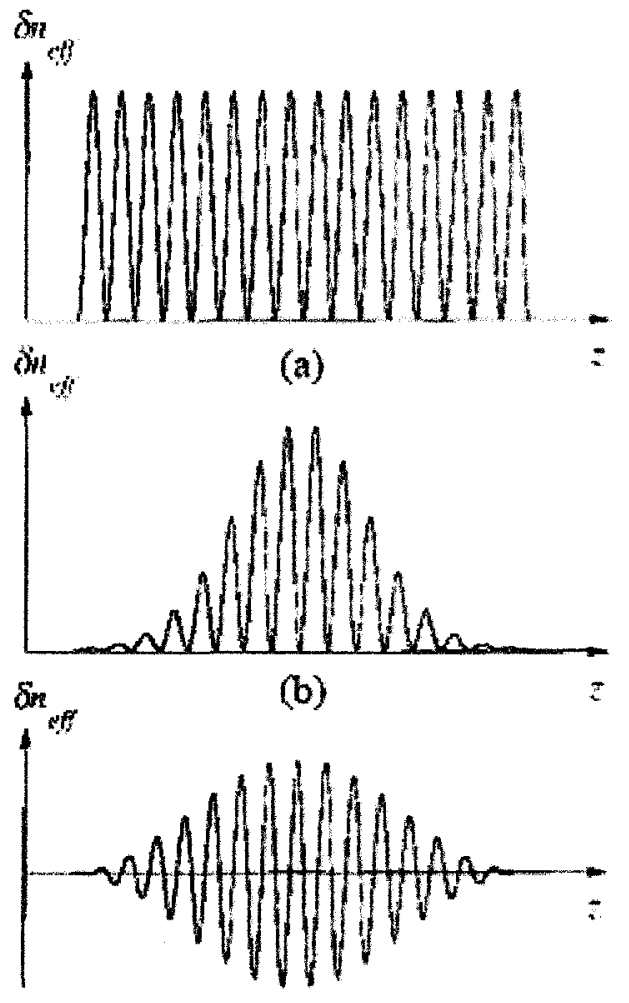

(c)
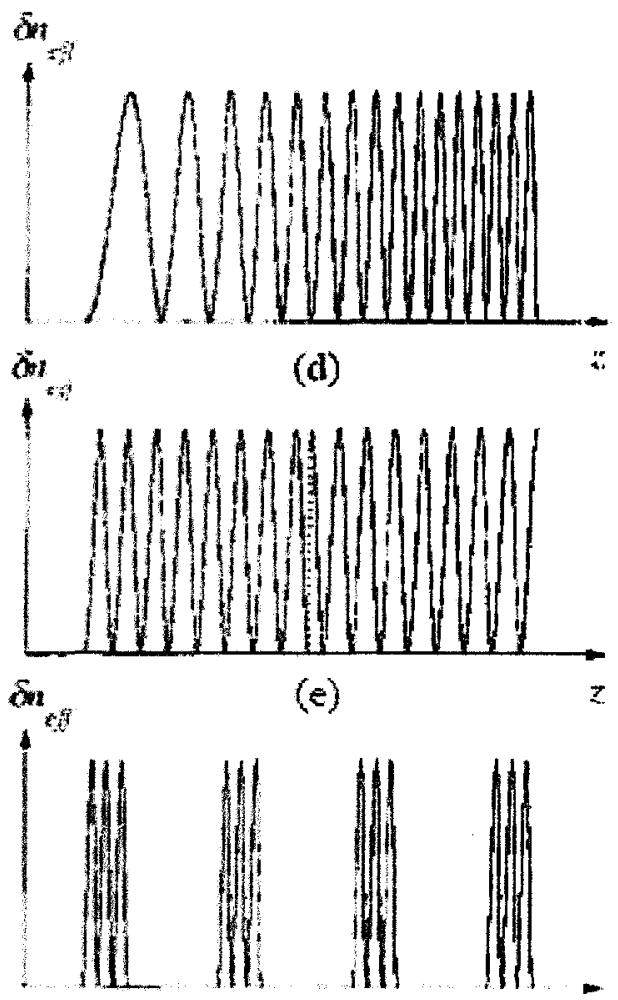

$(0$

Figure 2.1. Different types of fiber gratings including (a) uniform with positive-only index change, (b) Gaussian-apodized, (c) raised-cosine-apodized with zero-dc index change, (d) chirped, (e) discrete phase shift (of $\pi$ ), and (f) superstructure. The effective refractive index change is proportional to the core refractive index change $\Delta n_{e f f} \cong \Gamma \Delta n_{c o}$ (in the case when only the core is photosensitive) where $\Gamma$ is the core power confinement factor for the mode of interest [Erdogan, 1997]. 
Fiber gratings have become a powerful tool in the telecommunication field and result in the appearance and sophistication of already existing devices such as mode couplers [Thiele et al., 2003], dispersion compensators [Loh et al., 1996], narrowband and broadband filters [Psaila and Inglis, 2001] and fiber lasers [Ibsen et al., 2003; Othonos and Kalli, 2000].

Another major application of fiber gratings is in the sensing field. Sensors based on fiber gratings are able to detect change of such parameters as temperature [Zhang and Kahrizi, 2007], bending, microbending, strain [Shu et al., 2002], pressure [Sheng et al., 2004], refractive index [Laffont and Ferdinand, 2001] and have found their niche in such fields as aerospace, biomedicine, marine, civil engineering, nuclear power and oil industries [Fernandez et al., 2006; Othonos and Kalli, 2000].

\subsection{The mechanism of light diffraction in fiber grating}

Scattering of light in the grating imprinted in optical fiber could simply be explained based on the example of light diffraction in the optical diffraction grating. Diffraction of light in the grating can be described simply with the help of a ray-optical analogy of this process. Figure 2.2 shows the scattering of a wave front in a diffraction grating with period $\Lambda$. 


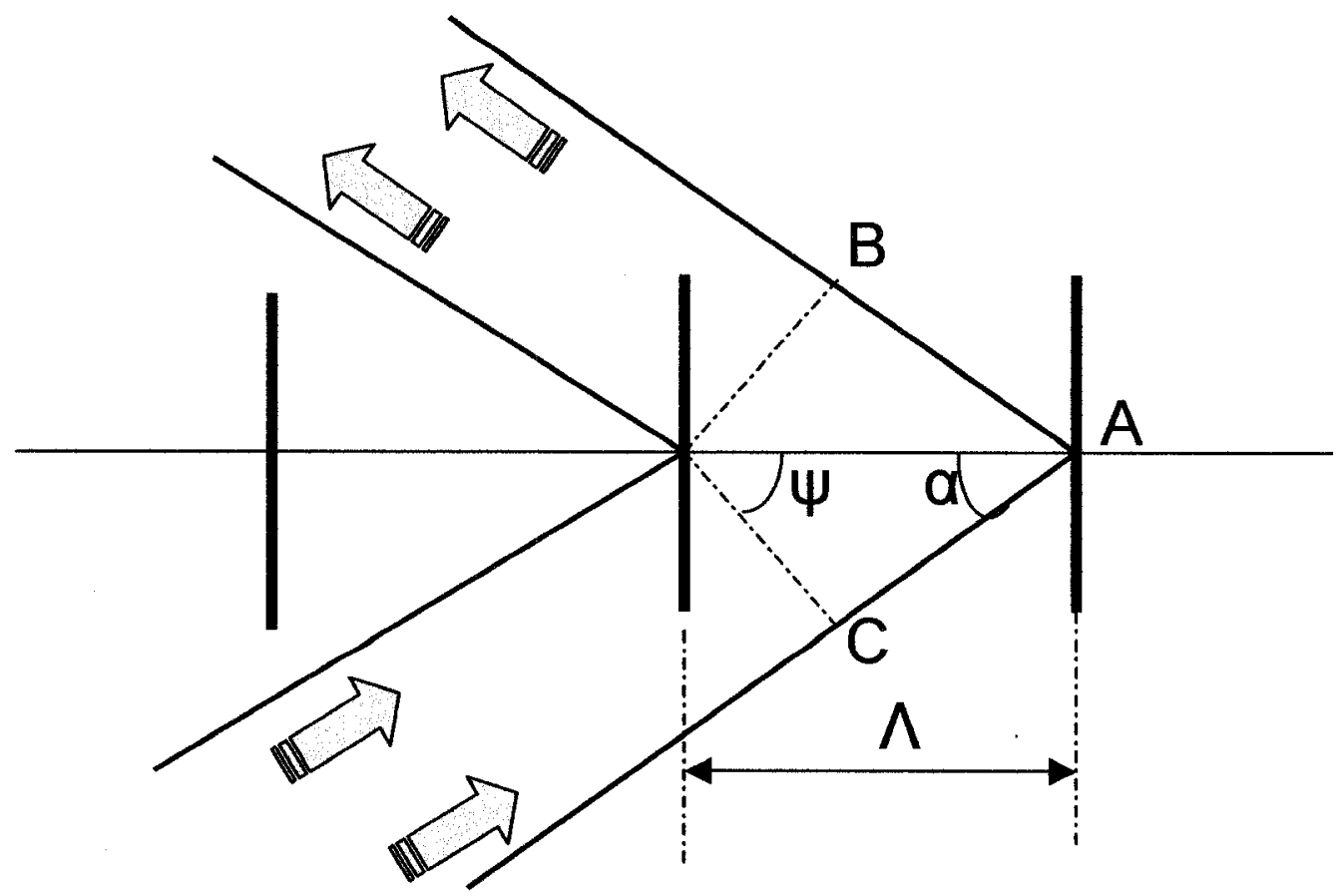

Figure 2.2. Scattering of a wave front in a diffraction grating.

Constructive interference between incoming radiation and scattered radiation can occur only if the pathway of reflected wave $\mathrm{CA}+\mathrm{AB}$ equals to an integral number of wavelengths:

$C A+A B=n \lambda$,

where $n= \pm 1,2 \ldots$ is the diffraction order.

Given that:

$C A=A B=\Lambda \sin (\psi)$,

The condition (2.1) can be rewritten as:

$2 \Lambda \sin (\psi)=n \lambda$,

Based on the assumption that light propagates in the fiber's core with refractive index $n_{\text {core }}$ at an angle $\alpha$ relative to the fiber axis, the effective index of the mode is defined by: $n_{\text {eff }}=n_{\text {core }} \cos (\alpha)$,

then (2.3) is:

$2 n_{e f f}=\frac{n \lambda}{\Lambda}$ 


\subsection{Fiber Bragg Grating (FBG)}

Equation 2.5 is known as the Bragg phase matching condition. If the grating has a period of about one-half of the propagating core mode wavelength, it is considered to be a Bragg grating, also known as short period or reflection grating [Erdogan, 1997]. The phase matching condition also can be derived with the help of coupled wave analysis of light diffraction in the grating (Figure 2.3). Scattering of the incoming light wavevector $K_{i}$ by the grating with wavevector $K_{g}$ obeys momentum conservation which states that the sum of the incoming light wavevector $K_{i}$ and grating wavevector $K_{g}$ should be equal to wavevector of scattered radiation $K_{r}$ [Othonos and Kalli, 2000].

$$
\vec{K}_{i}+\vec{K}_{g}=\vec{K}_{r} \text {, }
$$

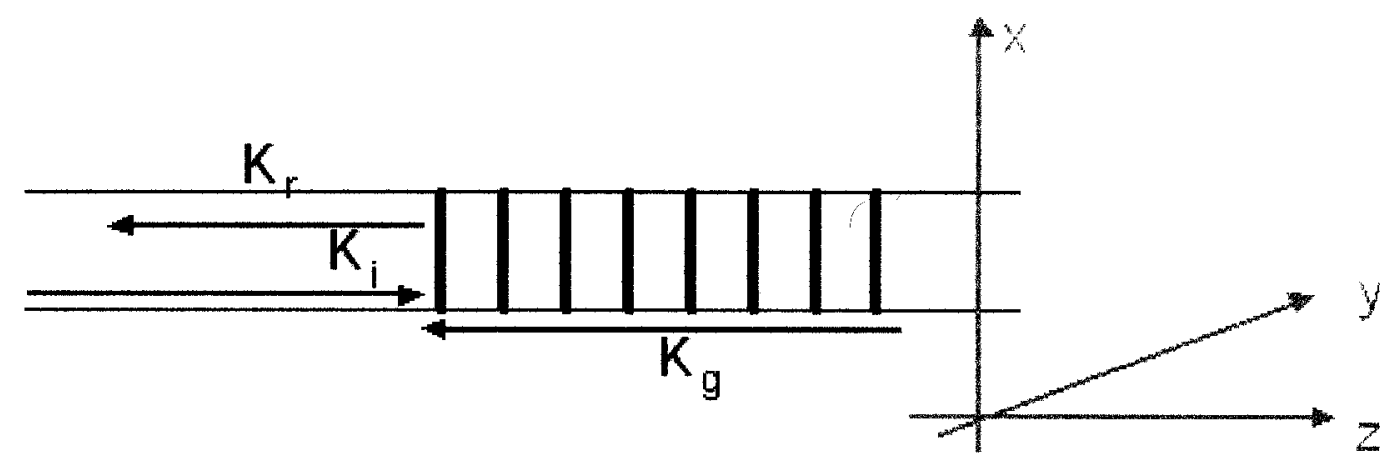

Figure 2.3. Vector diagram of light scattering in the Bragg grating.

For a single mode waveguide momentum propagates along $\mathrm{z}$ with wavevector:

$$
K_{i}=\frac{2 \pi n_{e f f}}{\lambda}
$$

where $\lambda$ is a free space wavelength.

Then the momentum conservation can be written as:

$$
K_{i}+K_{r}=K_{g} \text {, }
$$

Because the waveguide is single mode, reflected back wave must have the same wavevector as the incoming wave (reversed). Assuming that the amplitude of wavevector $K_{\mathrm{g}}$ is $2 \pi / \Lambda$, equation 2.8 changes to:

$$
\frac{2 \pi n_{e f f}}{\lambda}+\frac{2 \pi n_{e f f}}{\lambda}=\frac{2 \pi}{\Lambda}
$$


or

$2 n_{e f f}=\frac{\lambda}{\Lambda}$,

which is the first order Bragg phase matching condition [Othonos and Kalli, 2000].

According to equation (2.9), the phase matching condition can be satisfied for only a couple of modes that propagate in the contra directions and have the same absolute value of the effective index. If the period of the grating matches half the effective wavelength of the propagating core mode, reflected back core mode from a single grating plane will be in the phase with other reflections occurring at other grating planes for the core mode wavelength. Added constructively to each other, reflected fields form a reflection band in the transmission spectrum, which can achieve high reflection efficiency.

Coupling efficiency between contra propagating core modes can be characterized with transverse coupling coefficient $C^{T}$ that can determine the energy transfer between any coupled modes $m$ and $q$ [Othonos and Kalli, 2000] as follows:

$$
C_{q m}^{T}(z)=\frac{\omega}{4} \iint_{\infty} \Delta \varepsilon(x, y, z) e_{q}^{T}(x, y) e_{m}^{T^{*}}(x, y) d x d y
$$

where $\Delta \varepsilon(x, y, z)$ is the permittivity perturbation, $e_{q}^{T}(x, y)$ and $e_{m}^{T}(x, y)$ are transverse mode fields for modes $q$ and $m$. Assuming that the induced index change, $\delta n(x, y, z)$, is uniform across the fiber core and nonexistent outside the core, then the perturbation of the core refractive index can be described as:

$$
\delta n_{c o}(z)=\delta \bar{n}_{c o}(z)\left\{1+s \cos \left[\frac{2 \pi}{\Lambda} z+\phi(z)\right]\right\},
$$

where $\delta \bar{n}_{c o}$ is the 'dc' index change spatially averaged over a grating period, $s$ is the fringe visibility of the index change, $\phi(z)$ describes grating chirp. Self- and crosscoupling coefficients can be found as follows:

$$
\begin{aligned}
& \zeta_{q m}(z)=\frac{\omega n_{c o}}{2} \delta \bar{n}_{c o}(z) \iint_{c o r e} e_{q}^{T}(x, y) \cdot e_{m}^{T *}(x, y) d x d y, \\
& k_{q m}(z)=\frac{s}{2} \zeta_{q m}(z),
\end{aligned}
$$


$\zeta_{q m}(z)$ is also known as 'dc'(period averaged) coupling coefficient and $k_{q m}(z)$ as 'ac' coupling coefficient. Then, the general coupling coefficient can be written as:

$C^{r}{ }_{q m}(z)=\zeta_{q m}(z)+2 k_{q m}(z) \cos \left[\frac{2 \pi}{\Lambda} z+\phi(z)\right]$,

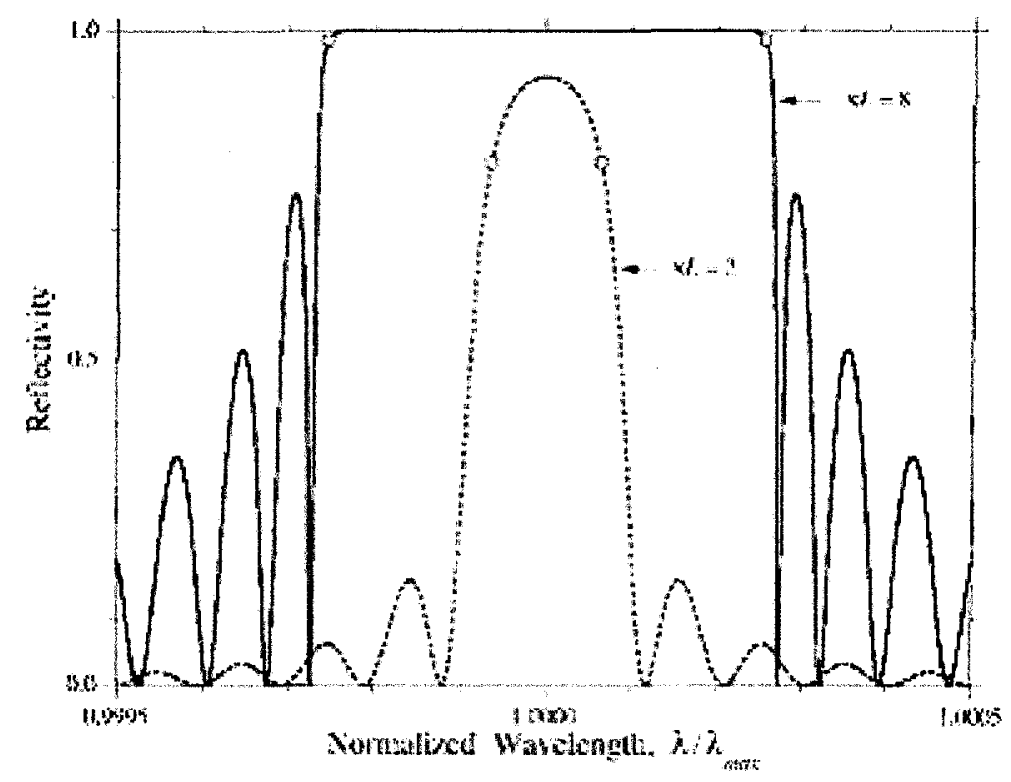

Figure 2.4. Reflection spectra versus normalized wavelength for Bragg reflection in FBG with $k L=2$ and $k L=8$ ( $k$ is the wave number) [Erdogan, 1997].

Figure 2.4 shows reflection spectra for FBGs with different grating lengths. Despite the fact that photoimprinted refractive index modulation $\delta n(x, y, z)$ is not so strong (approximately $10^{-5}$ ), reflection efficiency can achieve $100 \%$ if the Bragg grating has a length of approximately several $\mathrm{mm}$ [Grattan et al., 2000].

Small resonances spaced to the right and left of the Bragg resonance in the spectra are also known as 'sidelobes' and are related to the length of the grating that may not be long enough. Usually sidelobes are recognized as a negative effect, especially for telecommunication applications. For example, a dense wavelength division multiplexing where sidelobes limit interchannel spectral spacing [Malo et al., 1995]. As shown by Matsuhara and Hill, (1974) and Hill, (1974), side lobes can be suppressed by apodization. Apodization can be achieved by photoimprinting the grating that has index modulation amplitude varying across the grating length. 
The bandwidth of the FBG peak is another important parameter. It can be as small as $0.1 \mathrm{~nm}$ (or $12 \mathrm{GHz}$ ) [Berger et al., 2007]. Narrow bandwidths can be achieved in gratings with long grating lengths and weak index modulation. There are several applications of gratings with narrow bandwidths in the telecommunication field. For instance, they can be used as a temporal differentiator for all-optical circuits that allows real-time derivation of arbitrary signals in the optical domain [Berger et al., 2007] or as spectral filters for dense wavelength division-multiplexing transmission systems [Komukai and Nakazawa, 1998].

\subsubsection{Sensing mechanism of FBG}

Most of the sensing applications of FBGs arise not from such characteristics as bandwidth and reflectivity, as it happens in the communications field, but from the relative position of the Bragg wavelength and its relative shift due to changes of external parameters such as temperature, strain and pressure.

The period of the Bragg grating $\Lambda$, which determines the Bragg wavelength, can be affected by thermal expansion of the silicon dioxide material, or by changing the fiber geometry due to strain and/or bending. Therefore, FBG sensors operate by tracking the relative shift of the wavelength of the deep resonance in the transmission spectrum related to the back-reflected light.

Changes of the Bragg peak position dependent on temperature and strain parameters can be found as [Othonos and Kalli, 2000]:

$$
\Delta \lambda_{B}=2\left(\Lambda \frac{\partial n_{e f f}}{\partial l}+n_{e f f} \frac{\partial \Lambda}{\partial l}\right) \Delta l+2\left(\Lambda \frac{\partial n_{e f f}}{\partial T}+n_{e f f} \frac{\partial \Lambda}{\partial T}\right) \Delta T,
$$

where $l$ is the length of the grating, $T$ is the temperature.

The first term of 2.16 relates to strain-induced changes in the optical fiber and can be rewritten as:

$\Delta \lambda_{B}=\lambda_{B}\left(1-p_{e}\right) \varepsilon_{z}$

where $p_{e}$ is an effective strain-optic constant:

$p_{e}=\frac{n_{e f f}^{2}}{2}\left[p_{12}-v\left(p_{11}+p_{12}\right)\right]$, 
and $p_{12}, p_{11}$ are components of the strain-optic tensor, $v$ is the Poisson's ratio [Othonos and Kalli, 2000].

The second term of (2.16) describes changes related to the temperature shift and can be presented as:

$$
\Delta \lambda_{B}=\lambda_{B}\left(\alpha_{\Lambda}+\alpha_{n}\right) \Delta T,
$$

Where $\alpha_{\Lambda}$ is the thermal expansion coefficients and $\alpha_{n}$ thermo-optical coefficients for the fiber.

Despite the fact that Bragg wavelength can be affected by temperature and strain, existing FBG based sensors are not limited to just monitor temperature [Lin et al., 1998] and strain parameters [Rice et al., 2003]. Today it is possible to use FBG fiber based sensors to measure such parameters as directional bending [Martinez et al., 2005], hydrogen concentration [Buric et al., 2007] and material transition dynamics [Cusano et al., 2004]. Most of these sensors utilize the grating sensitivity to temperature and strain, consequently transferring the change of sensed parameter into the temperature- and strain-induced change by using functional coatings or modified fibers.

Chemical sensing is also possible with FBG in modified fibers. In standard unmodified fiber the change of the refractive index of exterior medium will not affect the behavior of FBG (it can not lead to strain or temperature induced change). However, if the cladding of the fiber is reduced, removed completely, or is porous and the field of the back-reflected core mode has the possibility to propagate outside the fiber's cladding the propagation constant of this mode becomes dependent partially on the refractive index of the exterior. As long as the refractive index of the exterior does not exceed the refractive index of the guiding material (cladding or core) and the mode does not become radiative, it is possible to track the change of refractive index of the exterior by means of monitoring the position of the Bragg reflection peak.

Despite the fact that some of the FBG chemical sensors achieve relatively high sensitivity $\left(10^{-5}\right.$ refractive index resolution) [Iadicicco et al., 2005], there are several drawbacks of using FBG technology.

First of all, usually the high sensitivity appears as result of reducing of the cladding diameter. Chemical etching of standard optical fiber or use of the non-standard fibers (for example D-fiber [Meltz et al., 1996]), makes the sensor design more 
complicated and the sensor itself is not so robust as it would be in the case of unmodified standard optical fiber.

Another important limitation of the proposed and implemented FBG chemical sensors is their cross-sensitivity to strain and temperature. Usually, the sensing mechanism requires a secondary referencing grating to eliminate the strain-temperature effect from the experimental readings [Meltz et al., 1991]. Furthermore long lengths of the gratings are required and nonlinear sensitivity within the sensitivity region are additional problems associated with FBG chemical sensors. However, most of these limitations can be overcome by using TFBG technology.

\subsection{Tilted Fiber Bragg Grating (TFBG)}

The performance of FBG can be significantly influenced by introducing a small tilt angle $\vartheta$ in the position of the fringes of the grating relative to the fibers transverse axis $\mathrm{x}$ (Figure 2.5). The addition of a tilt angle results in the coupling of the forward propagating core mode to the back-propagating core mode, and to the set of cladding (bound modes) and possibility to the continuum of radiating modes (for larger tilt angles).

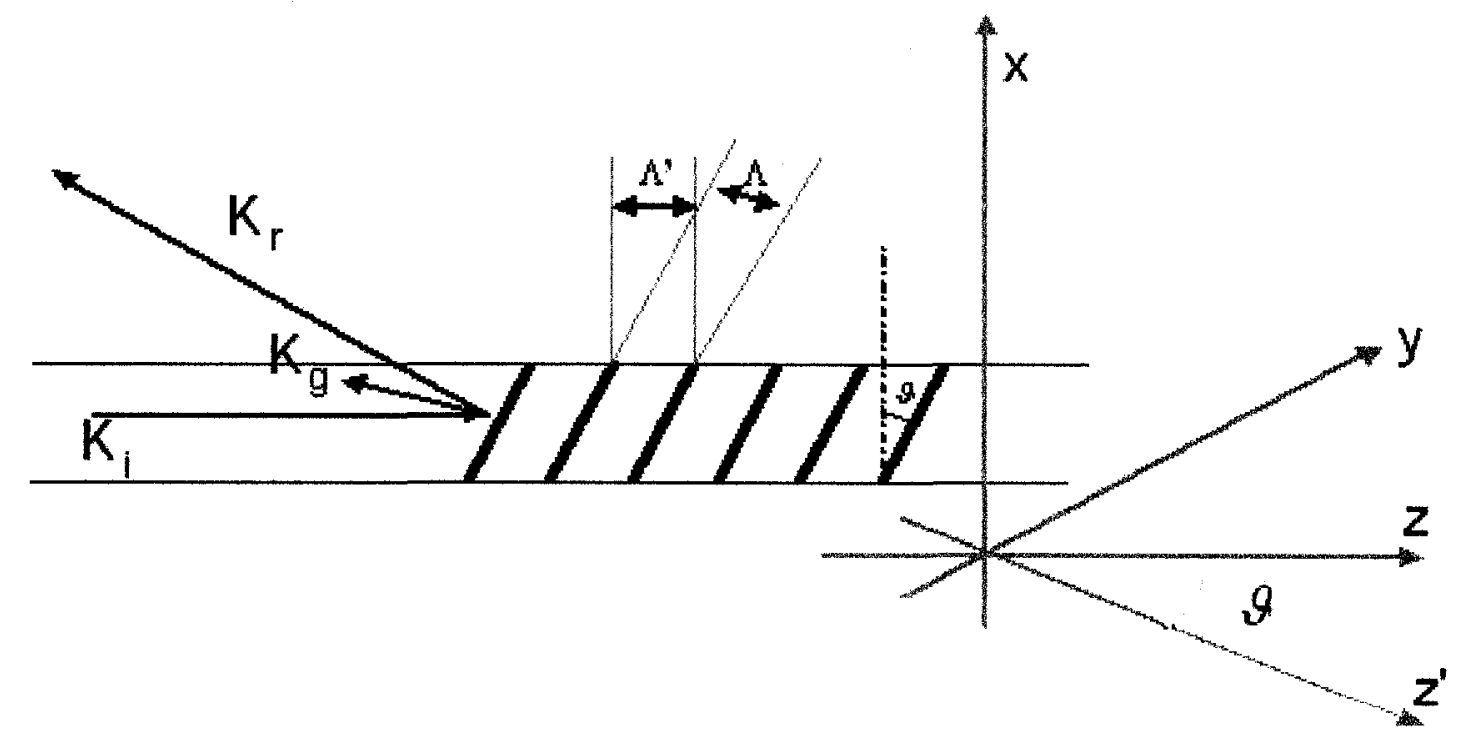

Figure 2.5. Vector diagram of light diffraction in the Tilted Fiber Bragg Grating. 
The Bragg matching condition can be rewritten for the case of a tilted grating by projecting the wavevector of the tilted grating on the axis of propagation as:

$n_{\text {eff }}^{q}+n_{\text {eff }}{ }^{m}=\frac{\lambda^{m}}{\Lambda /(\cos \vartheta)}$,

where $n_{e f f}{ }^{q}$ and $n_{\text {eff }}{ }^{m}$ are the effective indices of the modes to be coupled at wavelength $\lambda^{m}$.

Synonymous to the case for the FBG with no tilt, the matching condition (2.20) can be satisfied for the case when co-propagating core mode $n_{\text {core }}$ couples to counterpropagating core mode $n_{\text {core }}$. However, this condition also can be satisfied for the case when co-propagating core mode $n_{\text {core }}$ couples to the set of cladding modes propagating in backward direction $n_{\text {clad }}{ }^{i}$. The phase matching condition for TFBG can be rewritten as:

$$
n_{\text {core }}+n_{\text {clad }}{ }^{i}=\frac{\lambda^{B}}{\Lambda /(\cos \vartheta)},
$$

The tilt angle $\vartheta$ rotates the induced core refractive index perturbation in such way that (2.12) can be modified to [Erdogan 1997]:

$$
\delta n_{c o}(x, y, z)=\delta \bar{n}_{c o}\left(z^{\prime}\right)\left\{1+s \cos \left[\frac{2 \pi}{\Lambda} z^{\prime}+\phi\left(z^{\prime}\right)\right]\right\} \text {, }
$$

where $z$ ' is the axis shown in Figure 2.5.

Assuming that $z^{\prime} \cong z \cos (\vartheta)$ the general coupling coefficient becomes:

$$
C^{T}{ }_{\mp \pm}(z)=\zeta_{q m}(z)+2 k_{\mp \pm}(z) \cos \left[\frac{2 \pi}{\Lambda} z+\phi(z \cos (\vartheta))\right],
$$

where $m$ and $q$ modes are associated with forward propagating $(+)$ and backward propagating (-) modes. Self- and cross-coupling coefficients in this case are:

$$
\begin{aligned}
& \zeta(z)=\frac{\omega n_{c o}}{2} \delta \bar{n}_{c o}(z \cos (\vartheta)) \iint_{c o r e} e_{\mp}^{T}(x, y) e_{ \pm}^{T *^{*}}(x, y) d x d y, \\
& k_{\mp \pm}(z, \vartheta)=\frac{s}{2} \frac{\omega n_{c o}}{2} \delta \bar{n}_{c o}(z \cos (\vartheta)) \iint_{c o r e} \exp \left( \pm i \frac{2 \pi}{\Lambda} x \tan (\vartheta)\right) e_{\mp}^{T}(x, y) e_{ \pm}^{T *^{*}}(x, y) d x d y,
\end{aligned}
$$


The effect of the introducing of the tilt angle can be expressed through the 'effective fringe visibility' $s_{\mp \pm}(\vartheta) / s$ :

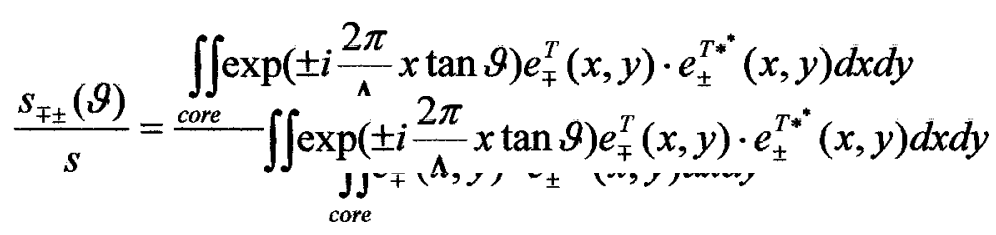

Therefore,

$k_{\mp \pm}(z, \vartheta)=\frac{s_{\mp \pm}(\vartheta)}{2} \varsigma(z)$,

It can be concluded from equations 2.26 and 2.27 that coupling of the copropagating core mode to the counter-propagating core mode is at its maximum if the tilt angle $\vartheta$ is $0^{\circ}$ when the 'effective fringe visibility' reaches it's maximum value. The addition of a tilt angle $\vartheta$ results in a reduction of the fringe visibility for the core mode by a factor expressed in 2.25 and coupling, from the core mode to the core mode, is no longer the strongest in comparing to the coupling to cladding modes.

Figure 2.6 shows an experimental transmission spectrum for a TFBG with 6 degrees of tilt angle. Core resonance along with multiple cladding resonances appear in the spectrum. The figure indicates that the strongest resonance occurred for the backpropagating cladding mode at $1533 \mathrm{~nm}$. Adjusting the tilt angle can easily change the wavelength of the strongest resonance along with the number of cladding resonances [Chen et al, 2007]. This feature of TFBG is actively being used in sensing applications that are going to be discussed in the next chapter. 


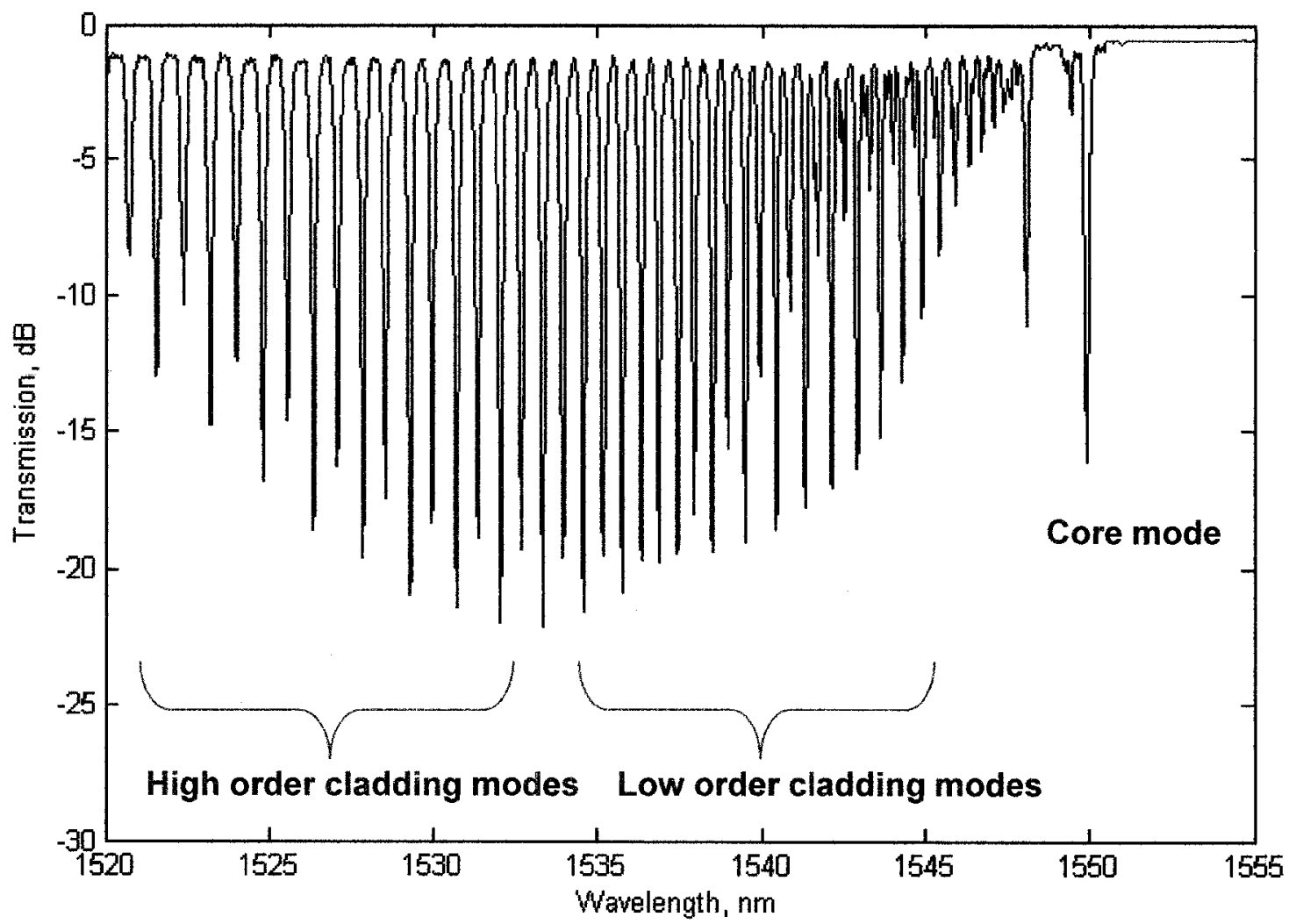

Figure 2.6. Measured transmission spectrum for TFBG with $\vartheta=6^{\circ}$.

\subsubsection{Sensing mechanism of TFBG}

A TFBG has strain and temperature sensitivity similar to a FBG, however, the propagation of back reflected cladding modes in proximity to cladding-exterior medium interface makes the propagation constant of these modes dependent on the refractive index of the exterior. Equation 2.16 describing the Bragg wavelength shift for FBGs can be rewritten for TFBG back-reflected core and cladding modes [Chen and Albert, 2006; Chen et al., 2007]:

$$
\begin{aligned}
& \Delta \lambda_{B}=2\left(\frac{\Lambda}{\cos (\vartheta)} \frac{\partial n_{e f f}}{\partial T}+\frac{n_{e f f}}{\cos (\vartheta)} \frac{\partial \Lambda}{\partial T}\right) \Delta T+2\left(\frac{\Lambda}{\cos (\vartheta)} \frac{\partial n_{e f f}}{\partial l}+\frac{n_{e f f}}{\cos (\vartheta)} \frac{\partial \Lambda}{\partial l}\right) \Delta l+ \\
& +2\left(\frac{\Lambda}{\cos (\vartheta)} \frac{\partial n_{e f f}}{\partial n_{e x t}}+\frac{n_{e f f}}{\cos (\vartheta)} \frac{\partial \Lambda}{\partial n_{e x t}}\right) \Delta n_{e x t}
\end{aligned}
$$




$$
\begin{aligned}
& \Delta \lambda_{\text {clad }}=\left(\frac{\Lambda}{\cos (\vartheta)} \frac{\partial\left(n_{\text {eff }, \text { core }}+n_{\text {eff }, \text { clad }}\right)}{\partial T}+\frac{n_{\text {eff,core }}+n_{\text {eff,clad }}}{\cos (\vartheta)} \frac{\partial \Lambda}{\partial T}\right) \Delta T+ \\
& \left(\frac{\Lambda}{\cos (\vartheta)} \frac{\partial\left(n_{\text {eff,core }}+n_{\text {eff,clad }}\right)}{\partial l}+\frac{n_{\text {eff,core }}+n_{\text {eff,clad }}}{\cos (\vartheta)} \frac{\partial \Lambda}{\partial l}\right) \Delta l+ \\
& \left(\frac{\Lambda}{\cos (\vartheta)} \frac{\partial\left(n_{\text {eff }, \text { core }}+n_{\text {eff,clad }}\right)}{\partial n_{\text {ext }}}+\frac{n_{\text {eff,core }}+n_{\text {eff }, \text { clad }}}{\cos (\vartheta)} \frac{\partial \Lambda}{\partial n_{\text {ext }}}\right) \Delta n_{\text {ext }}
\end{aligned}
$$

Temperature-induced shifts of the core mode resonance $\Delta \lambda_{B}$ and cladding mode resonances $\Delta \lambda_{\text {clad }}^{i}$ have very close values [Jafari, 2006]. Chen et al, (2007). showed that temperature-induced shifts for cladding modes relative to the core mode shift are less than $\pm 12 \mathrm{pm}$ within the temperature range from $-10^{\circ} \mathrm{C}$ to $70^{\circ} \mathrm{C}$. By monitoring the wavelength difference between the core mode resonance and one of the cladding mode resonances, it is possible to eliminate temperature effects from the sensor's readings. This provides a great advantage for the TFBG sensor technology allowing the creation of a temperature-independent strain or chemical sensor.

Strain-related parts of (2.28) and (2.29) can be rewritten as:

$$
\begin{aligned}
& \Delta \lambda_{B}=\lambda_{B}\left(1-p_{B}\right) \Delta \varepsilon, \\
& \Delta \lambda_{\text {clad }}^{i}=\lambda_{\text {clad }}^{i}\left(1-p_{\text {clad }}^{i}\right) \Delta \varepsilon,
\end{aligned}
$$

where $p_{B}$ and $p_{\text {clad }}^{i}$ are photoelastic coefficients and can be found as:

$$
\begin{aligned}
p_{B} & =-\frac{1}{n_{e f f}} \frac{\partial n_{e f f}}{\partial \varepsilon}, \\
p_{\text {clad }}^{i} & =-\frac{1}{n_{e f f, c o r e}^{i}+n_{e f f, c l a d}^{i}} \frac{\partial\left(n_{e f f, c o r e}^{i}+n_{e f f, c l a d}^{i}\right)}{\partial \varepsilon},
\end{aligned}
$$

The effective refractive index for higher order modes has a smaller value than the low order modes and therefore results in a smaller strain-related variation in the spectrum for high-order modes.

Now the change of the exterior refractive index should not affect the period of the grating and the core mode effective index: $\frac{\partial n_{e f f}}{\partial n_{e x t}} \approx 0$ and $\frac{\partial \Lambda}{\partial n_{e x t}} \approx 0$. Hence the Bragg wavelength is independent of the exterior refractive index variation $\Delta n_{\text {ext }}$ and the third statement of equation 2.28 can be rewritten as: 
$\Delta \lambda_{B}=0$,

However, cladding modes are still dependent on $\Delta n_{\text {ext }}$ :

$\Delta \lambda_{\text {clad }}=\frac{\Lambda}{\cos (\grave{\vartheta})} \frac{\partial n_{\text {eff }, \text { clad }}}{\partial n_{\text {ext }}} \Delta n_{\text {ext }}$,

These effects were experimentally verified by Chen and Albert, (2006) and Chen et al, (2007).

Because of the sensitivity to $n_{\text {ext }}$ the TFBG enables fabrication a high-quality chemical sensor, which has several advantages over other possible chemical sensors based on FBG or Long-Period Gratings (LPG). Presence of the cladding mode resonances in the spectrum, approximately 50 overall, makes the TFBG able to monitor a refractive index change within a wide range (1-1.43 $\left.n_{\text {ext }}\right)$. For example, TFBG with $6^{\circ}$ of tilt angle is able to track refractive index changes within the 1.377-1.43 range; however, this operating range can be extended towards lower refractive indices by increasing the tilt angle.

For comparison, LPGs use only one or two cladding mode resonances to track the refractive index change and to achieve the sensitivity over the same refractive index range several $L P G$ or $L P G$ and FBG should be incorporated together in a single device.

The sensitivity of the TFBG sensor can achieve $10000 \mathrm{pm} / \mathrm{u} . \mathrm{r} . \mathrm{i}$; assuming the minimal spectral resolution is less than $1 \mathrm{pm}$, it is possible to distinguish refractive index change as small as $10^{-4}$ [Chen et al., 2007]. 


\section{Chapter 3. Surface Plasmon Resonance}

\section{Objectives:}

The objectives of this chapter are to provide an introduction of the Surface Plasmon Resonance effect and available SPR techniques. The first section includes information about SPR theory. It shows the derivation of the phase-matching condition that should be satisfied in order to excite SPR and determine SPR characteristics. The second section describes the main techniques that are currently employed in the SPR field such as prism and fiber configurations. A literature review regarding available SPR fiberbased sensors is presented in Subsection 3.2.2.

\subsection{Surface Plasmon Resonance Theory}

The SPR effect can be explained through the definition of a Surface Plasmon Wave (SPW). SPW, also known as Surface Plasmon-Polariton (SPP), is a non-radiative, surface electromagnetic wave, which propagates at the boundary between mediums having dielectric constants of the opposite sign [Welford, 1988]. Usually, one medium is dielectric while the other is metal or doped high-mobility semiconductors such as InSb [Tilley, 1988]. Metals and doped high-mobility semiconductors, otherwise known as surface-active mediums, are characterized by the presence of a free electron gas in the material matrix, which behaves almost as free electron plasmas [Ligler and Taitt, 2002]. When photons or electrons excite the electron plasma, it coherently oscillates in the direction normal to the interface between materials and results in propagation of the transverse magnetic waves at this interface [Raether, 1988]. Since the SPW is an electromagnetic wave, such important parameters as propagation constant, length of propagation and depth of penetration in both mediums, with conditions for exciting SPR, are found using Maxwell's equations.

In the subsequent paragraphs of Section 3.1, Maxwell's equations are used to derive a formulation of the Transverse Electric (TE) and Transverse Magnetic (TM) modes by applying a slab waveguide model, which simplifies the three-dimension problem of localization of the electromagnetic field to the two-dimension problem. Expressions for TM and TE modes paired with boundary conditions are used to find the dispersion equation and exact field characteristics for specific waveguide cases. In 
addition, boundary conditions are introduced for the surface mode therefore making it possible to derive SPR dispersion relations for metal-dielectric and dielectric-metaldielectric interfaces. The boundary condition which is used in the derivation of the dispersion equation is such that in order for the surface wave to be physically possible, all the electromagnetic fields must decay to zero at $x=+/-\infty$.

\subsubsection{Electromagnetic field propagation in the slab waveguide}

Initially, the propagation of an electromagnetic field in the slab waveguide displayed in Figure 3.1 is considered.

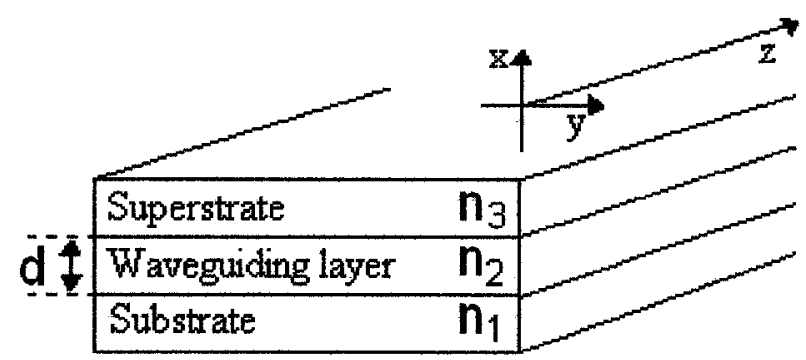

Figure 3.1. Planar waveguide with step refractive index profile.

The source-free Maxwell's equations in terms of electrical and magnetic field vectors $\vec{E}(r, t)$ and $\vec{H}(r, t)$ [Homola, 2006] are:

$$
\begin{aligned}
& \nabla \times \vec{E}(r, t)+\mu \frac{\partial \vec{H}(r, t)}{\partial t}=0, \\
& \nabla \cdot(\mu \vec{H}(r, t))=0, \\
& \nabla \times \vec{H}(r, t)-\varepsilon_{0} \varepsilon(r) \frac{\partial \vec{E}(r, t)}{\partial t}=0, \\
& \nabla \cdot\left(\varepsilon_{0} \varepsilon(r) \vec{E}(r, t)\right)=0,
\end{aligned}
$$

where $r$ is a space vector, $t$ is time, $\mu$ is magnetic permeability of the medium ${ }^{1}, \varepsilon$ is the relative permittivity of the medium ${ }^{2}$.

\footnotetext{
${ }^{1}$ for non-magnetic materials magnetic permeability is equal to free space magnetic permeability:

$\mu=\mu_{0}$,

where $\mu_{0}=4 \pi \cdot 10^{-7}[H / m]$
} 
If modal fields can propagate inside the waveguide can be determined as:

$$
\begin{aligned}
& \vec{E}=E(x, y) \exp (i(\beta z-\omega t) \\
& \vec{H}=H(x, y) \exp (i(\beta z-\omega t),
\end{aligned}
$$

where $\omega$ is an angular frequency, $\beta$ is the propagation constant, then equations 3.1 to 3.4 can be rewritten as:

$$
\begin{aligned}
& \left\{\begin{array}{l}
\frac{\partial E_{z}}{\partial y}+j \beta E_{y}=-j \omega \mu_{0} H_{x}, \\
-j \beta E_{x}-\frac{\partial E_{z}}{\partial x}=-j \omega \mu_{0} H_{y} \\
\frac{\partial E_{y}}{\partial x}-\frac{\partial E_{x}}{\partial y}=-j \omega \mu_{0} H_{z}
\end{array}\right. \\
& \left\{\begin{array}{l}
\frac{\partial H_{z}}{\partial y}+j \beta H_{y}=j \omega \varepsilon_{0} n^{2} E_{x} \\
-j \beta H_{x}-\frac{\partial H_{z}}{\partial x}=j \omega \varepsilon_{0} n^{2} E_{y} \\
\frac{\partial H_{y}}{\partial x}-\frac{\partial H_{x}}{\partial y}=j \omega \varepsilon_{0} n^{2} E_{z}
\end{array}\right.
\end{aligned}
$$

The groups of equations 3.7 and 3.8 can be used to describe the field propagation in waveguides with any refractive index profile. By applying a three-layer optical waveguide model (Figure 3.2) it is assumed that electromagnetic fields $E$ and $H$ do not have $y$-axis dependency resulting in a simplification of the field localization to a twodimensional problem. Substituting of $\frac{\partial E}{\partial y}=0$ and $\frac{\partial H}{\partial y}=0$ in the equations 3.7 and 3.8 allows the field's solution to be found in the form of transverse modes: TE (3.9) and TM (3.10):

${ }^{2}$ permittivity $\varepsilon_{\chi}$ of material can be found as:

$\varepsilon_{\chi}=\varepsilon_{0} \varepsilon$,

where $\varepsilon_{0}$ is the permittivity of the vacuum [Okamoto, 2000] and $\varepsilon$ is the relative permittivity for non-magnetic materials $\left(\varepsilon=n^{2}\right)$. 


$$
\begin{aligned}
& \frac{\partial^{2} E_{y}}{\partial x^{2}}+\left(k^{2} n^{2}-\beta^{2}\right) E_{y}=0, \\
& H_{x}=-\frac{\beta}{\omega \mu_{0}} E_{y}, \\
& H_{z}=\frac{j}{\omega \mu_{0}} \frac{\partial E_{y}}{\partial x}, \\
& E_{x}=E_{z}=H_{y}=0, \\
& \frac{1}{n^{2}} \frac{\partial^{2} H_{y}}{\partial x^{2}}+\left(k^{2}-\frac{\beta^{2}}{n^{2}}\right) H_{y}=0, \\
& E_{x}=-\frac{\beta}{\omega \varepsilon_{0} n^{2}} H_{y}, \\
& E_{z}=-\frac{j}{\omega \varepsilon_{0} n^{2}} \frac{\partial H_{y}}{\partial x}, \\
& H_{x}=H_{z}=E_{y}=0,
\end{aligned}
$$

As it can be noted from the group of Equations in 3.9 and 3.10, TE and TM modes are characterized respectively by electric and magnetic fields situated in the $x-y$ plane that is perpendicular to the $\mathrm{z}$-axis.

\subsubsection{Field distribution in the slab waveguide}

Solving the equations for TE and TM modes while applying boundary conditions leads to the formulation of the dispersion equation (or eigenvalue equation) where the propagation constant and field's distribution can be achieved. Boundary conditions for the case of the slab waveguide with step-refractive index profile are:

1) propagating in the optical slab waveguide with $n 2>n 1$ and $n 2>n 3$ light is confined in the core and exponentially decays in the cladding; and

2) transverse field components ( $E_{y}, H_{z}$ for TE mode; $\mathrm{H}_{\mathrm{y}}, \mathrm{E}_{\mathrm{z}}$ for TM mode) should be continuous at the interfaces between waveguiding layer, substrate and superstrate [Okamoto 2000].

\subsubsection{SPW at the interface between semi-infinite mediums}

Plasmon wave characteristics can be found by applying a waveguide model similar to what was previously used, but with a waveguide layer approaching zero 
$(d \rightarrow 0)$ (Figure 3.1). Let's assume that both superstrate and substrate are made of materials which dielectric functions are complex functions of frequency as follows:

$\varepsilon_{1}(\omega)=\varepsilon_{1}^{\prime}(\omega)+i \varepsilon_{1}(\omega)$, dielectric function of the first material

$\varepsilon_{2}(\omega)=\varepsilon_{2}^{\prime}(\omega)+i \varepsilon_{2}{ }_{2}(\omega)$, dielectric function of the second material

Taking into account that the SPW should be localized at the interface and must decay to zero at $x=+/-\infty$, only TM modes (Equation 3.10) can satisfy these requirements [Tilley, $1988]$.

Assuming that a TM mode propagates at the interface along axis $z, E$ field can defined as:

$E=\left(E_{1 x}, 0, E_{1 z}\right) \exp (i(\beta z-\omega t)) \exp \left(i k_{1} x\right)$ in the medium one,

$E=\left(E_{2 x}, 0, E_{2 z}\right) \exp (i(\beta z-\omega t)) \exp \left(i k_{2} x\right)$ in the medium two,

where $E_{i x}, E_{i z}$ are projections of the $E$ field's amplitude on the axes in the $\mathrm{i}^{\text {th }}$ medium, $k_{i}$ is the projection of the wave vector on the $\mathrm{x}$-axis in the $\mathrm{i}^{\text {th }}$ medium.

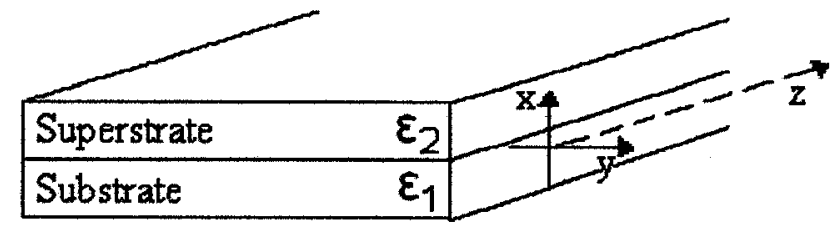

Figure 3.2. Interface between semi-infinite mediums with permittivity $\varepsilon_{1}$ and $\varepsilon_{2}$.

The dispersion relation for SPW is obtained by considering boundary condition one, which states that at the surface $x=0$, the surface mode's tangential component should be continuous:

$E_{1 z}=E_{2 z}$,

And the second boundary condition where the depth of penetration of SPW in both mediums is finite and can be found based on the fact that $\vec{D}$ (electrical displacement $\vec{D}=\varepsilon(\omega) \vec{E})$ is the same in both mediums:

$\varepsilon_{1} E_{1 x}=\varepsilon_{2} E_{2 x}$,

Condition $\nabla . D=0$ helps to obtain the ratio between orthogonal components of $E$ : 
$\beta E_{i z}+k_{i} E_{i x}=0$

These 3 conditions 3.15 to 3.17 lead to the next dispersion relations:

$$
\begin{aligned}
& \frac{k_{1}}{\varepsilon_{1}}=\frac{k_{2}}{\varepsilon_{2}}, \\
& \beta^{2}=\frac{\omega^{2}}{c^{2}} \frac{\varepsilon_{2} \varepsilon_{1}}{\varepsilon_{2}+\varepsilon_{1}},
\end{aligned}
$$

where $c$ is the speed of the light in a vacuum.

Equation 3.18, since $k_{1}$ and $k_{2}$ have opposite signs, for SPW to exist $\varepsilon_{1}$ and $\varepsilon_{2}$ should also have opposite signs. Given that, Equation 3.19 can have a positive $\beta$ only if:

$\varepsilon_{1}+\varepsilon_{2}<0$

\subsubsection{Characteristics of SPW}

In order for the SPW wave to be physically possible, mediums should have opposite signs of permittivity and negative permittivity should have a bigger absolute value than the positive permittivity. This requirement can be satisfied if one of the mediums is dielectric (which usually has positive permittivity) and another is metal. "Noble" metals, such as gold and silver are the most frequently used and highly doped semiconductors are rarely used as mediums with negative permittivity. Free electron gas presented in these materials reflects an external electromagnetic field if the applied frequency $\omega$ is less than the plasma frequency of these materials $\omega_{p}$ :

$$
\omega_{p}=\sqrt{\frac{N e^{2}}{\varepsilon_{0} m_{e}}},
$$

where $\mathrm{N}$ is the concentration of free electrons, e and $m_{e}$ are the electron charge and mass respectively. Negative permittivity occurs when the applied wavelengths are bigger than the plasma wavelength and the plasma becomes non-transparent. Such metals as silver, gold and aluminum have a negative real part of permittivity for visible and infrared wavelengths and their plasma wavelength lies in the ultraviolet region [Homola, 2006]. The plasma wavelength for semiconductors is located in the infrared region [Blakemore, $1985]$. 
Assuming that one of the mediums involved in the excitation of the SPR is a metal $\left(\varepsilon_{\mathrm{m}}\right)$ and another one is a dielectric $\left(\varepsilon_{\mathrm{d}}\right)$, further notations of medium one $\left(\varepsilon_{1}\right)$ and medium two $\left(\varepsilon_{2}\right)$ will be replaced by $\varepsilon_{\mathrm{m}}$ and $\varepsilon_{\mathrm{d}}$.

The complex metal's permittivity results in the SPW's complex propagation constant:

$$
\beta=\beta^{\prime}+i \beta^{\prime \prime}=\frac{\omega}{c} \sqrt{\frac{\varepsilon_{m}^{\prime} \varepsilon_{d}}{\varepsilon_{m}^{\prime}+\varepsilon_{d}}}+i \frac{\varepsilon_{m}^{\prime \prime}}{2\left(\varepsilon_{m}^{\prime}\right)^{2}} \frac{\omega}{c}\left(\frac{\varepsilon_{m}^{\prime} \varepsilon_{d}}{\varepsilon_{m}^{\prime}+\varepsilon_{d}}\right)^{3 / 2},
$$

The term $\beta^{\prime}$ describes the real part of the propagation constant relating to the effective refractive index $N_{\text {eff }}$ [Ligler and Taitt, 2002]:

$$
N_{e f f}=\frac{c}{\omega} \operatorname{Re}\{\beta\}=\frac{c}{\omega} \beta^{\prime}
$$

But the propagation constant has an imaginary part $\beta "$, which is caused by the imaginary part of the metal's permittivity and which causes attenuation of the plasmon wave's.

The length of attenuation can be found as [Homola, 2006]:

$L=\frac{1}{2 \beta^{\prime \prime}}$

Equations $3.13,3.14$ and 3.19 can be used to find the plasmon electrical and magnetic fields distribution:

$$
\begin{aligned}
& H_{y}(x)=A \exp \left(\gamma_{m} x\right), \text { for } x<0 \\
& H_{y}(x)=A \exp \left(-\gamma_{d} x\right), \text { for } x>0 \\
& E_{x}(x)=A \frac{\beta}{\omega \varepsilon_{m} \varepsilon_{0}} \exp \left(\gamma_{m} x\right), \text { for } x<0 \\
& E_{x}(x)=A \frac{\beta}{\omega \varepsilon_{d} \varepsilon_{0}} \exp \left(-\gamma_{d} x\right), \text { for } x>0 \\
& E_{z}(x)=A \frac{\gamma_{m}}{\omega \varepsilon_{m} \varepsilon_{0}} \exp \left(\gamma_{m} x\right), \text { for } x<0 \\
& E_{z}(x)=-A \frac{\gamma_{d}}{\omega \varepsilon_{d} \varepsilon_{0}} \exp \left(-\gamma_{d} x\right), \text { for } x>0
\end{aligned}
$$

where 
$\gamma_{m}=i \frac{\omega}{c} \frac{\varepsilon_{m}}{\sqrt{\varepsilon_{m}+\varepsilon_{d}}}$

$\gamma_{d}=i \frac{\omega}{c} \frac{\varepsilon_{d}}{\sqrt{\varepsilon_{m}+\varepsilon_{d}}}$

and $\mathrm{A}$ is the modal field amplitude.

These functions can be plotted to illustrate the SPW field distribution at the metaldielectric interface. Figure 3.3 shows the magnetic field distribution for SPW at the boundary between gold and dielectric with a refractive index of 1.32 .

As it appears the maximum of the field is at the metal-dielectric interface. Penetration of electromagnetic field inside metal and dielectric $L_{p m}$ and $L_{p d}$ is defined as the distance from the interface at which the amplitude of the field has fallen to 1/e of its value at the surface [Ligler and Taitt, 2002] and can be found as:

$$
\begin{aligned}
& L_{p m}=1 / \operatorname{Re}\left\{\gamma_{m}\right\}, \\
& L_{p d}=1 / \operatorname{Re}\left\{\gamma_{d}\right\},
\end{aligned}
$$

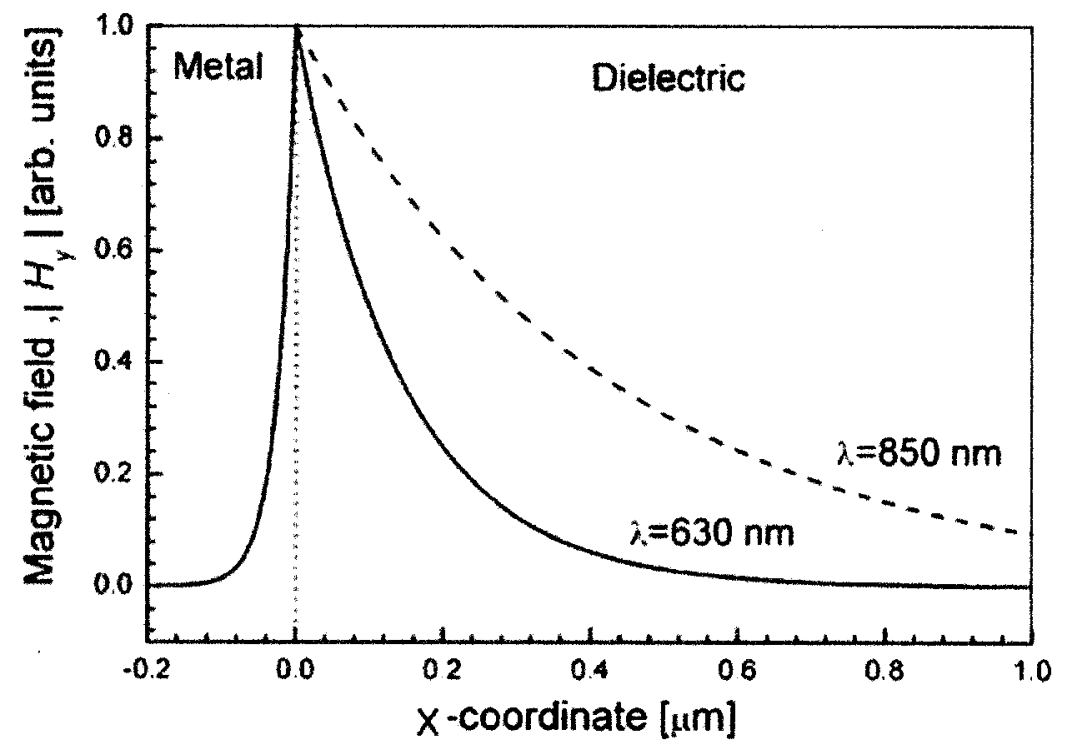

Figure 3.3. Distribution of the magnetic field intensity for an SPW at the interface between gold and dielectric (refractive index of the dielectric 1.32) in the direction perpendicular to the interface calculated for two different wavelengths [Homola, 2003]. 
The penetration depth into the dielectric is bigger than the penetration depth into the metal and can vary from 100 to $500 \mathrm{~nm}$ in the visible and near infrared regions [Ligler and Taitt, 2002]. Such deep penetration (comparing to the penetration into the metal) makes the propagation constant of SPW very sensitive to the changes of the refractive index of the dielectric and makes it achievable to realize chemical and biochemical sensors based on SPR. For bio-chemical sensors, usually the biomolecular recognizing element (ligand) is attached to the gold layer as a means to capture the analyte (target) in the dielectric medium. This results in a change of the refractive index of the biolayer that consequently leads to a change of the propagation constant of the SPR. If the length the target-agent complex is equal to the penetration depth of the SPW into the dielectric then the change of the propagation constant of SPW is directly proportional to the change of the refractive index of the dielectric $\Delta n$ :

$\operatorname{Re}\{\Delta \beta\} \cong k \Delta n$,

If the length of the target-agent complex $d$ is much smaller than the penetration depth of the SPW, the change of the propagation constant of the SPW can be found as:

$\operatorname{Re}\{\Delta \beta\} \cong \frac{2 n_{s} n_{f} k^{2} d}{\sqrt{\left|\operatorname{Re}\left\{\varepsilon_{m}\right\}\right|}} \Delta n$,

where $n_{s}, n_{f}$ are the refractive indices of biolayer and background dielectric medium.

\subsubsection{Propagation of SPW at the dielectric-metal-dielectric interface}

In practice, the SPR is usually observed in the waveguide structure where the metal layer is sandwiched between two layers of dielectric (Figure 3.4) or vice versa, the dielectric layer is sandwiched by two layers of the metal. The situation here is different from the case when SPR is guided at the interface between two semi-infinite materials and a lot of research has involved finding solutions to this problem. This area of research is of great interest because the possibility to use the metal-dielectric waveguide structures for creating passive and active components for highly integrated photonics structures [Boltasseva et al., 2006]. For a more in depth discussion of this issue, the reader can refer to the following [Wendier and Haupt, 1986; Burke et al., 1986; Berini 2000; Berini 2001]. 
It should be noted that if the thickness of the metal layer surrounded by the dielectric is smaller than the penetration depth of SPW in it, SPWs guided at both interfaces are being coupled due to the field tunneling through the metal layer which results in four mixed electromagnetic fields. These electromagnetic fields can have different characteristics that depend not only on the wavelength and dielectric functions of the materials involved, but also on the metal layer thickness, width [Berini, 2001] and waveguide symmetry. If the thickness of the metal layer is big enough (approximately $>100 \mathrm{~nm}$ ), each interface guides SPW independently.

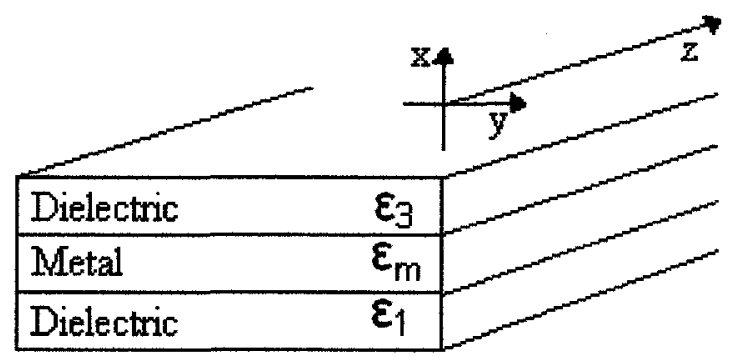

Figure 3.4. Dielectric-metal-dielectric waveguide.

\subsection{SPW excitation by light}

In order to excite SPR by irradiation with light, a matching condition should be satisfied which states that the excitation of SPR is possible only if the propagation constant of the light vector matches the propagation constant of the SPW [Ligler and Taitt, 2002]. According to the plasmon dispersion relation [Raether, 1988] for any wavelength, SPW have a longer wave vector than light waves of the same frequency propagating at the surface.

This condition makes it impossible to excite SPW directly by incident light onto a smooth metal surface. The wavevector of light can be adjusted to match the wavevector of the SPW by launching it through the metal from a medium with a refractive index higher than the refractive index of medium at the boundary where SPW is going to be excited. Standard ways to excite SPR are to couple light through a prism, grating or waveguide [Homola, 2003]. However, in recent years there has been quite an interest to excite SPR on the surface of optical fibers. However, prism coupling is one of the first tested SPR configurations and is going to be discussed in the subsequent section. 


\subsubsection{Prism configuration}

The well-established Kretschmann configuration, which was first described by Kretschmann and Raether in 1968 [Kretschmann and Raether, 1968] was one of the first methods used to excite SPR. This configuration is shown in Figure 3.5 where the setup employs a high refractive index prism with a metal layer on the prism's bottom and a dielectric on the other side of the metal layer. Light propagating in the prism is reflected at the prism-metal layer interface by means of the total internal reflection mechanism. Evanescent field of reflected light at the first interface penetrates into the metal, and if the thickness of the layer allows, it reaches the second metal-dielectric interface. If the refractive index of the second dielectric is smaller than the refractive index of the prism and also, if the propagation constant of light propagating in the prism matches the propagation constant of SPW, then a plasmon resonance occurs and a plasmon wave propagates at the second metal-dielectric interface.

The phase-matching condition that was mentioned in the previous paragraph is:

$$
\sqrt{\varepsilon_{p}} \sin (\theta)=\operatorname{Re}\left\{\sqrt{\frac{\varepsilon_{m} \varepsilon_{d}}{\varepsilon_{m}+\varepsilon_{d}}}\right\}
$$

where $\theta$ is the angle of incidence, $\varepsilon_{p}, \varepsilon_{m}$ and $\varepsilon_{d}$ are the dielectric functions of the prism, metal and dielectric medium $\left(\varepsilon_{p}>\varepsilon_{d}\right)$. 


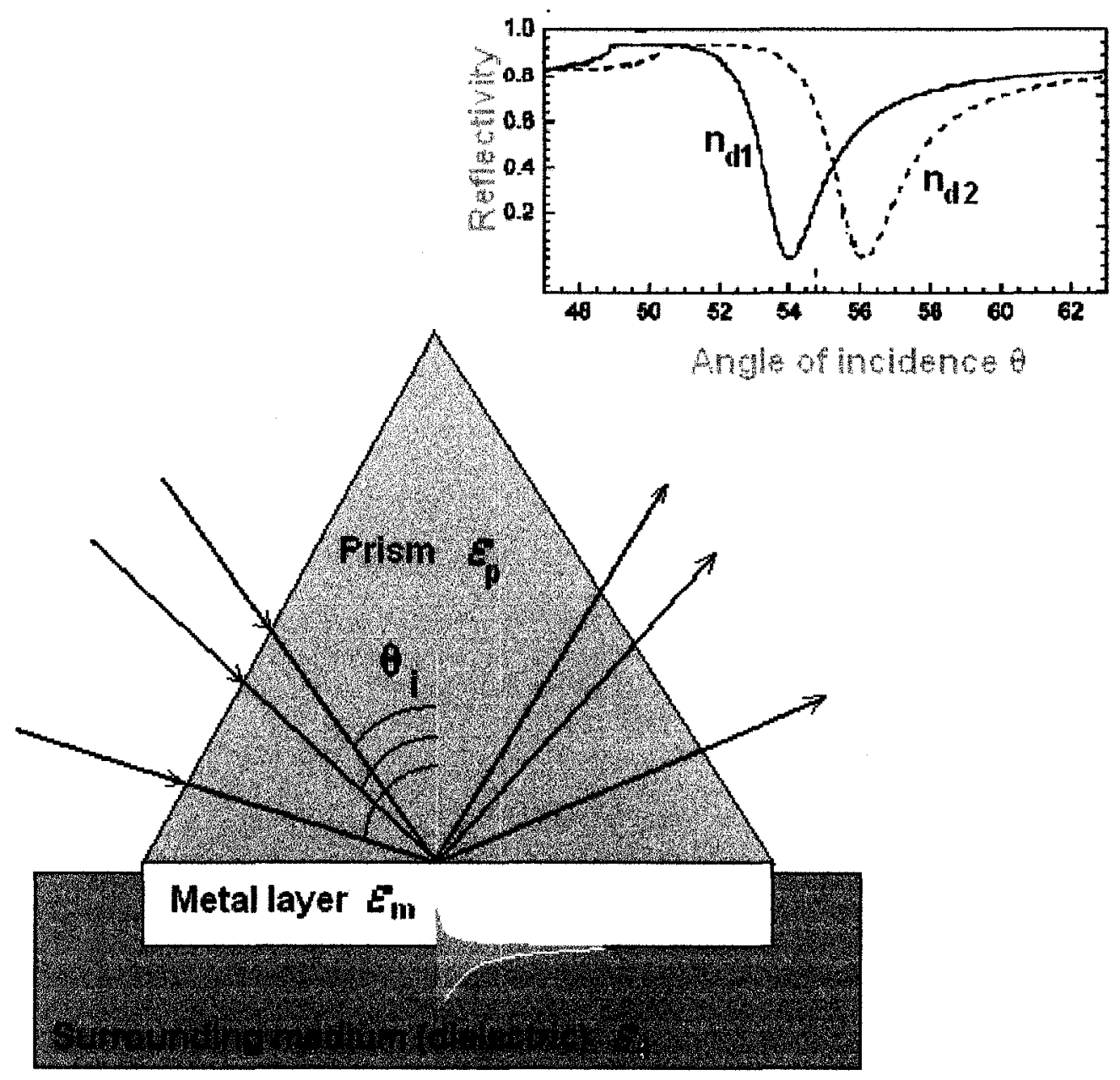

Figure 3.5. Kretschmann configuration.

For specific refractive indices of the surrounding medium (second dielectric $\varepsilon_{d}$ ) and specific wavelengths of light propagating in the prism, there is only one such angle $\theta$ when the matching condition is satisfied and SPR can be excited. By changing the angle $\theta$ at which light propagates in the prism, it is possible to change the parallel component of the light's wave vector, the propagation constant, and to find that the evanescent field at the metal-dielectric layer couples to the SPW.

If coupling occurs and the SPW interacts with the light wave, some of the light wave's parameters such as amplitude, phase, polarization, and spectral distribution can be altered. For instance, in the angle scanning mechanism, which is shown at the Kretschmann configuration, the SPR resonance will result in a strong decrease of the light reflected from the base of the prism. 
The measurement of these parameters (intensity, phase...) makes it possible to track changes of the refractive index of the dielectric on that side of the metal where SPR occurs. According to which parameter is going to be measured, SPR sensors can be classified as angle, wavelength, intensity, phase or a polarization-modulation sensor. For example, in wavelength-modulation based sensors, SPR can be measured by tracking the resonant wavelength at which SPR occurs at a fixed angle of incidence (Figure 3.6).

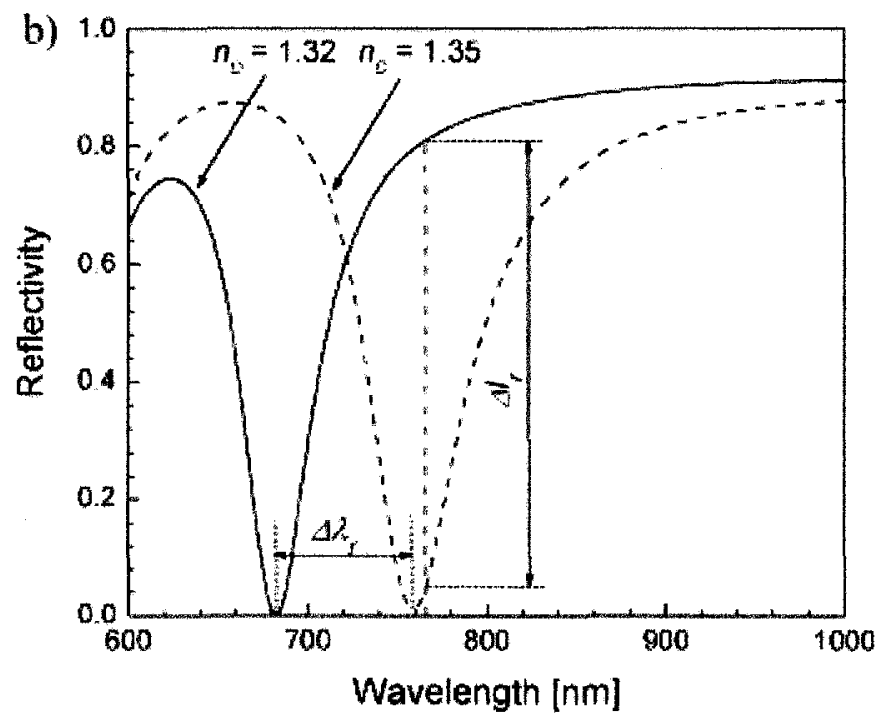

Figure 3.6. Reflectivity for light wave exciting an SPW in the geometry versus wavelength for two different refractive indices of the dielectric (angle of incidence is $54^{\circ}$ ) [Homola, 2003].

The SPR sensor with a prism configuration as a platform for sensing was commercialized by several companies including: Biacore [Baron, 2004], Texas Instruments [Texas Instruments, 2002], Autolab [Autolab, 2007], IBIS [IBIS Technologies, 2006], Reichert [Reichert, 2007] and XanTec [XANTEC Bioanalytics, 2007]. Even though the first commercialized SPR biosensors were developed for laboratory measurements and were rather bulky, today's biosensing platforms based on the Kretschmann configuration can be compact, low cost and portable devices, which can work not just in laboratories, but also in the field [Texas Instruments, 2003]. The reported sensitivity of the prism-based sensors is about $10^{-6}-10^{-7}$ [Wang et al., 2000]. 


\subsubsection{Absorption-based SPR sensing theory}

Absorption-based SPR theory is a "standard" Kretschmann theory, which takes into account the absorption characteristics of all mediums including prism, metal layer and sensed medium [Kurihara and Suzuki, 2002]. It was specifically developed for the prism configuration and it is based, besides Kretschmann's theory, on the three-layer Fresnel equation for reflectance and Lorentz's model for the sensed medium (surrounding medium). Overall, the absorption-based SPR theory is a fairly useful tool that can explain the behavior of resonance SPR curves and their dependence on wavelength. Moreover, it can be used to characterize metal coating thickness and find the optimal thickness to maximize SPR.

The optimal thickness for the metal layer can be derived from the three-layer Fresnel equation at the condition for the minimum of the reflectance [Patskovsky et al 2003]:

$$
D_{o p t}=\frac{\lambda}{4 \pi} \frac{1}{\sqrt{\left|\varepsilon_{m}^{\prime}\right|} \cdot\left(1+\frac{\varepsilon_{d}}{2\left|\varepsilon_{m}^{\prime}\right|}\right)} \ln \left(\frac{4\left|\varepsilon_{m}^{\prime}\right| \sin (\varphi)}{\varepsilon^{\prime \prime}{ }_{m}}\right),
$$

where

$$
\tan \varphi / 2=\frac{\varepsilon_{p}}{\sqrt{\left|\varepsilon_{m}^{\prime}\right|\left(\varepsilon_{p}-\varepsilon_{d}\right)-\varepsilon_{p} \varepsilon_{d}}}
$$

These formulas will be used in Chapter 4 to find optimal metal's thickness for the proposed sensor.

\subsubsection{Excitation of SPR in an optical fiber}

Even though sensors currently based on the Kretschmann configuration can be relatively small, there has always been an attempt to realize SPR in optical fiber to produce a more compact sensor with remote sensing capabilities. Excitation of SPR in optical fibers is similar to the excitation of SPR in the prism configuration and it is based on the principle of Total Internal Reflection. Light propagating in the fiber core and cladding in the form of the modes experience TIR at the cladding-core and claddingexterior medium interfaces because of the difference between the refractive indices of the 
core, cladding materials and exterior medium. Different modes hit the cladding-core and cladding-exterior medium interfaces at different angles. Similarity between light propagating in the optical fiber via the total internal reflection and in prism configuration led to many successful attempts to realize SPR sensors in optical fibers.

Most of the realized fiber based SPR sensors work in the transmission regime where light from a broadband source is launched into one end of the fiber and is detected at another end (Figure 3.7). If plasmon resonance occurs and one of the modes couples to the SPW detected power at a wavelength of this mode, it will decrease resulting in a spike in the spectrum. To date, fiber SPR sensors work as wavelength encoded, but not as angle encoded which occurs in the prism configuration.

Fiber sensors can work in the reflection regime (fiber probe) as an alternative to the transmission regime. Generally, such a sensor consists of the fiber with a perfect cut and a layer of gold or another reflecting material at one end of the fiber which reflects light back towards the detector at the opposite end. Light propagating in the fiber is being altered by coupling to SPW if SPR occurs. The reflected light from the cut side is detected when traveling back. The design of the probes allows the sensor to be extremely compact, user-friendly and practical for in situ measurements in small spatial volumes. 


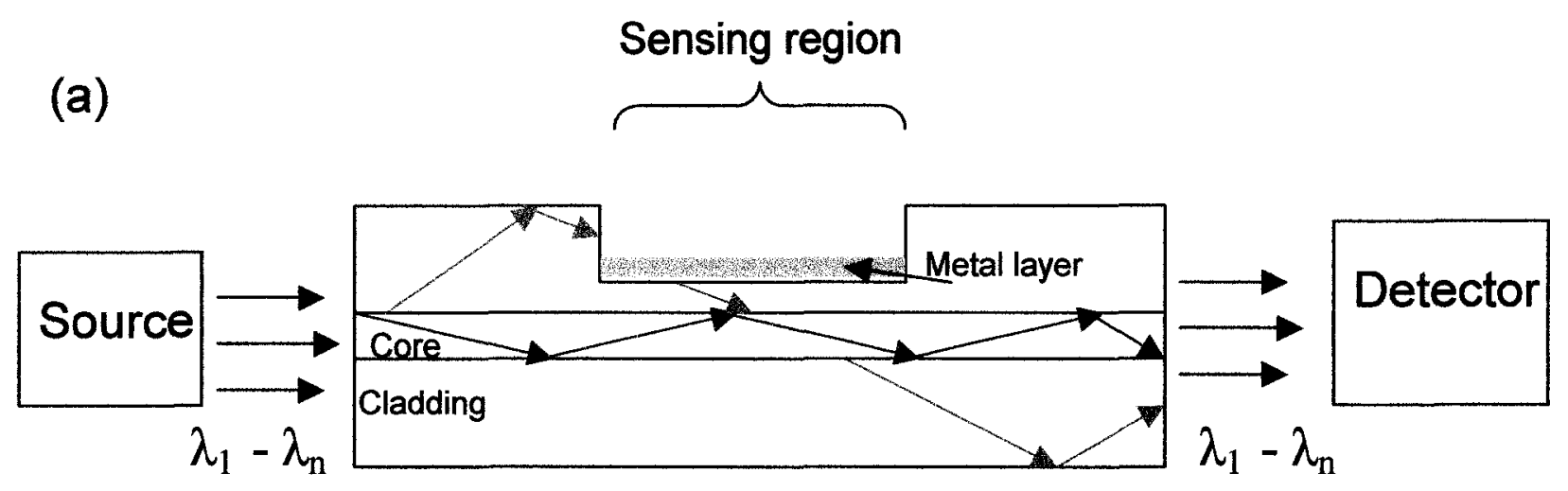

Sensing region

(b)

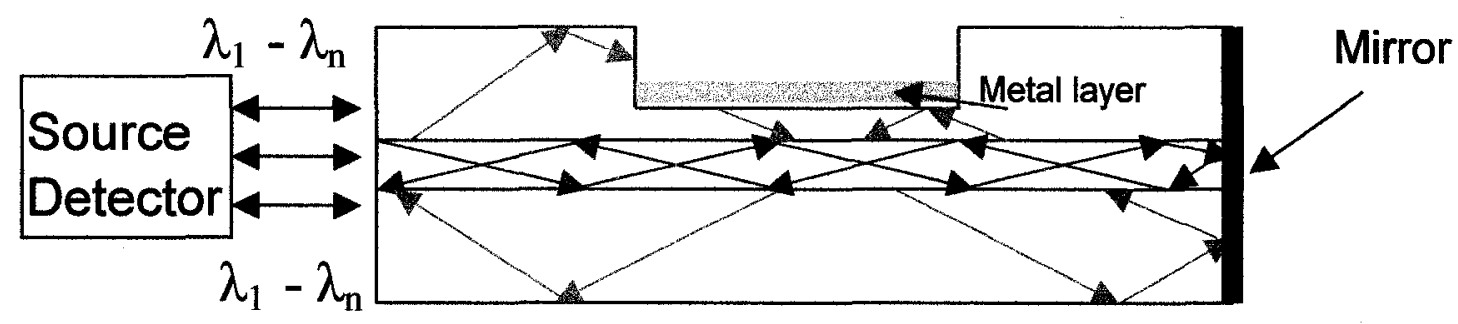

Figure 3.7. Diagram showing fiber SPR sensors working in the transmission regime (a) and reflection regime (b).

Even though the sensors described here work in different regimes, have different geometrical parameters and are suited for a variety of different applications, they can have similar sensing mechanisms. The SPR sensing mechanism is the most important element of the sensor design and defines such crucial sensing parameters as sensitivity, compactness, and robustness.

Unfortunately in standard telecommunication fiber (Corning SMF 28), the coupling mechanism corresponding to the prism configuration is not strong enough to excite a plasmon resonance. If the standard fiber is coated with gold or other SPR active metal and the matching condition is satisfied by the core mode, SPR will not be excited on the fiber surface because the strength of the resulting evanescent field from TIR at the core-cladding interface is too weak to cross the cladding and interact with the metal layer. Cladding modes can also be employed to excite SPR. However, a coupling mechanism 
must be provided to couple light from the core mode to the cladding modes. Consequently, there are no SPR sensors based on the standard single mode fiber without deformations and modifications. Existing approaches are based on the excitation of SPR by means of enhancing the evanescent field near the metal layer and can be divided in two groups.

In the first group of approaches, the fiber is deformed in such a way that the metal layer is located in proximity to the core and core-cladding interface. The evanescent field resulting from the core mode reflection at the core-cladding interface can excite SPR if it is strong enough to reach the metal layer. The metal layer can be located in proximity of the core-cladding interface by partially or completely removing the cladding. Typically, this can be achieved by creating a deep cut in the cladding [Jorgenson and Yee, 1993] (Figure 3.8) or by bending the fiber and polishing one of the fiber's sides [Homola, 1995; Tseng et al., 1997; Esteban et al., 2002; Lin et al., 2007] (Figure 3.9) otherwise by use of non-standard fiber such as D-type optical fiber [Gentleman et al., 2004]. Another accepted approach is to use a tapered fiber [Tubb et al., 1997; González-Cano et al., 2005] (Figure 3.10). In some approaches it has been suggested that the plain metal coating can be replaced by nanoparticle layers [Sharma and Gupta, 2005; Sharma and Gupta, 2006], which may improve the performance of SPR fiber sensors.

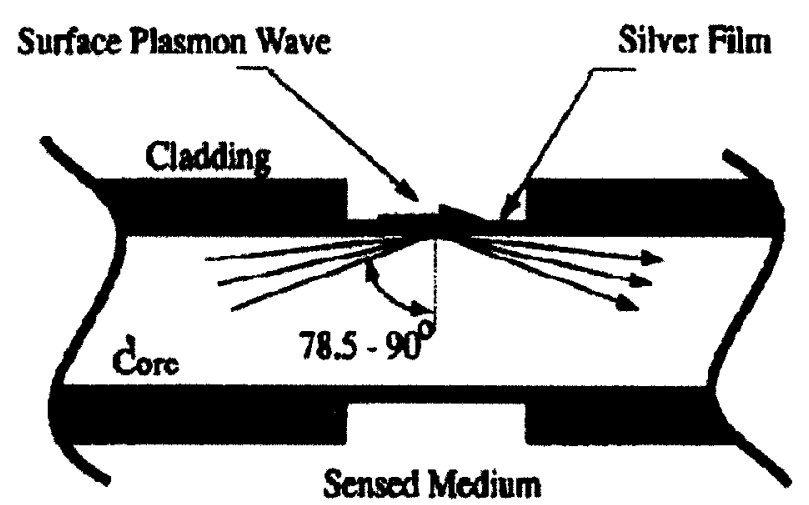

Figure 3.8 SPR sensor based on the fiber with deep cut in the cladding [Jorgenson and Yee, 1993]. 


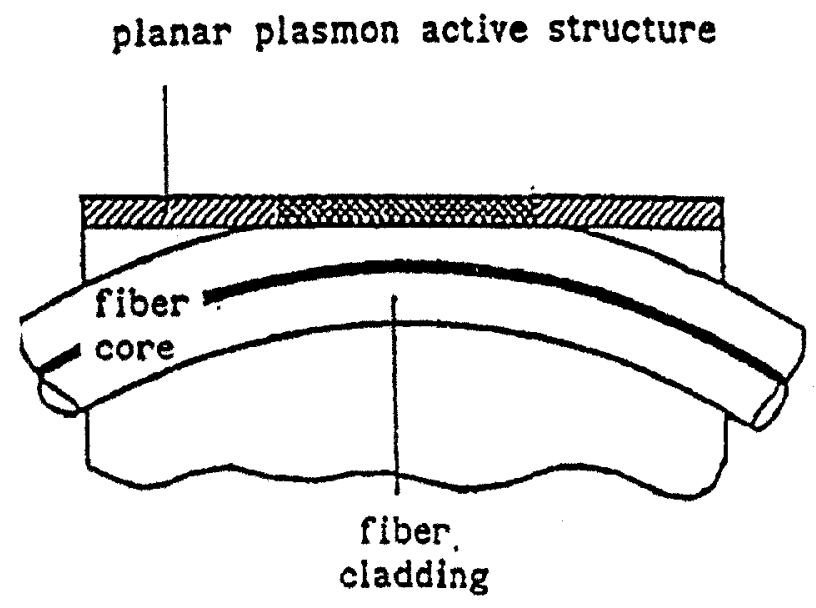

Figure 3.9. SPR sensor based on the bent and side-polished fiber [Homola, 1995].

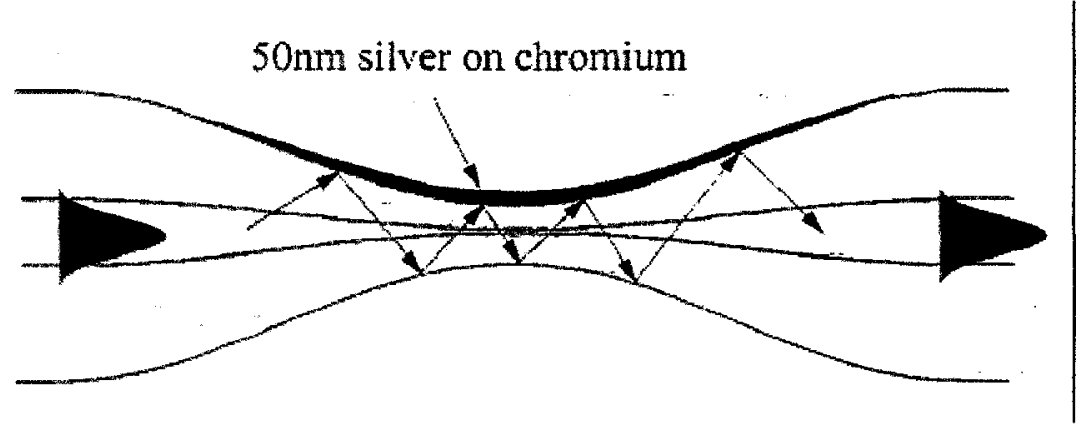

Figure 3.10. SPR sensor based on the tapered fiber [Tubb et al., 1997].

Primarily, the approaches mentioned here were proposed in the 90s. The minimal detectable index resolution for these types of SPR sensors is in the range of $10^{-4}$ to $10^{-6}$, which is close but does not exceed the resolution of prism-based SPR sensors. However, some of the researchers achieved extremely high resolution for fiber SPR sensors approximately $10^{-7}$ by precise control of the polarization of light and disturbances occurring during the experiment (which is far from the situation of real measurements making the experimental conditions artificial) [Slavik et al., 2007] and by precise control during metal deposition and the creation of the semi-cylindrical metal coatings which are able to support different hybrid plasmon waves [Monzon-Hernandez and Villatoro , 2006].

One of the disadvantages of the sensors mentioned here (which belong to the first group) is the fact that SPR is getting excited in both multi-mode and single-mode based fibers by un-controlled fields. As a result, experimental results show broad and ill- 
defined SPR resonances in the spectra that decrease the overall sensor performance. From another perspective, these sensors are based on modified, non-standard fibers that may result in a highly fragile sensor with possibly a higher cost compared to sensors made of unmodified standard fibers.

The second group of approaches is relatively new. Fiber gratings are being used to couple light to a specific mode that generates an evanescent field at the core-metal or cladding-metal interface strong enough to excite SPR. He et al, (2006) proposed a LPG for coupling of the core mode to the co-propagating cladding mode with a specific value of the wave vector such that it can excite a SPR (Figure 3.11). Nemova and Kashyap, (2006) proposed to use a FBG to couple light to a back propagating core mode in a hollow core fiber (Figure 3.12) and in a fiber with non-standard geometrical parameters (core diameter is $26 \mu \mathrm{m}$ and cladding diameter is $30 \mu \mathrm{m}$ ). A small cladding coupled with a big core radius allows strong field overlap between the reflected back core mode and the SPW.

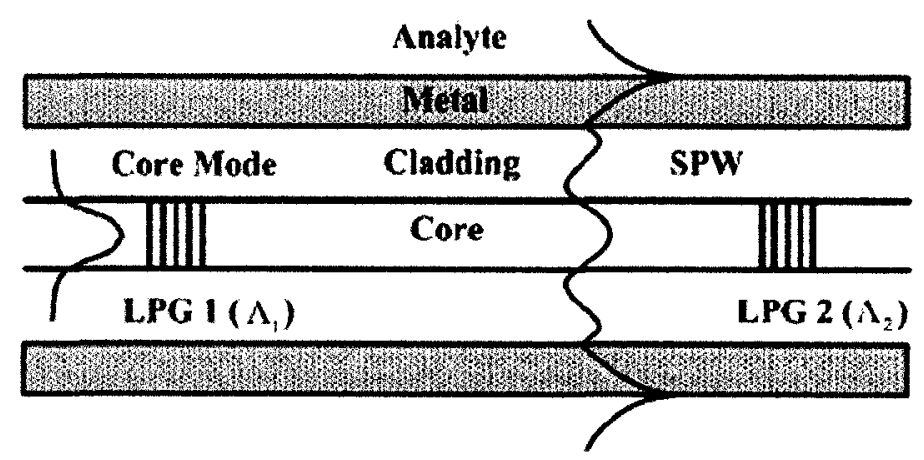

Figure 3.11 SPR sensor based on the fiber with LPG [He et al., 2006].

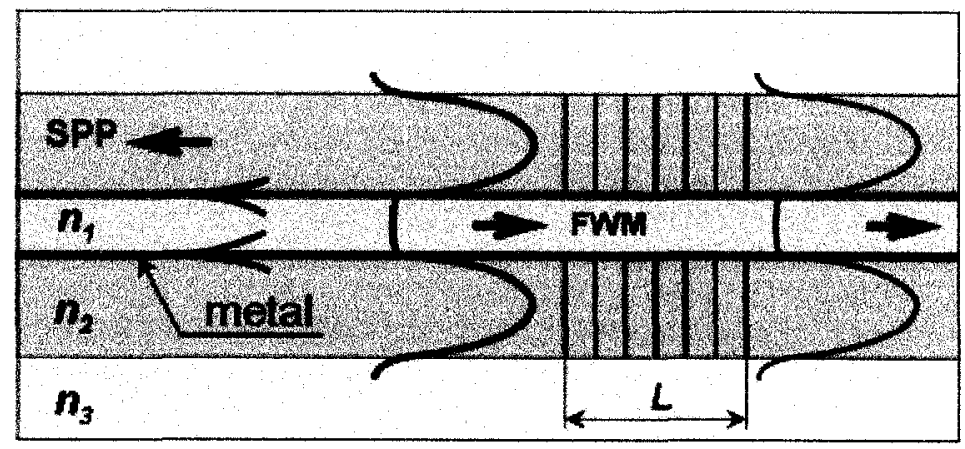

Figure 3.12. SPR sensor based on the hollow core fiber with FBG [Nemova and Kashyap, 2006]. 
Although these proposed approaches that employ LPGs and FBGs show a promising level of sensitivity (Nemova and Kashyap, (2006) predicted $300 \mathrm{pm}$ per $10^{-3}$ RIU), they have a couple of drawbacks. First, in the case of both LPG and FBG based sensors SPR excitement occurs because of coupling of only one certain mode to SPW that can be either a forward propagating cladding mode or backward propagating core mode. Use of only one mode limits the operating range of the sensor; however, this can be overcome by multiple writings of the gratings with different periods, making the sensor design more complicated. Second, the sensor's design with a FBG requires complicated fiber configurations. The length of FBGs in the proposed design by Nemova and Kashyap, (2006) is not very small and can vary from 2 to $10 \mathrm{~cm}$. Such parameters make the sensors design not as compact and simple as it could be implemented with standard optical fibers. In general, most of the proposed sensors based on fiber gratings were not tested experimentally and still remain as theoretical work.

Use of the tilted grating in the SPR sensor allows several (up to several hundred) cladding modes to be used for SPR excitement. This automatically solves such problems occurring with LPGs and FBGs based sensors that have limited operating range and bulk sensor geometry (at the time this research was conducted). Experimental work performed by Allsop et al, (2007) showed that a TFBG written in lapped fiber could achieve a high sensitivity ( $3365 \mathrm{~nm} /$ refractive index unit (r.i.u.)) and big operating range $\left(\Delta n_{d}=0.8\right.$ in aqueous mediums). Although this work is one of the most advanced in its field, it still possesses some disadvantages such as a wide SPR resonance $(>50 \mathrm{~nm})$ and the use of a modified highly fragile fiber.

Through this literature review, it can be concluded that many sensors, despite the high proposed sensitivity, have not yet been experimentally tested. Absence of the theoretical apparatus developed specifically for the circular geometry of the fiber supported SPR may result in a failure to control SPR and understand all the experimental results. Complications occurring during the manufacturing of complicated sensors may result in changes of the SPR matching condition and possibly a widening of SPR and as a consequence in a reduced sensitivity. The complicated sensor design may also limit the sensors to be widely recognized in the sensing field because of their fragility and complexity, which in turn raises manufacturing costs. The research conducted to date 
has shown that a lot has been accomplished in this field. However, a lot still can be done to develop new approaches and to improve the performance of the proposed sensors, in order to make them more robust and simplified. 


\section{Chapter 4. Proposed sensor design}

\section{Objectives:}

As described in Chapter 3 the current SPR sensors are based on complicated designs utilizing non-standard, modified fibers, however some of these sensors provide high sensitivities. The SPR fiber sensor approach described in detail in Chapter 4 utilizes the TFBG technology to provide a high sensitivity with no fiber deformations such as bends or cuts, resulting in a highly robust sensor. Section 4.1 introduces the proposed SPR sensor design and explains the principles of SPR excitation in optical fibers with TFBG. The theoretical background provided in Chapters 2 and 3 is used to find such proposed sensor's characteristics as sensitivity, operating range, depth of SPW penetration and optimal gold thickness. These calculations are presented in Section 4.2

\subsection{SPR fiber based sensor's principle}

The excitation of SPR in a single mode fiber with TFBG becomes possible through the coupling of light from the forward propagating core mode to the backpropagating core mode and a set of back-propagating cladding modes. Since the cladding modes are located in proximity to the cladding-metal interface, evanescent fields are generated through the TIR at the cladding-metal interface and are able to pass through the metal if it is thin enough and interact with the metal-exterior medium. If one of the back-propagating cladding modes has a wave vector that matches the wave vector of SPW that could exist at the outer metal surface, then the SPR is being excited (Figure 4.1). 


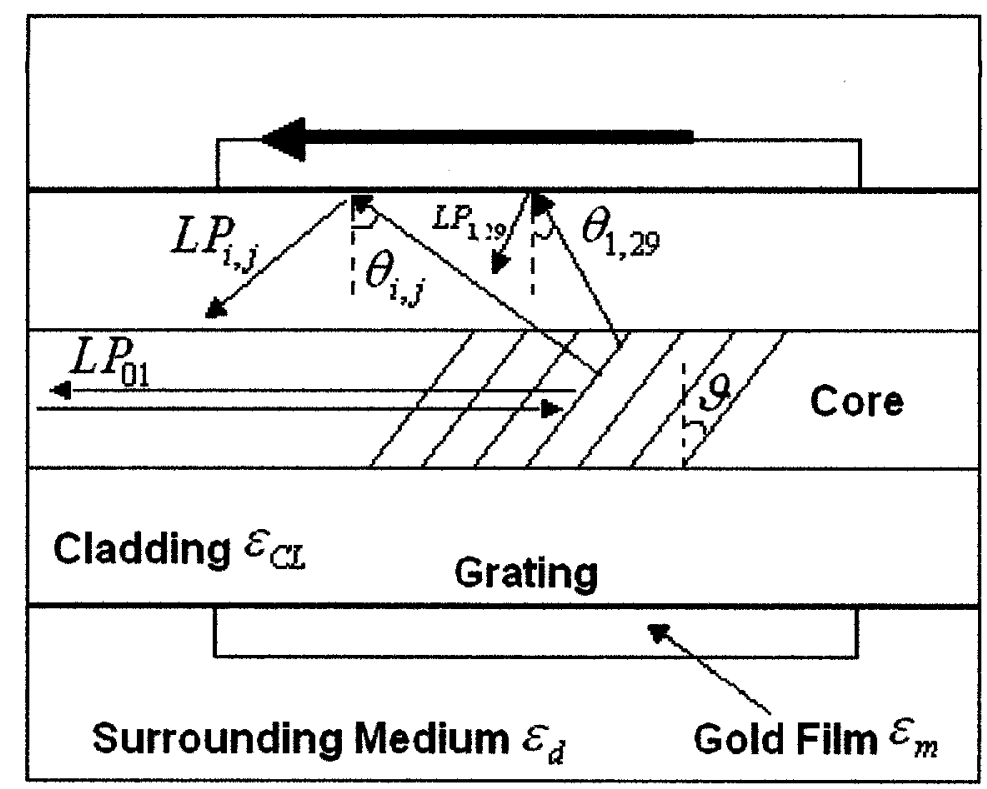

Figure 4.1. Schematic diagram of the proposed SPR sensor to show the principle of light coupling through TFBG into cladding modes and SPWs, consecutively.

Given that the theory that describes the excitation of SPWs in fibers with a circular geometry has not yet been completely developed, this work employs a theoretical approach developed for the prism configuration with a planar geometry described in Chapter 3. It is possible using this approach to predict SPR, however it is still not completely understood how accurate and how the SPR supported by the circular geometry will differ from the SPR supported by the planar geometry. These precautions also relate to the determination of the optimal metal thickness described in Chapter 3.

There are two main formulas to this approach. The first is the matching condition for the TFBG using equation 2.19 from Chapter 2 and the second is the matching condition for the SPR (Equation 3.37) from Chapter 3. They are used to find the sensor characteristics for SPR excitation as follows:

$$
\begin{aligned}
& n^{\text {core }}{ }_{\text {eff }}^{i}+n^{\text {clad }}{ }_{\text {eff }}^{i}=\frac{\lambda^{i}}{\Lambda /(\cos \vartheta)}, \text { and } \\
& \sqrt{\varepsilon_{p}} \sin (\theta)=\operatorname{Re}\left\{\sqrt{\frac{\varepsilon_{m} \varepsilon_{d}}{\varepsilon_{m}+\varepsilon_{d}}}\right\},
\end{aligned}
$$


where $\vartheta$ is the tilt angle of the grating and $\theta$ is the angle which is formed by the cladding mode reflecting at the cladding-metal interface (Figure 4.1).

The connecting element between these two equations is:

$\sqrt{\varepsilon_{p}}=n^{\text {clad }}\left(\lambda^{i}\right)$

Therefore by substitution of equation 4.3 and 4.1 into 4.2 it is possible to find the refractive index of the sensed medium $n_{d}$, which can support SPW on the surface of the gold-coated fiber:

$n_{d} \sqrt{\varepsilon_{p} \varepsilon_{m} \sin \left(a \sin \left(\frac{\frac{\lambda^{i}}{\Lambda / \cos \vartheta}-n_{e f f}^{c o r e}}{n^{c l a d}\left(\lambda_{i}\right)}\right)\right)}$,

Note that all refractive indices and permittivities must be evaluated at the wavelength of the resonance $\lambda_{p}$.

\subsection{Calculation of the SPR parameters and excitation conditions}

\subsubsection{Refractive index of sensed medium $n_{d}$ and operating range $\Delta n_{d}$}

Substituting the parameters of specific fiber and grating into equation 4.4 makes it possible to determine if both the SPR can exist on the surface of the metal coated fiber and the range $n_{d}$ supported by the SPR $\Delta n_{d}$.

For the experiments presented, a grating with a tilt angle of $4^{\circ}$ was written in a standard telecom single mode fiber (CORNING SMF 28), which was later coated with gold. This fiber has a core diameter of $8.2 \mu \mathrm{m}$, a cladding diameter of $125 \mu \mathrm{m}$ [Corning, 2002], and the refractive indices of the core and cladding are 1.4508 and 1.4440 respectively at a wavelength of $1550 \mathrm{~nm}$ (for other wavelengths these values follow the Sellmeir's equation for silica [Malitson, 1965]). The metal's dielectric function was derived based on the data provided by Johnson and Christy, (1972). The effective indices 
of the core and cladding modes for specific wavelengths were calculated with the aid of the OptiGrating software (Version 4.2.1. (BZ3736)).

The period of the grating used in the experiments was found with help of equation 2.20 from Chapter 2:

$$
n_{\text {eff }}{ }^{q}+n_{\text {eff }}{ }^{m}=\frac{\lambda^{m}}{\Lambda /(\cos \vartheta)},
$$

For this calculation the Bragg wavelength $\lambda^{B}$ was determined from the grating spectrum measured after the grating fabrication was complete (Figure 4.2). According to this calculation the Bragg resonance would occur at a wavelength of $\lambda^{B}=1549.9 \mathrm{~nm}$ in the grating with a $4^{\circ}$ tilt angle if only the period of the grating would be $\Lambda=0.53419 \mu \mathrm{m}$.

When taking into account the obtained characteristics of the fiber and grating while at the same time assuming that SPR can only be excited by cladding modes in the wavelength range of $1520.947 \mathrm{~nm}$ to $1546.61 \mathrm{~nm}$ (almost all cladding modes appear in this range in Figure 4.2 except for the 'ghost' mode at $1548 \mathrm{~nm}$ which is guided very near the core). It was found from equation 4.4 that SPRs can be excited on the outer surface of the metal coating if the refractive index of the sensed medium $n_{d}$ lies within the range $\Delta n_{d}=1.3967$ to 1.4442 . 


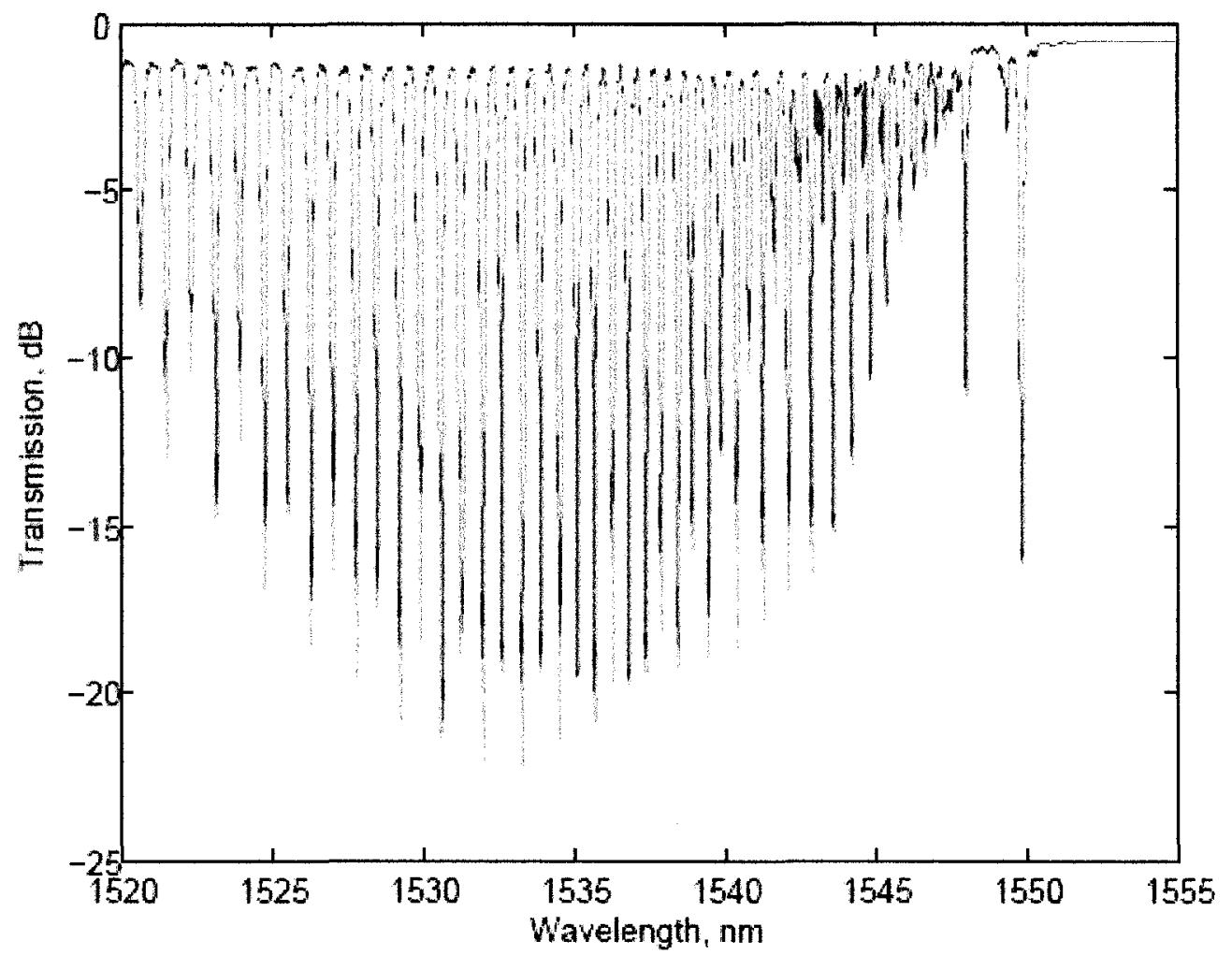

Figure 4.2. Transmission spectrum of the grating that was used in experiments (tilt angle is $4^{\circ}$ ).

\subsubsection{Proposed sensitivity}

Since the sensor is "read" by a wavelength interrogation system its sensitivity can bedetermined as change in the SPW wavelength $\lambda_{p}$ position relative to the change in refractive index of the sensed medium $n_{d}$ :

$$
S=\frac{\Delta \lambda_{p}}{\Delta n_{d}},
$$

When considering the wavelength range of the cladding modes that are suppose to support SPR (1520.947 $\mathrm{nm}$ to $1546.61 \mathrm{~nm}$ ) and operating range $\Delta n_{d}$ obtained in the previous paragraph, sensitivity $\mathrm{S}$ can be estimated as (linear approximation):

$$
S=\frac{(1546.61-1520.947) n m}{1.442-1.3967 r \text { i.u. }}=543.5 \mathrm{~nm} / \text { r.i.u }
$$


This value is similar to that calculated by Nemova and Kashyap, (2006) for SPR sensors based on hollow core fibers with FBG as the coupling element.

\subsubsection{SPW penetration depth into sensed medium $L_{p d}$, metal $L_{p m}$ and length of propagation $L$}

The penetration depth of the SPW in the both sensed medium $L_{p d}$ and gold $L_{p m}$ can be found using equations 3.31 to 3.34 of Chapter 2 :

$$
\begin{aligned}
& \gamma_{m}=i \frac{\omega}{c} \frac{\varepsilon_{m}}{\sqrt{\varepsilon_{m}+\varepsilon_{d}}}, \\
& \gamma_{d}=i \frac{\omega}{c} \frac{\varepsilon_{d}}{\sqrt{\varepsilon_{m}+\varepsilon_{d}}}, \\
& L_{p m}=1 / \operatorname{Re}\left\{\gamma_{m}\right\}, \\
& L_{p d}=1 / \operatorname{Re}\left\{\gamma_{d}\right\} .
\end{aligned}
$$

By substituting the range $\Delta n_{d}$ into equations 3.31 to 3.34 , calculations indicate that the depth of penetration of the plasmon field in the dielectric varies from $27.36 \mu \mathrm{m}$ to $13.12 \mu \mathrm{m}$ at wavelengths in the range $1520.947 \mathrm{~nm}$ to $1546.61 \mathrm{~nm}$. The penetration depth in the gold varies from $0.4838 \mu \mathrm{m}$ to $0.2388 \mu \mathrm{m}$ for the same wavelength range. This indicates that plasmons can't be excited on the outer side of the gold coating if the gold thickness exceeds a few hundred nanometres.

By substitution of the same wavelength and refractive index ranges in equation 3.24 it is possible to find the propagation length of the SPW $L$ :

$L=\frac{1}{2 \beta^{\prime \prime}}$, where $\beta^{\prime \prime}$ can be found from (3.22).

The length $L$ varies from $49.25 \mu \mathrm{m}$ to $46.48 \mu \mathrm{m}$. The grating is much longer $\left(10^{-2}\right.$ m) than the SPW propagation length that ensures excitation of SPW at several locations at the fiber's surface.

\subsubsection{Optimal metal thickness calculations}

The optimal metal layer thickness for the given metal and dielectric's parameters can be found using Equations 3.38 and 3.39: 


$$
D_{o p t}=\frac{\lambda}{4 \pi} \frac{1}{\sqrt{\left|\varepsilon_{m}^{\prime}\right|} \cdot\left(1+\frac{\varepsilon_{d}}{2\left|\varepsilon_{m}^{\prime}\right|}\right)} \ln \left(\frac{4\left|\varepsilon_{m}^{\prime}\right| \sin (\varphi)}{\varepsilon^{\prime \prime}{ }_{m}}\right),
$$

where

$$
\tan \varphi / 2=\frac{\varepsilon_{p}}{\sqrt{\left|\varepsilon_{m}^{\prime}\right|\left(\varepsilon_{p}-\varepsilon_{d}\right)-\varepsilon_{p} \varepsilon_{d}}} .
$$

Figure 4.3 displays plot of optimal gold thickness as a function of refractive index of the sensed medium $n_{d}(1.35$ to 1.5$)$ for two wavelengths $1524.5 \mathrm{~nm}$ and $1550 \mathrm{~nm}$.

This calculation was performed for SPR occurring where the gold layer is sandwiched between two dielectrics: silica dioxide $\left(\varepsilon_{p}\right)$ and an arbitrary sensed medium $\left(\varepsilon_{d}\right)$ (Lorentz model of sensed medium was omitted for the simplicity of calculations).

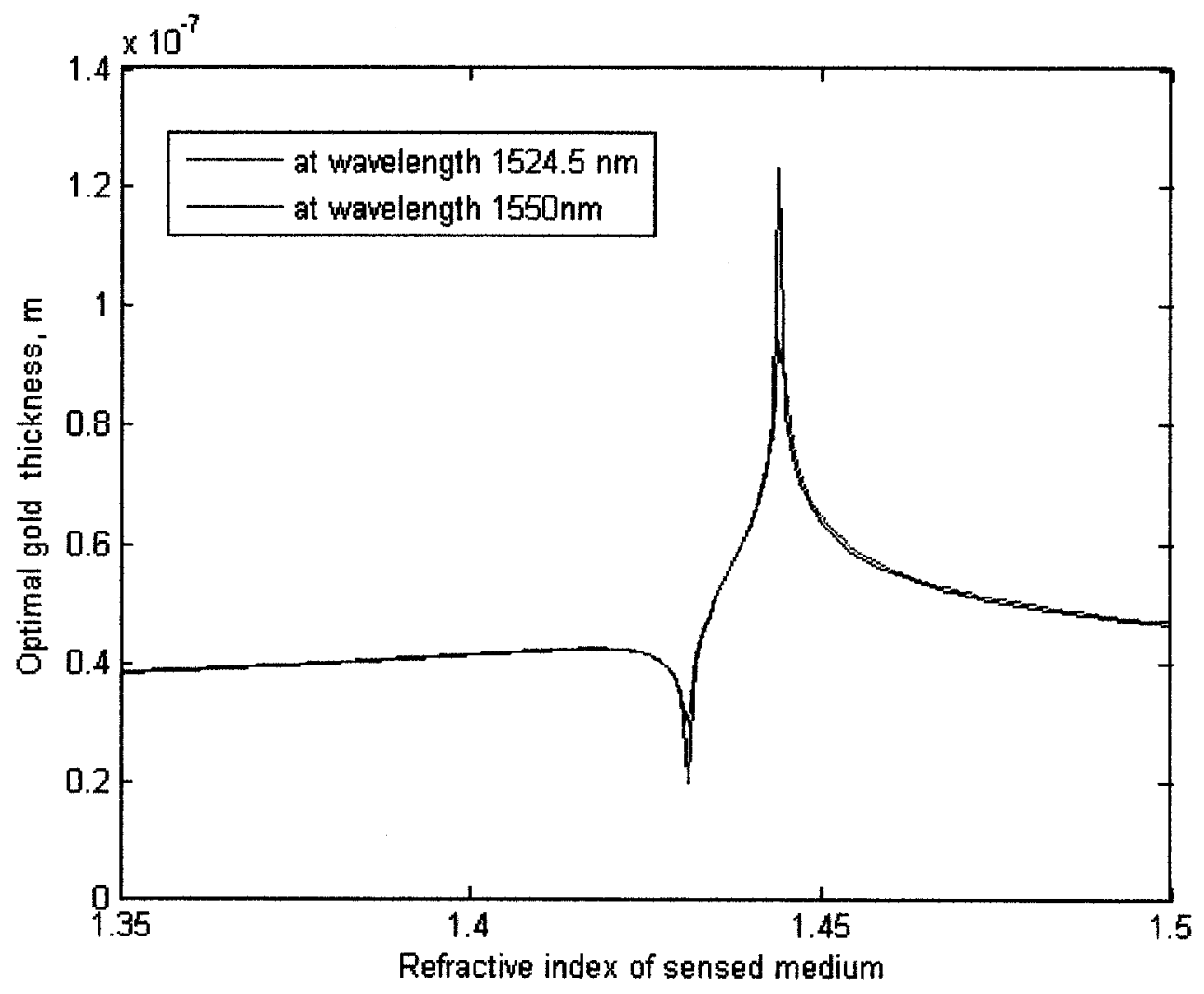

Figure 4.3. Optimal gold layer thickness. 
It is apparent from Figure 4.3 that the optimal thickness varies from $20 \mathrm{~nm}$ to 120 $\mathrm{nm}$ and is most sensitive to the refractive index of the sensed medium $n_{d}$. The significant changes of optimal thickness, especially at values approaching refractive index of silicon dioxide, could be a possible subject for further research. 


\section{Chapter 5. Manufacturing of the SPR sensor}

\section{Objectives:}

Even though the sensor has a simple design and requires just a piece of fiber with a TFBG written in its core, plus a metal coating, the manufacturing process requires time and effort (especially for TFBG fabrication). The fabrication process can be divided into several steps: hydrogen loading, writing of the grating, annealing and gold deposition. Each of these steps is discussed in subsequent sections of this chapter.

\subsection{Fiber and Grating}

In this work a standard single mode fiber (CORNING SMF28) was used as a base for the SPR sensor. The TFBG as a key sensor element was written in a piece of fiber and was treated subsequently as an independent sensor. The fabrication of TFBGs was performed by Albane Laronche in July 2006 in the Laboratory for Laser Induced Photonics Structures.

Hydrogen loading: Prior to writing the TFBG, the fiber was left in hydrogen to soak and absorb gas in order to increase its photosensitivity. The fiber was left for 14 days in hydrogen until the process of the gas absorption was saturated. The gas absorption was facilitated by a high atmospheric pressure (2500 psi).

Tilted grating: Before gratings were written in the fiber, the coating (also known as overlay) was removed and the cladding was cleaned with methanol. The fiber gratings were fabricated by using a side-writing technique where only one laser beam was used and an interferometric pattern was formed by illuminating the fiber through a phase mask (Figure 5.1). The piece of fiber should be positioned very close to the phase mask, within $100 \mu \mathrm{m}$. If the fiber together with the mask is positioned at $90^{\circ}$ relative to the axis of light propagation, the grating is not going to have any tilt. Any small rotation of fiber around this axis results in the appearance of a tilt angle. In these experiments only gratings with small tilt angles of $4^{\circ}$ and $6^{\circ}$ were tested. A $\mathrm{KrF}$ excimer laser with operating wavelength $248 \mathrm{~nm}$ was used to write the gratings. 


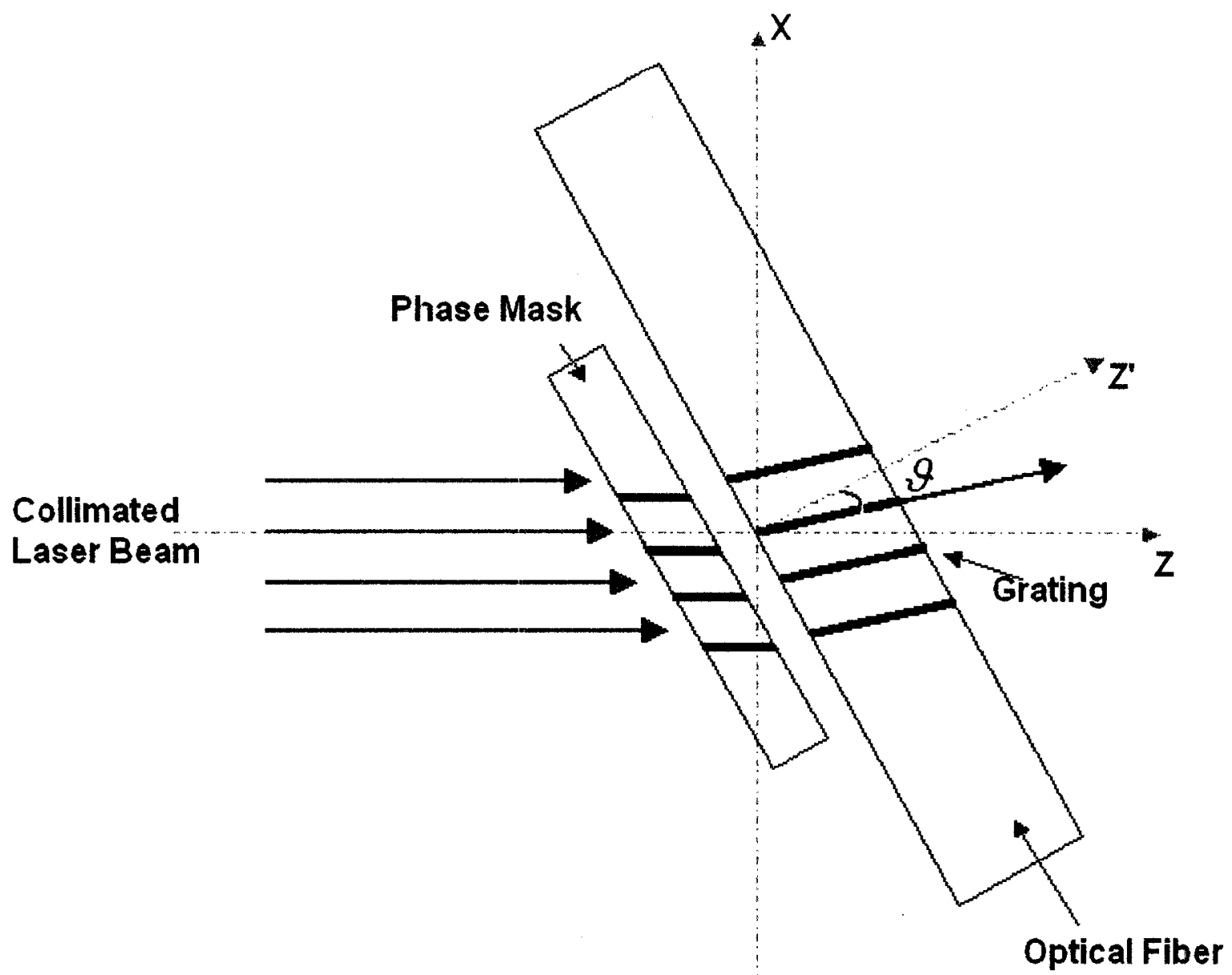

Figure 5.1. The principle of the side-writing technique.

Annealing: Annealing is a process aimed to stabilize the gratings by removing the most thermally unstable part of the UV-induced refractive index modulation. The first part of annealing is done with a heat gun at temperatures approaching $400^{\circ} \mathrm{C}$ for less than one minute. The second part requires heating of the fiber in an oven for 12 hours at a temperature of $120^{\circ} \mathrm{C}$ in order to remove the remaining hydrogen.

\subsection{Gold coating}

Gold in comparison to other SPR active metals is more chemically stable, provides the possibility of binding various films on its surface and possesses a good biocompatibility [Echegoin et al., 2006]. This makes it very attractive for future research involving biosensors where different molecules should be immobilized on the metal 
surface. Given these attractive characteristics it was decided that gold would be used for these experiments.

Since proposed sensor was planned to be tested over the operating range $\Delta n_{d}=1.3967$ to 1.4442 and calculations revealed that optimal gold thickness for this operating range varies from $20 \mathrm{~nm}$ to $65 \mathrm{~nm}$, sensors were fabricated with 20,30 and $50 \mathrm{~nm}$ of metal layer thickness.

For these preliminary experiments, the gold coating was deposited in a small-scale sputtering chamber (Polaron Instruments model E5100). According to specifications, a gold layer of $20 \mathrm{~nm}$ in thickness on a flat sample requires 1 min of deposition at a pressure of 0.1 Torr, a potential difference of $2.5 \mathrm{kV}$, and $18-20 \mathrm{~mA}$ of sputter current.

Typically during a single deposition, a bare piece of the fiber is positioned parallel to the sputtering target, hence only one side of the fiber is being coated. Given that the fiber has a round geometry it was very desirable to have a uniform coating over the entire surface. To achieve this, two depositions were consecutively done on opposite sides of the fiber. In such conditions, the gold thickness around the fiber is unlikely to be uniform and to correspond to the value obtained on a flat substrate (Figure 5.2).

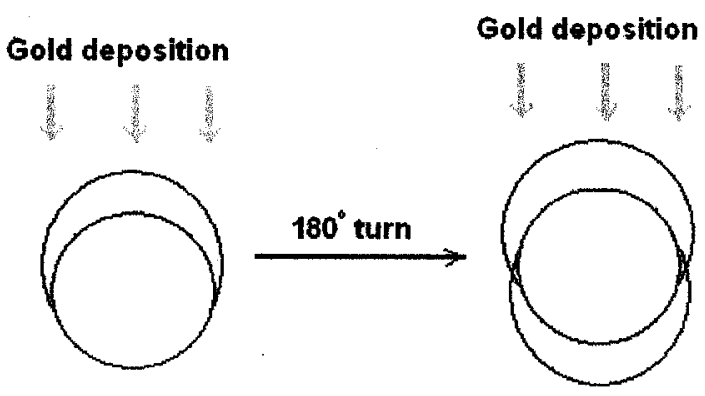

Figure 5.2. Principle of gold deposition in the chamber.

\subsection{Sugar solutions}

Each experiment was performed in air, water and sugar solutions. It was found that the sugar solutions would work better than a set of calibrated refractive index liquids available in the laboratory because it is possible to create solutions with very small 
changes in refractive index. To monitor the refractive index of the sugar solutions during the experiments, an Abbe refractometer was used that operated at $589.3 \mathrm{~nm}$ wavelength. 


\section{Chapter 6. Experimental results}

\section{Objectives}

This chapter presents the experimental results obtained with TFBG-SPR sensor. Section 6.1 introduces the experimental setup and conditions in which experiments were conducted.

The whole experimental work can be roughly divided into two parts:

1) testing of the sensor with a thin metal coating $(20 \mathrm{~nm})$; and

2) testing of the sensor with a thicker metal coatings (30 and $50 \mathrm{~nm}$ ).

Section 6.2 presents results related to the testing of the sensor with a thin metal coating. This sensor resulted in a strong SPR effect, hence its performance was studied in detail. Subsections of Section 6.2 include information about the sensor sensitivity, the SPR resonance width along with the grating's transmission spectra in different solutions. An investigation of the gold film quality and uniformity with help of AFM and SEM is also reported.

Section 6.3 describes the testing of the sensors with a thick metal coating. The Chapter concludes with a detailed discussion of the obtained results.

\subsection{Experimental setup}

The experiments involved connecting the fiber sample to a tunable laser source $(1520 \mathrm{~nm}$ to $1570 \mathrm{~nm})$ and to the detector (JDS Uniphase SWS-OMNI) at the opposite end (Figure 6.1). The shown experimental results are obtained from the average of four measurements in the orthogonal polarization states. The grating (sensing region) was located in the middle of the fiber sample and did not exceed a length of $0.5 \mathrm{~cm}$. Two glass slides were used to enclose the fiber (grating) within the sugar solution. One slide was fixed on the table with help of tape and several drops of sugar solution were applied to the surface of the slide immersing the fiber in the solution. The second slide was used to stabilize the position of the fiber ensuring it was immersed and there was no movement during the measurement of the transmission spectrum. After each of the experiments the fiber and glass slides were thoroughly cleaned with the help of water. The experiments were performed on a table that was not protected against vibration. 


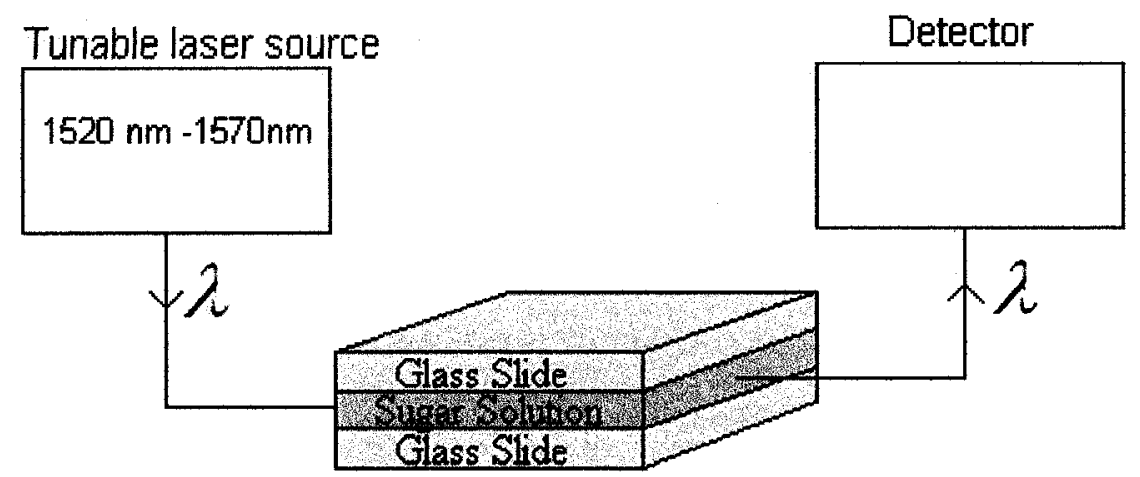

Figure 6.1. Experimental setup.

\subsection{Experimental results for grating with a $20 \mathrm{~nm}$ thick gold coating}

\subsubsection{Surface Plasmon Resonance (SPR)}

Figure 4.2 presented in Chapter 4 shows a transmission spectrum for the uncoated grating measured in air. The transmission spectrum has a Bragg resonance at $1549.9 \mathrm{~nm}$, next to it is a 'ghost' resonance and there are approximately 50 deep cladding mode resonances that appear between $1520 \mathrm{~nm}$ and $1546 \mathrm{~nm}$. Figure 6.2 shows the transmission spectrum measured in the air for the same grating, but with a gold coating (gold layer thickness is $20 \mathrm{~nm}$ ). It can be seen that by adding the metal coating, small changes occur to the spectrum and resonances becomes not as deep.

The gold-coated grating was further immersed in the sugar solutions with refractive indices within the range $\Delta n_{d}=1.3967$ to 1.4442 . This resulted in a change in the transmission spectrum with the appearance of strong resonances at different wavelengths. The spectrum displayed in Figure 6.3 shows the results for the gold-coated grating immersed in a sugar solution with $n_{d}=1.4378$. A deep resonance appeares in the middle of the spectrum and consists of several cladding mode resonances with increased amplitude that are not characteristic for the spectrum of the uncoated fiber immersed in the same sugar solution (Figure 6.4).

The experiment was then repeated with gradual changes in the concentration of sugar. It was found that a deep resonance 'moves' across the spectrum depending on the sugar concentration. Because of the absence of this resonance in the spectrum of the 
uncoated fiber immersed in the same sugar solutions and the possibility to predict it with SPR theory, this anomalous resonance was identified to originate from a SPR.

According to calculations in Section 4.2.1 the operating range for proposed sensor should be $\Delta n_{d}=1.3967$ to 1.4442 . During the experiments the grating was tested in a smaller range of the refractive index due to complications that likely occurred during the experiments. It was established that for certain values of $n_{d}$ of the sugar solution the SPR was always excited at wavelength shorter than it was predicted. Overall the experimentally obtained results showed that the operating range for this grating was $\Delta n_{d}=1.4211$ to 1.4429 with a shift towards a higher refracted index by 0.026 r.i.u compared to the predicted value (this issue will be discussed in Section 6.6.2).

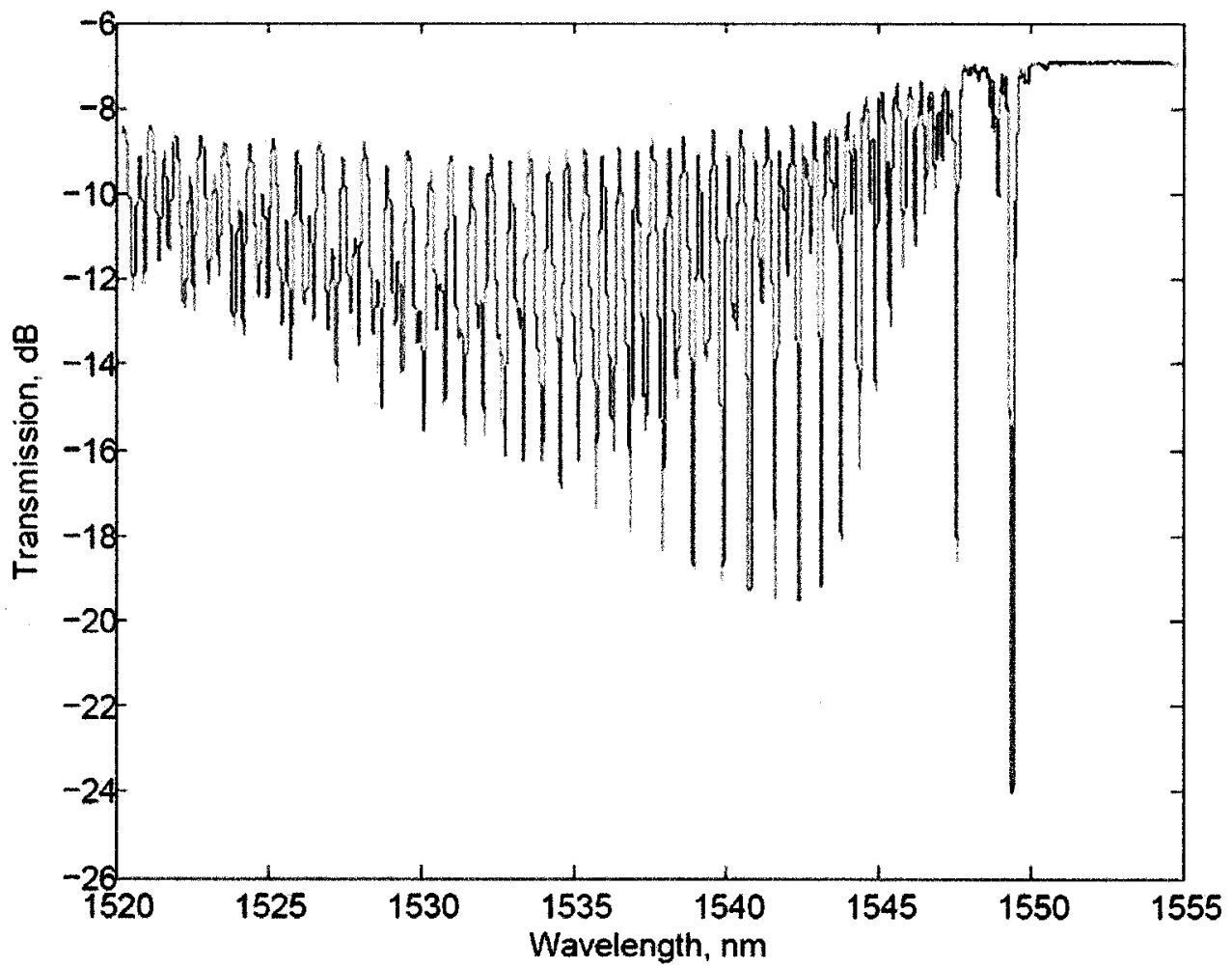

Figure 6.2. Transmission spectrum of the gold coated grating with a $4^{\circ}$ tilt measured in the air. 


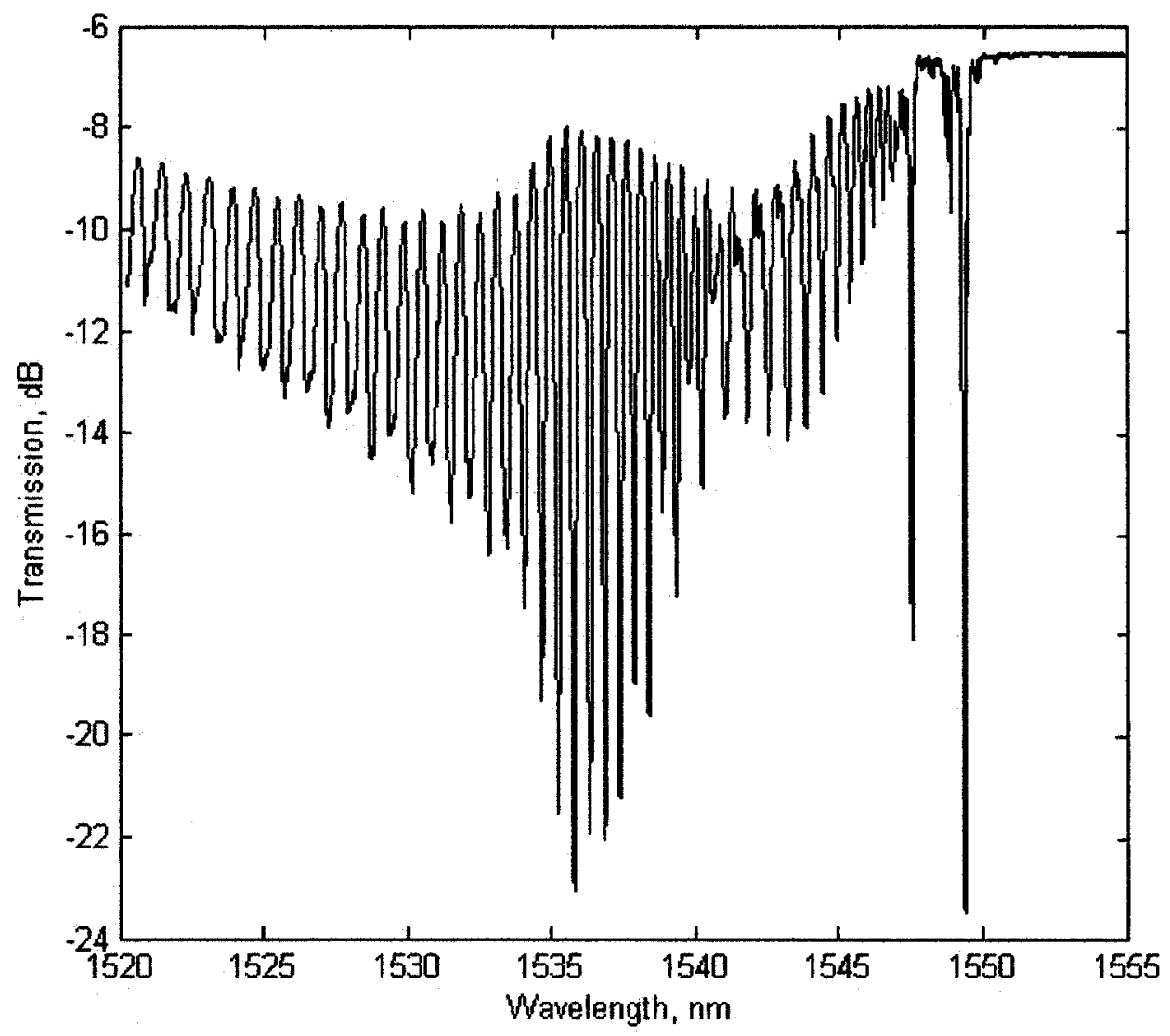

Figure 6.3. Transmission spectrum for the gold coated fiber immersed in the sugar solution with a refractive index of $\mathbf{n}_{\mathrm{d}}=1.4378$. 


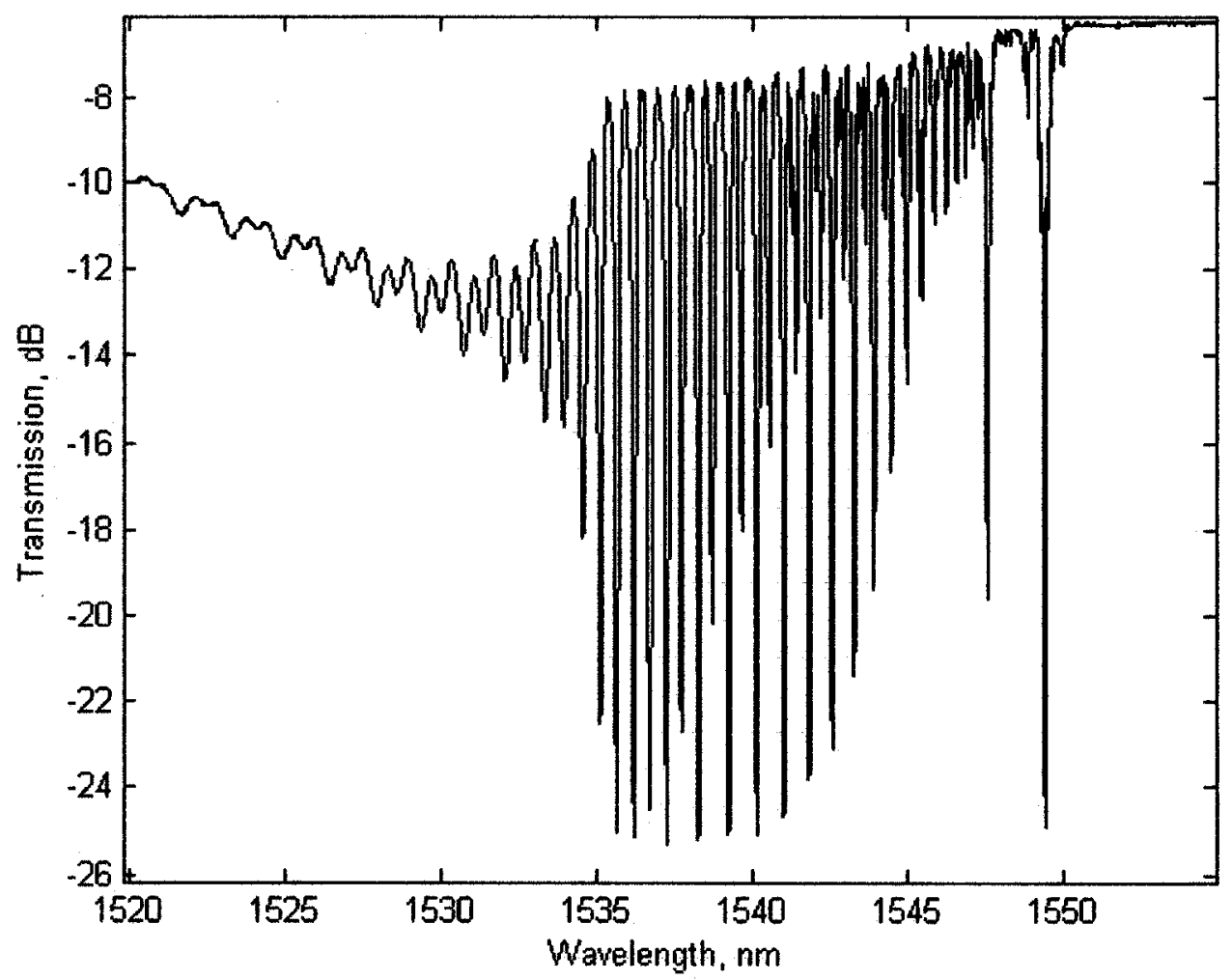

Figure 6.4. Transmission spectrum for an uncoated fiber immersed in the sugar solution with a refractive index of $n_{d}=1.4378$.

Figure 6.5 shows several transmission spectra for the same grating immersed in different sugar solutions. The figure indicates that a decrease in sugar concentration resulted in a blue shift in the SPR wavelength position. The SPR resonance changes not only the wavelength, but also the amplitude of the resonance. For example, the spectrum shown with a dark blue color (sugar solution immersion of $\mathrm{n}_{\mathrm{d}}=1.4211$ ) has SPR amplitude, which is not as large as the amplitude of resonance shown in the light blue color (sugar solution immersion of $n_{d}=1.4499$ ).

Furthermore it was found that the spectrum of the grating immersed in a relatively low sugar concentration has two SPR resonances (Figure 6.6) instead of one. The current model based on a planar geometry fails to explain this second resonance. 

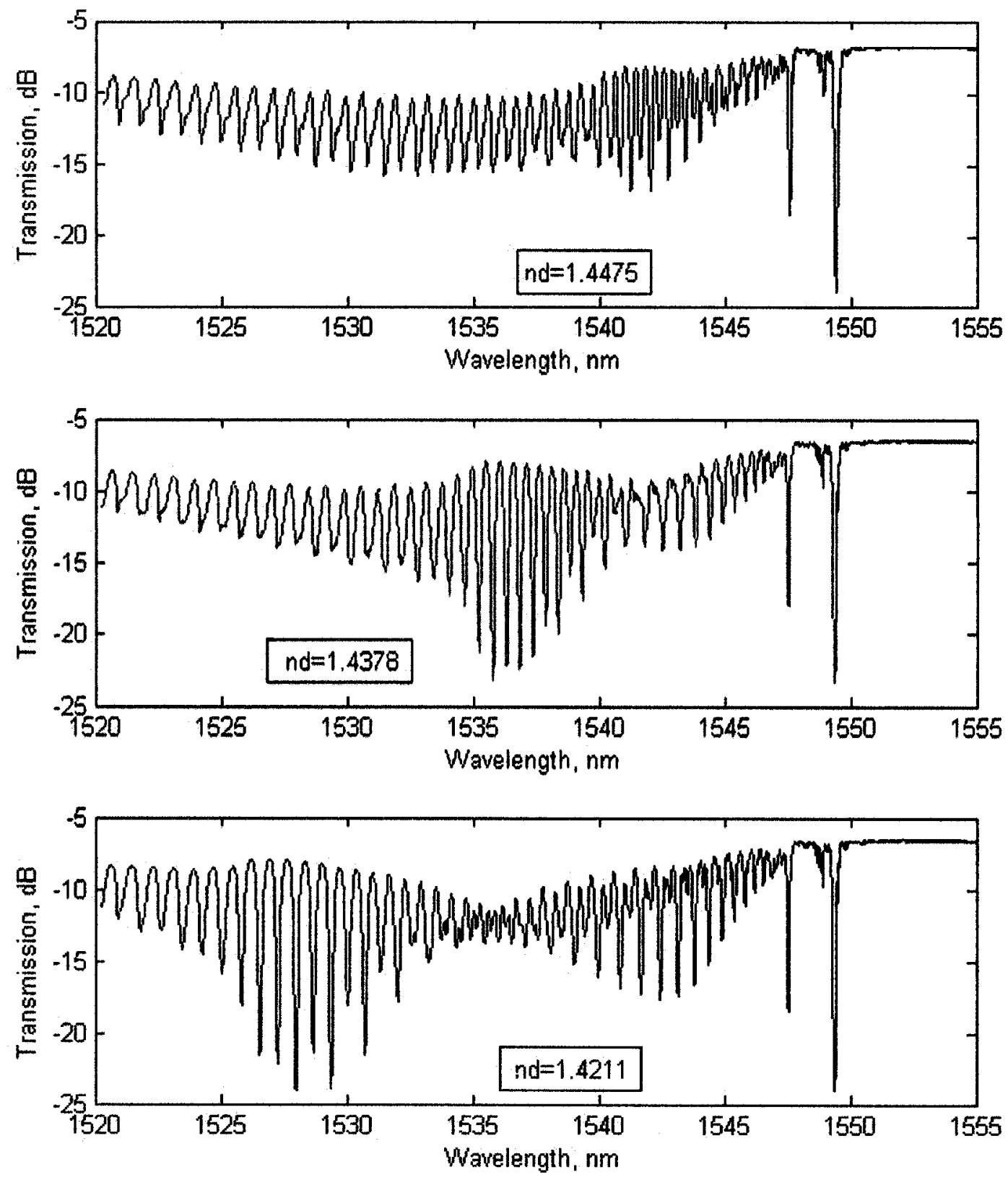

Figure 6.5. Transmission spectrum of the same grating as in Figure 6.3 immersed in sugar solutions with different $\mathrm{n}_{\mathbf{d}}$. 


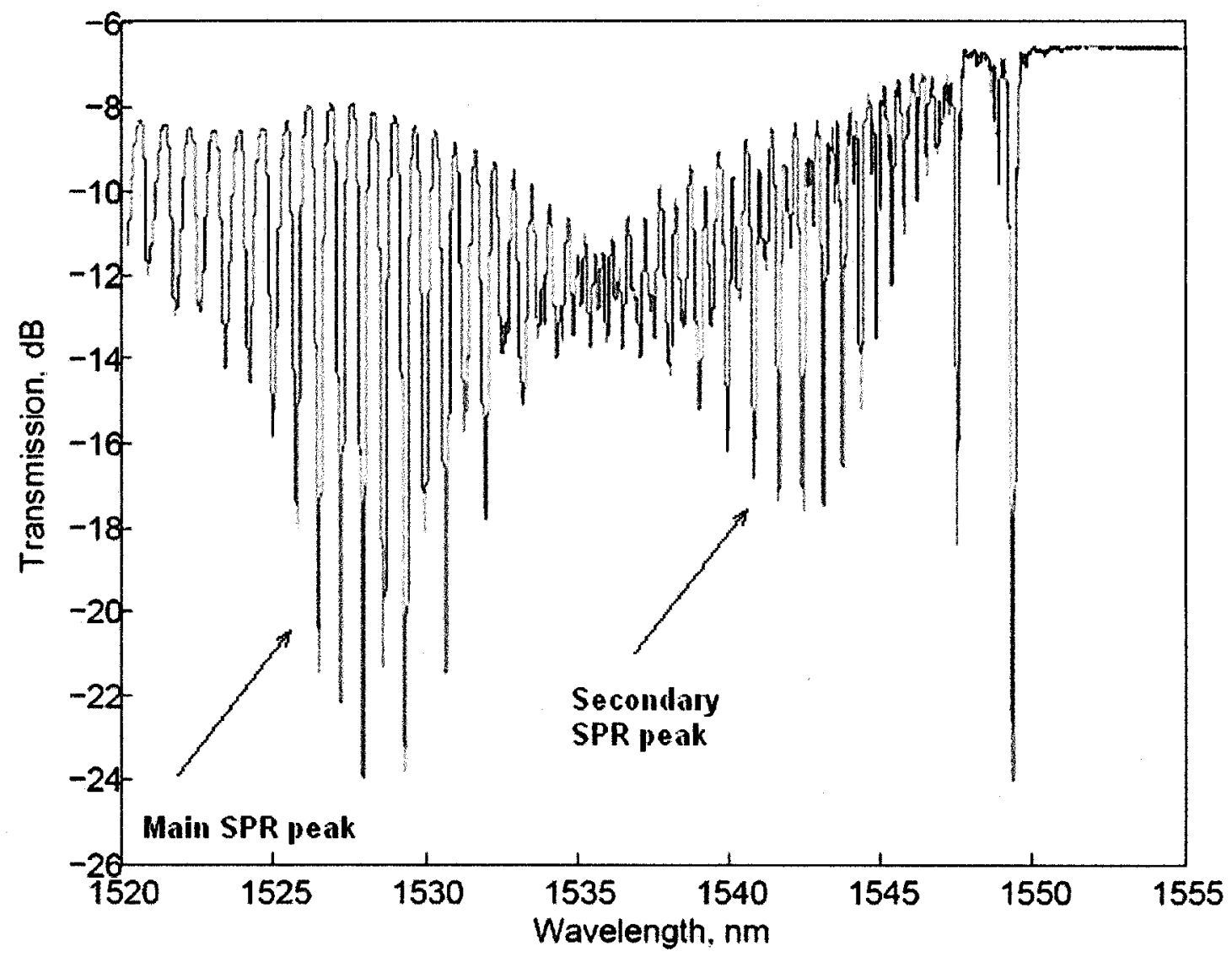

Figure 6.6. Transmission spectrum for grating immersed in the sugar solution with refractive index $\mathrm{n}_{\mathrm{d}}=1.4211$.

\subsubsection{Sensitivity}

In order to evaluate the sensor's performance, the SPR wavelength $\lambda_{p}$ was plotted as a function of the refractive index of sensed medium $n_{d}$. Sensitivity, determined as the change of the plasmon wavelength $\lambda_{p}$ relatively to the change of the refractive index of the sensed medium $n_{d}$, can be found as a slope of the fitting curve of the data in Figure 6.7. Data was approximated by a straight line with a slope of $454 \mathrm{~nm} / \mathrm{r}$.i.u. (norm of residuals was $5.284 \mathrm{~nm}$ ). This sensitivity $(454 \mathrm{~nm} /$ r.i.u.) is close to the predicted value of $543.5 \mathrm{~nm} /$ r.i.u (Section 4.2.2).

Detection accuracy $a$ determines how accurately and precisely the instrument can measure the signal. Detection accuracy and sensitivity can be used to evaluate the minimal detectable index resolution $R$ : 
$R=\frac{a}{S}$

Low detection accuracy can present a serious instrument limitation to the sensor's performance resulting in a high minimal detectable index resolution. The detection accuracy for these experiments was $1 \mathrm{pm}$, which could potentially result in a $2 x 10^{-6}$ r.i.u.minimal detectable index resolution. However, the width of the SPR is large $(6 \mathrm{~nm})$ making pm range precision unlikely.

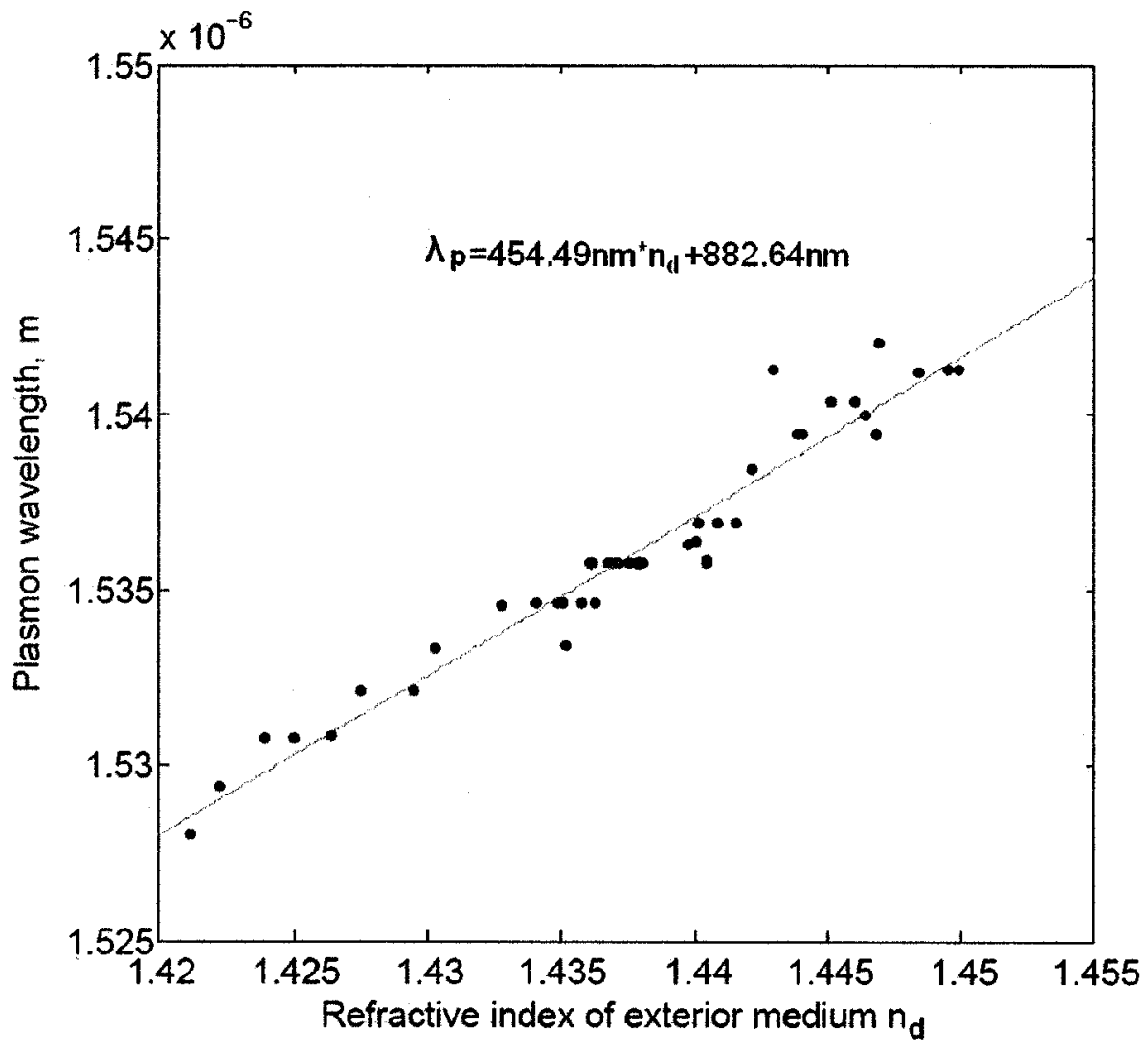

Figure 6.7. Dependence of the plasmon resonance peak wavelength $(\lambda p)$ on the refractive index of the exterior medium at $589 \mathrm{~nm}\left(\mathrm{n}_{\mathrm{d}}\right)$.

\subsubsection{Angle of incidence}

As can be seen from Figure 6.3 the SPR width is approximately $6 \mathrm{~nm}$ and involves usually 10 cladding resonances that are spaced $0.6 \mathrm{~nm}$ apart (Figure 6.8). 
Calculating the angles of incidence $\theta$ of the cladding modes involved, allows the comparison of the performance for the TFBG and prism-based sensors. Each cladding mode resonance appearing in the transmission spectrum can be associated to an angle of incidence $\theta$ which is formed by the reflection of the optical ray at the cladding-metal interface (Figure 4.1). This angle was found through equation 4.3 and plotted versus the transmission loss (Figure 6.9). The angular width of the SPR was determined to be approximately $2.3^{\circ}$ to $3^{\circ}$, which is quite narrow and very close to that reported for SPR in the Kretschmann configuration [Homola, 2003].

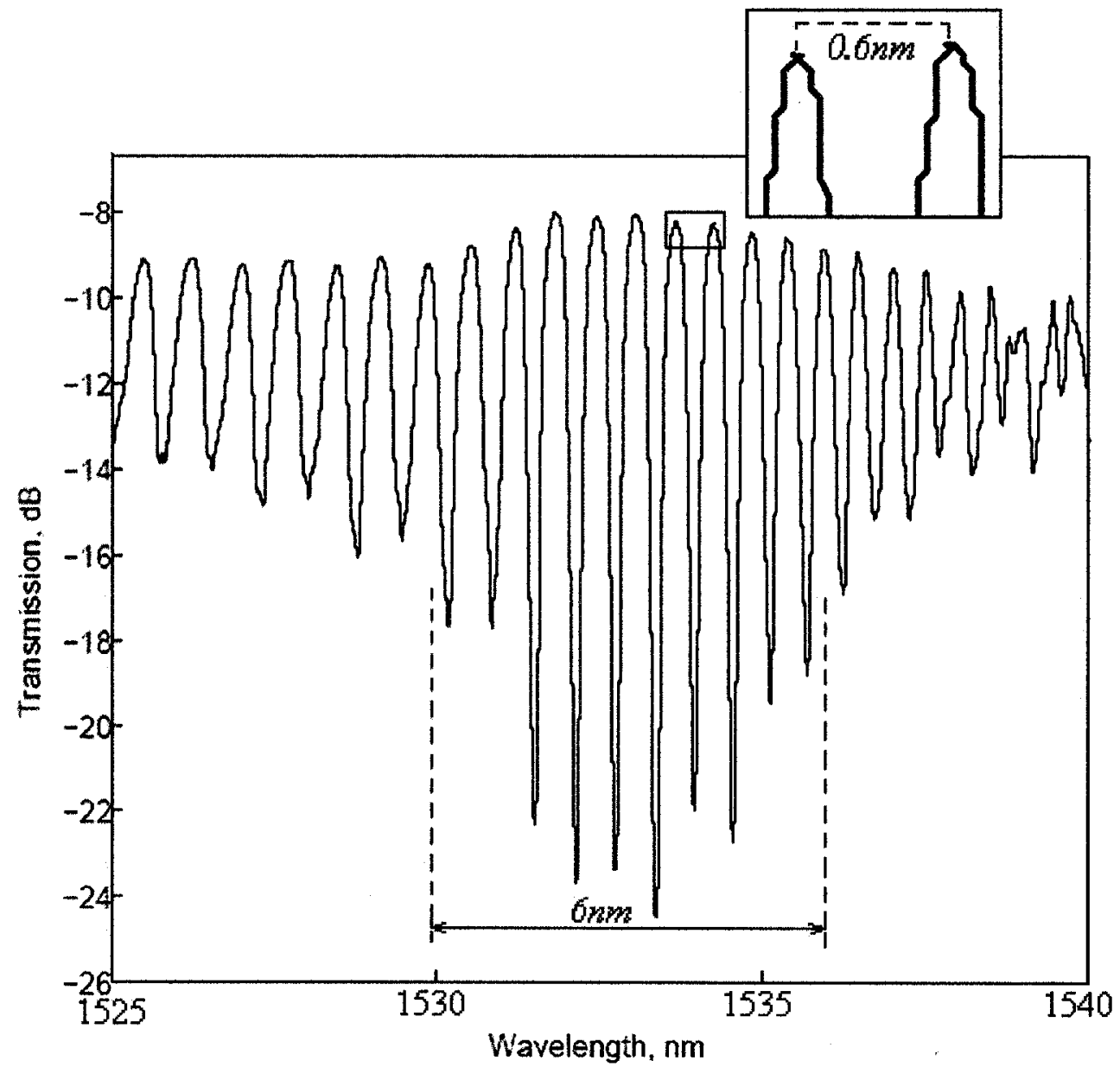

Figure 6.8. Transmission spectrum for $n_{d}=1.4305$. 


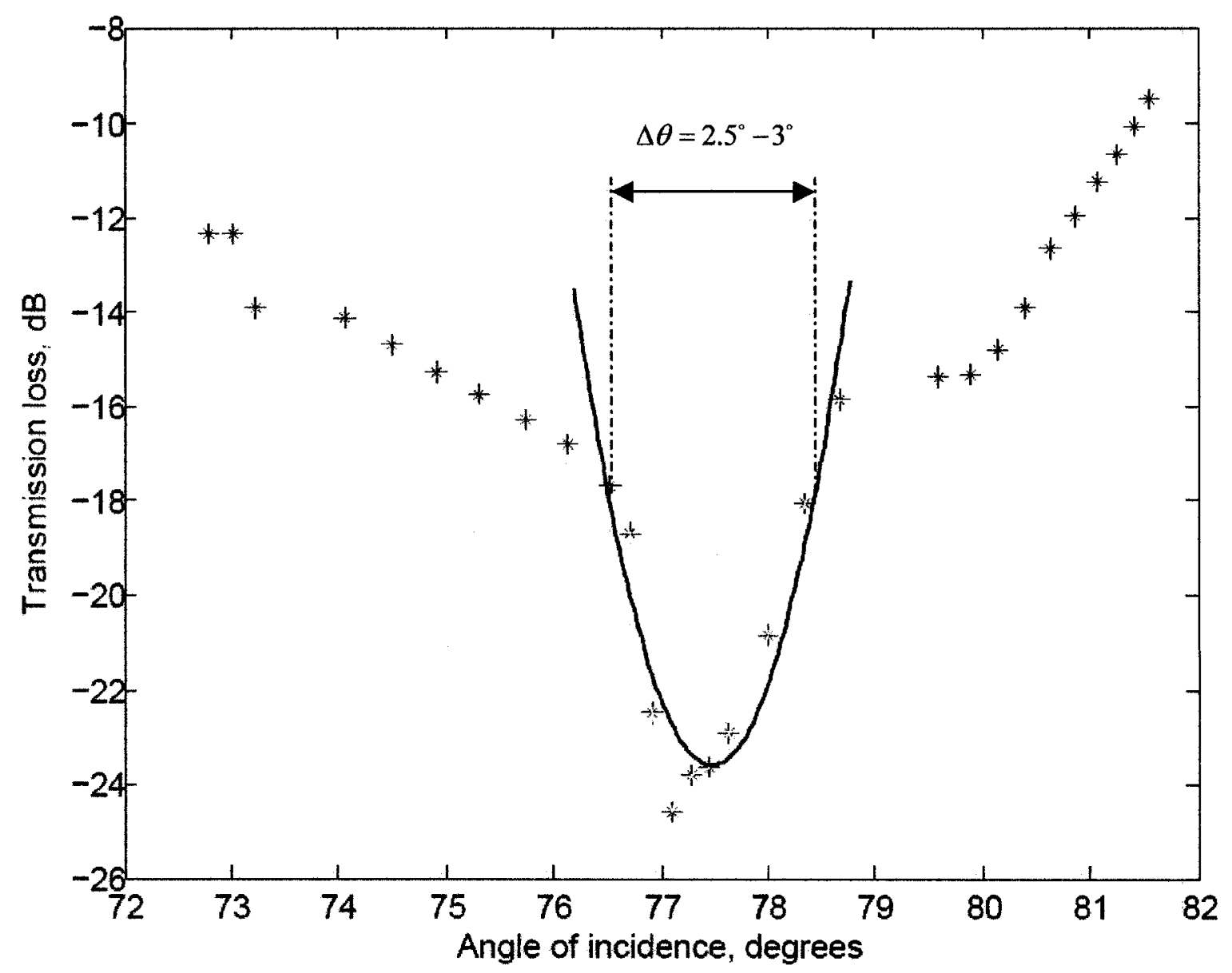

Figure 6.9. Transmission loss versus angle of incidence $\theta$ for $\mathrm{n}_{\mathrm{d}}=1.4378$.

\subsubsection{Gold film characterization}

To find the influence of the gold film parameters on the SPR sensor performance the film characteristics were evaluated with aid of an Atomic Force Microscope (AFM) and Scanning Electron Microscopy (SEM). To characterize the quality of the surface and reliability of the gold layer, SEM images of sensors before and after the experiments with sugar solutions were acquired. Figure 6.10 displays images of the sensor immediately after the gold coating $(20 \mathrm{~nm})$ was deposited (prior to any experiment in solution).

Figure 6.11 shows images of the same fiber after 10 immersions in sugar solutions. It is evident that the gold coating immediately after gold deposition is showing imperfections. An explanation for the imperfections could be a result of the cladding surface not being cleaned properly before gold deposition or by a presence of dust and or 
other noise in the laboratory where the deposition was conducted. Figure 6.11 reveals that the gold surface of fiber is more contaminated and damaged after experiments. Figure 6.12 shows another sensor after being intensively used (about 30 experiments). Its surface is more damaged; the size of the defects became bigger.

The quality of images does allow us to determine the nature of the defects seen on the coating. It was suggested by Professor A. Ianoul (Carleton University, Department of Chemistry) that dark spots on the surface of the film are non-conducting areas, therefore they can be depositions of sugar crystals on the film's surface. However, they could be scratches due to mechanical abrasion occurring during the experiments. Given that the gold layer is quickly degraded after a small number of applications it can be concluded that the sensor can only be used a limited number of times or disposed of after a few experiment. This is not a major issue in many applications because the individual cost of the sensor can be very low in mass production.

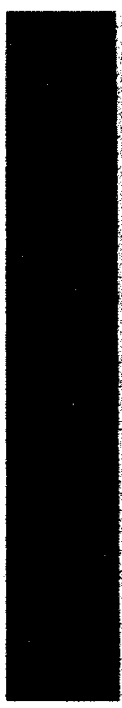

(A)

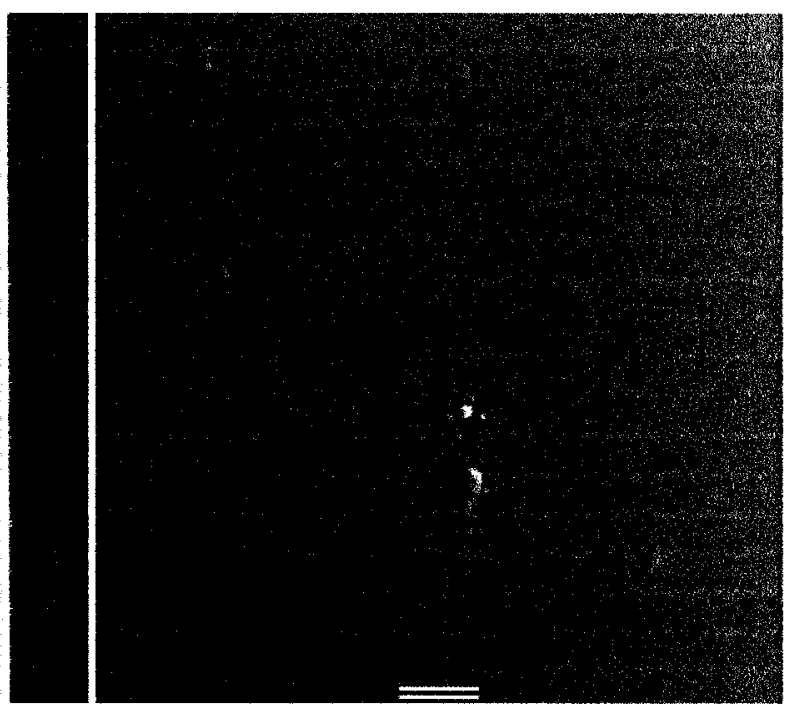

(B)

Figure 6.10. SEM images of TFBG fiber with gold coating (nominal thickness $20 \mathrm{~nm}$ ) before experiments. 

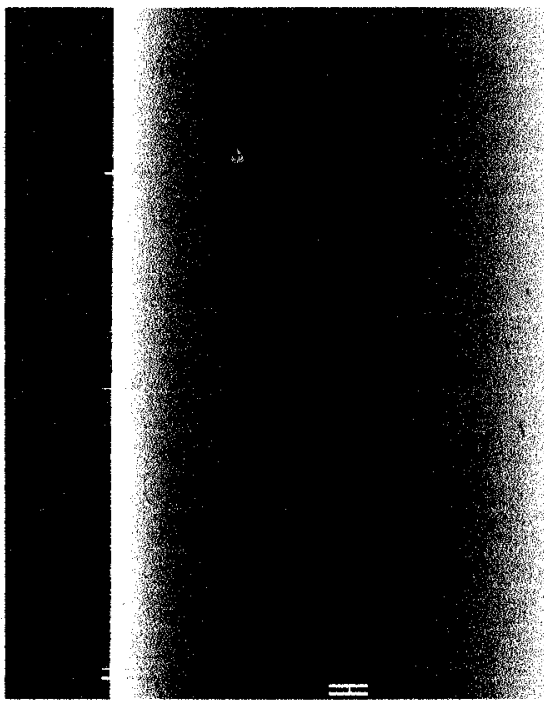

(A)

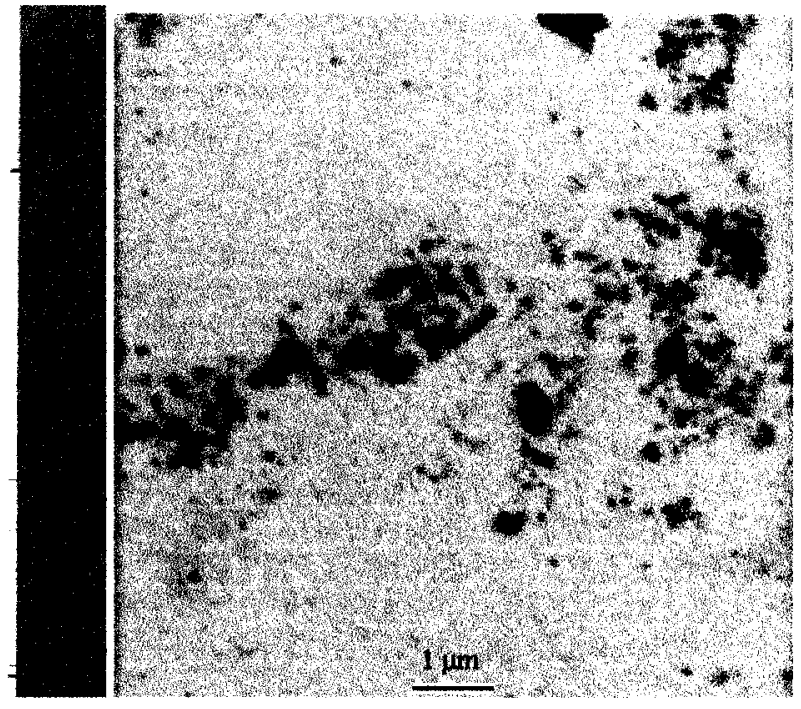

(B)

Figure 6.11. SEM images of TFBG fiber with gold coating $(20 \mathrm{~nm})$ after 10 experiments in sugar solutions [Shevchenko et al., 2007].
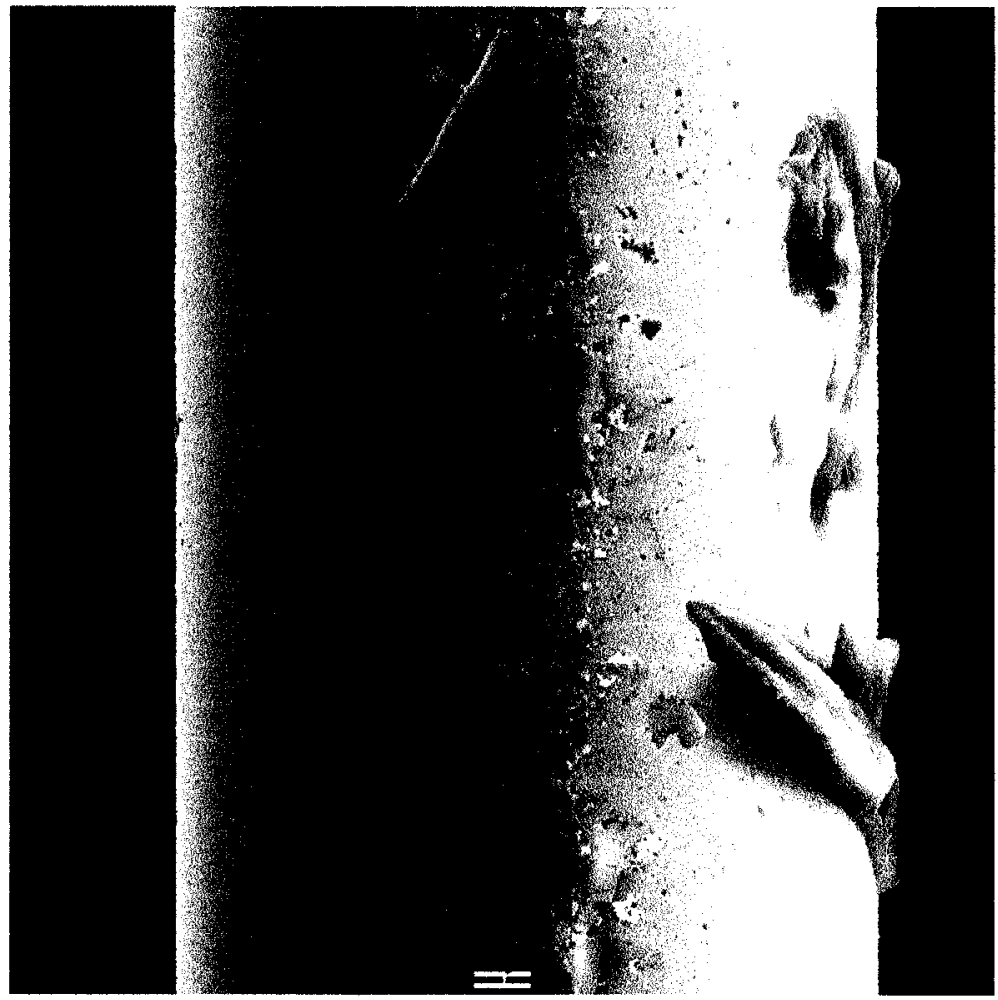

Figure 6.12. SEM images of TFBG fiber with gold coating ( $20 \mathrm{~nm}$ ) after intense testing in sugar solutions (approximately 30 experiments). 
As previously discussed it was quite likely that the gold coating was non-uniform. To measure the thickness an AFM was used to profile the fiber surface at three random locations where the gold film was removed by mechanical abrasion, chemical etching and by laser burning.

The gold film surface profile obtained with mechanical scratching is shown in Figure 6.13, where a high non-uniformity of the gold layer and graininess similar to the defects seen in the SEM images can be observed. The measured values of the gold film thickness in several measurements carried out at several random locations revealed a variance in the thickness between $14.5 \mathrm{~nm}$ to $26.8 \mathrm{~nm}$ (for deposition conditions that yield $20 \mathrm{~nm}$ films on planar substrates). This variance is likely due to variations in overlap between the films obtained during the two depositions carried out on opposite sides of the fiber. The AFM measurements were performed by Professor A. Ianoul in the Steacie Institute for Molecular Sciences, National Research Council (NRC). 

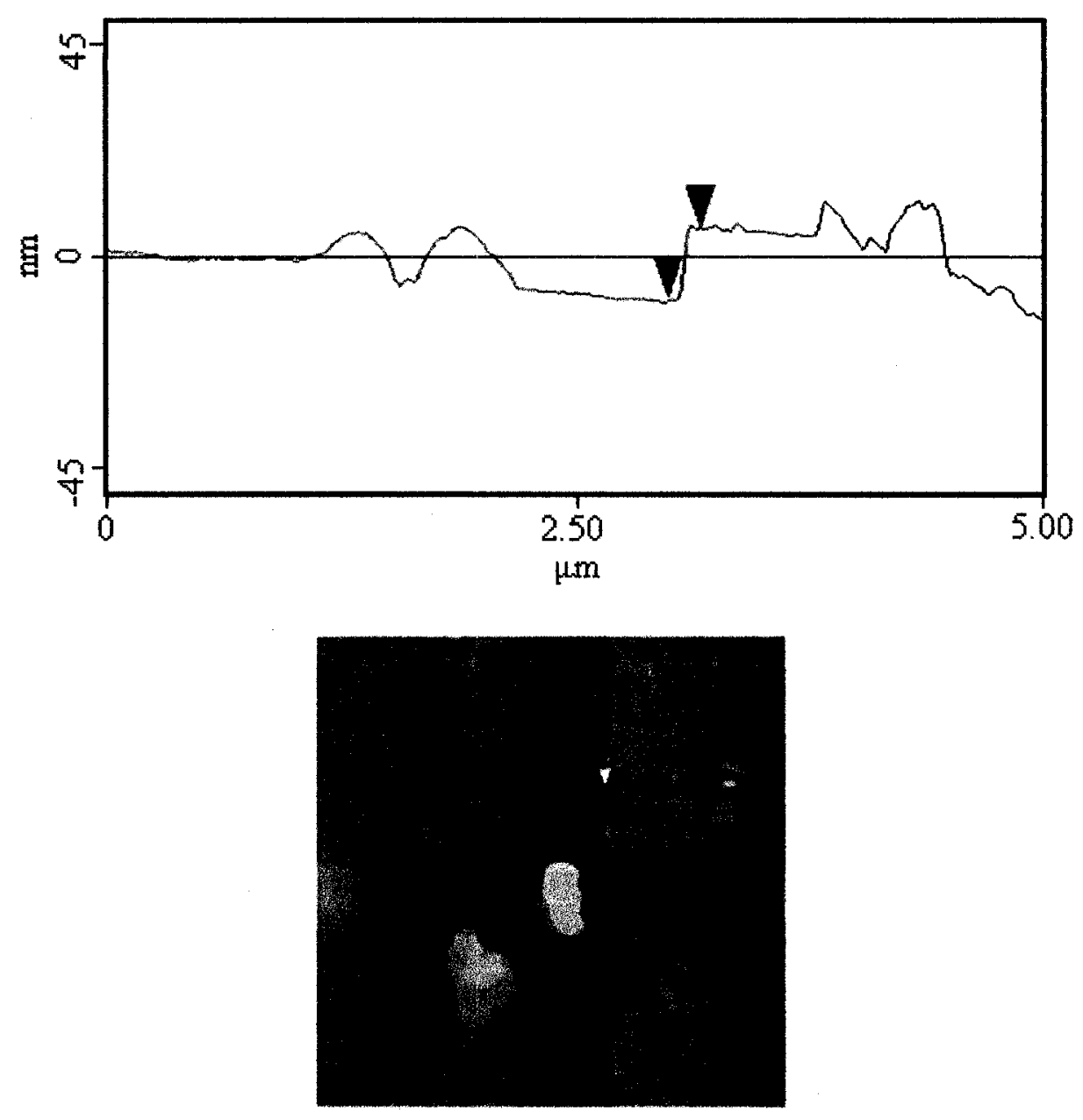

Figure 6.13. AFM Section Analysis of the gold coating near an edge where mechanical abrasion was used to partially remove the coating. The vertical distance between red cursors is $26.783 \mathrm{~nm}$; horizontal distance between red cursors is $218.75 \mathrm{~nm}$ [Shevchenko et al., 2007].

\subsection{Experimental results for sensor with 30 and $50 \mathrm{~nm}$ of the gold layer thickness}

Since the experimental work with the sensor that had a $20 \mathrm{~nm}$ gold layer thickness showed that SPR can be excited on the surface of the gold-coated fiber and SPR was quite a strong effect, the next step was to check if the increase in the gold layer thickness changes the observed phenomena. Results of simulations provided in Section 4.2 .4 showed that optimal gold thickness of the sensor should be between $20 \mathrm{~nm}$ to $65 \mathrm{~nm}$ dependently on the refractive index of the sensed medium. 
New fabricated sensors were coated with $30 \mathrm{~nm}$ and $50 \mathrm{~nm}$ thickness of gold. Results presented in Figures 6.7 and 6.8 show the transmission spectra for these sensors immersed in different sugar solutions. As can be seen from these figures, SPR peaks appear in spectra, however, they are not so strong and pronounced comparing to the 20 $\mathrm{nm}$-coated grating (Figure 6.5). When evaluating the $30 \mathrm{~nm}$ coated grating it is still possible to distinguish SPR-related peaks, in case of $50 \mathrm{~nm}$-coated grating it is less obvious.

Gratings with $30 \mathrm{~nm}$ and $50 \mathrm{~nm}$ of gold were tested in the sugar solutions with similar ranges of refractive index. The $30 \mathrm{~nm}$-coated grating results were similar to the predicted results and to results obtained for the $20 \mathrm{~nm}$-coated grating. The sensitivity was $420.5 \mathrm{~nm} /$ r.i.u. In case of the $50 \mathrm{~nm}$-coated grating it was not always possible to distinguish the SPR peak because the resonance was not strong enough. The obtained sensitivity for this grating was $539.58 \mathrm{~nm} / \mathrm{r}$.i.u, which is very close to the predicted value. 

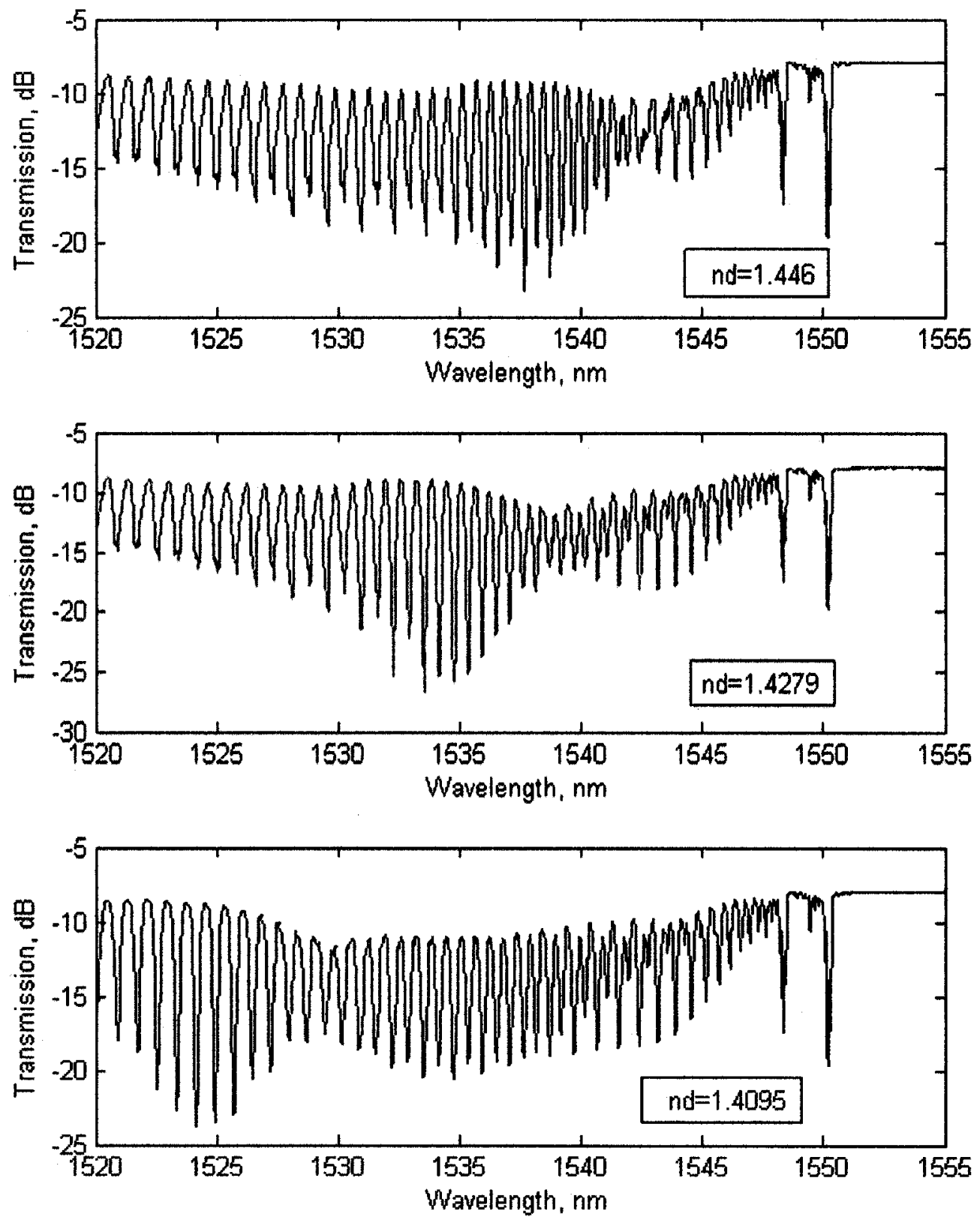

Figure 6.14. Transmission spectra for grating with gold thickness of $30 \mathrm{~nm}$ immersed in different sugar solutions (tilt angle is $6^{\circ}$ ). 

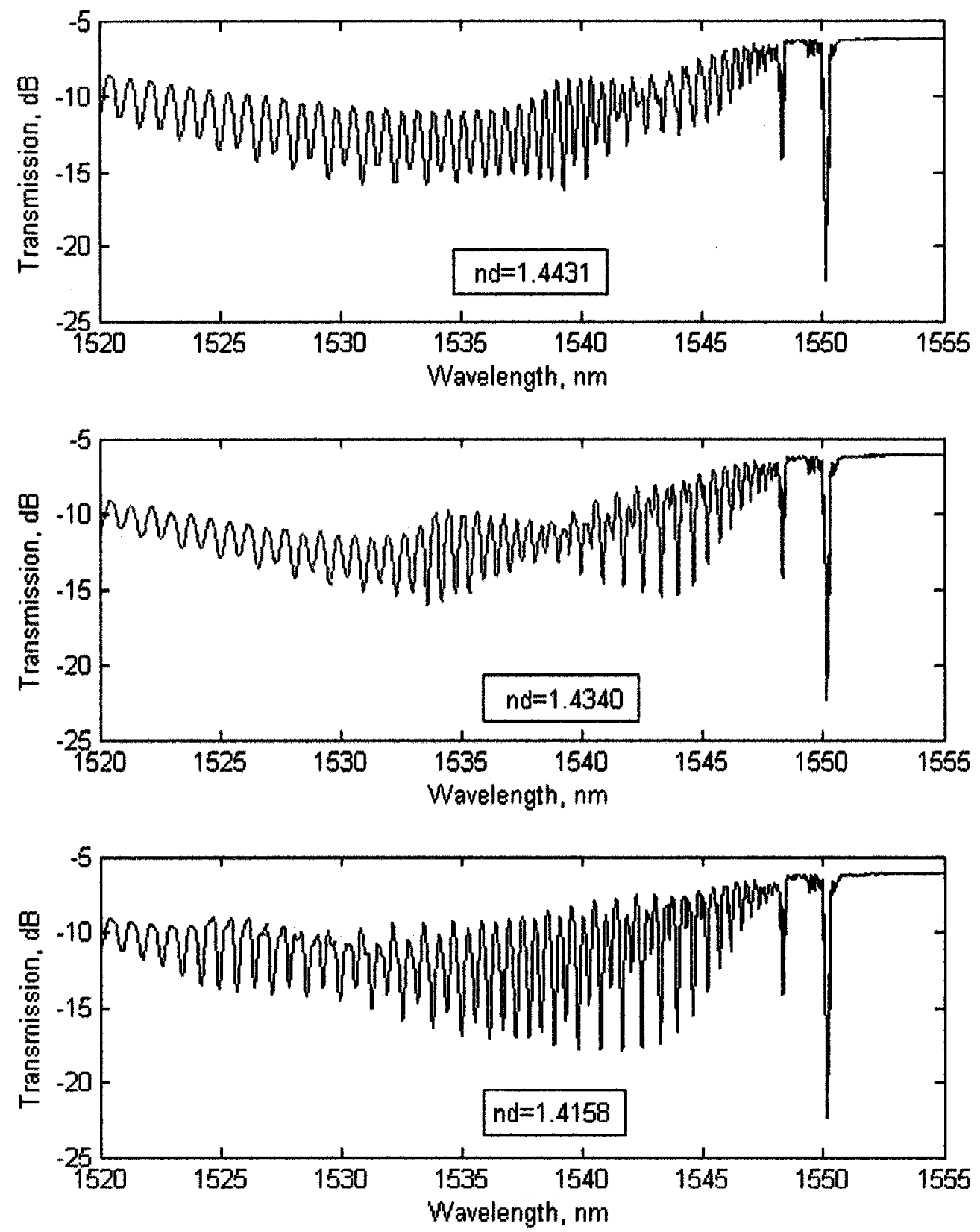

Figure 6.15. Transmission spectra for grating with gold thickness of $50 \mathrm{~nm}$ immersed in different sugar solutions (tilt angle is $6^{\circ}$ ).

According to the calculations, $20 \mathrm{~nm}$ is an optimal gold thickness only for sensing in a small region of refractive index (approximately 1.431). For the rest of the refractive index values within the operating range the optimal gold thickness should be higher than $20 \mathrm{~nm}$ and reaches $65 \mathrm{~nm}$. However, experiments showed that the sensor with $20 \mathrm{~nm}$ of 
metal thickness had the strongest SPR effect over the whole operating range tested. Sensors with $30 \mathrm{~nm}$ and $50 \mathrm{~nm}$ of coating independent of the refractive index of the sensed medium showed a weak and ill-defined SPR peak.

The main conclusion of this latter set of results is that in the cylindrical geometry the optimal metal thickness is smaller than that predicted thickness of the planar model since $50 \mathrm{~nm}$ and $30 \mathrm{~nm}$ of gold appear to be too thick to allow the tunneling of the light across the metal compared to results obtained with $20 \mathrm{~nm}$ gold coating.

\subsection{Discussion}

\section{1) Sensitivity:}

Overall the combined TFBG and SPR theories were applied to the fiber based SPR sensor configuration and successfully predicted the SPR behavior. The SPR effect appeared as an increased attenuation at specific cladding mode resonances in the transmission spectra.

The predicted sensitivity was found to be $543.5 \mathrm{~nm} /$ r.i.u. The experimentally obtained sensitivity was $454,420.5$ and $539 \mathrm{~nm} /$ r.i.u for 20,30 and $50 \mathrm{~nm}$-coated gratings respectively. This difference could not be due to instrument error, but could be possibly explained by an incorrect determination of the SPR wavelength, which could happen as a result of presence of several resonances in the SPR peak. Usually the SPR resonance had 3 or 4 'leading' peaks, which had the largest amplitude. It was almost always possible to determine the strongest peak indicating the wavelength of SPR. However in some cases (as in Figure 6.14 (blue spectrum)) it was more complicated to determine which resonance had the bigger amplitude. Narrowing of the SPR peak (decreasing of the number of cladding modes which resonate together) could possibly increase the accuracy when determining the wavelength of SPR.

Another possible source of errors could be in the whole experimental setup which was not ideal considering that holding the fiber between two slides did not ensure the same physical conditions during each experiment. There could easily be strain and pressure changes each time the fibers were positioned. Additionally, the tails of the fiber that were not fixed under the glass slide could be moved. Strain-related changes in the 
spectrum could result in a wavelength shift of the low-order cladding mode resonances. Such shifts could potentially introduce an error in the SPR wavelength readings.

\section{2) Operating range $\Delta n_{d}$ :}

For the proposed sensor, the predicted operating range $\Delta n_{d}$ for the refractive index of the sensed medium $n_{d}$ which would support SPR at wavelengths $1520.947 \mathrm{~nm}$ to $1546.61 \mathrm{~nm}$ (about 50 cladding modes) was $\Delta n_{d}=0.475$ r.i.u. (1.3967 to 1.4442 ). Due to experimental limitations the sensors were not tested over the complete operating range. The $20 \mathrm{~nm}$-coated grating was tested from 1.4111 to 1.4429 . The $30 \mathrm{~nm}$-coated grating was tested from 1.414 to 1.446 . The $50 \mathrm{~nm}$-coated grating was tested from 1.4213 to $1.443 \mathrm{c} n_{d}$. As previously mentioned the experimentally obtained operating range shifted by 0.026 r.i.u. towards a higher refractive index from the predicted operating range. This shift could possibly be explained by the dispersion of the sugar solution. The refractive index of the sugar solution was measured by an Abbe refractometer, which works at a wavelength $589.3 \mathrm{~nm}$, but the predicted operating range was calculated for the $1520 \mathrm{~nm}$ to $1550 \mathrm{~nm}$ of wavelength region.

The proposed operating range $\Delta n_{d}$ appears wide in comparison to other operating ranges of SPR fiber sensors based on FBGs and LPGs where only one particular mode can be used for SPR excitation. The operating range $\Delta n_{d}$ could also be increased towards a smaller $n_{d}$ value by raising the tilt angle of the grating [Chen et al., 2007].

If in future experiments there is a need for a shift in the operating range towards a lower $n_{d}$ (for example measurement of aqueous mediums), in addition to a larger tilt angle this could be done by depositing a thin dielectric overlay with a high refractive index on the surface of the metal layer [Ctyroky et al., 2007].

\section{3) Surface plasmon resonance peak:}

The width of the SPR is a crucial parameter, it determines how accurate the SPR wavelength position can be measured which in turn determines the accuracy of the SPR sensor. As it was shown in Figure 6.8 the SPR appears as approximately 10 cladding mode resonances with increased amplitude. Each of the resonances is separated from 
neighbor resonances by $0.6 \mathrm{~nm}$. All together these peaks result in $6 \mathrm{~nm}$ (or $3^{\circ}$ ) for the approximate SPR width. This width is much narrower than widths reported for other fiber-based SPR sensors where approximately $100 \mathrm{~nm}$ of width for a single SPR peak is reported in Allsop et al, (2007) and $20 \mathrm{~nm}$ to $40 \mathrm{~nm}$ in Slavik et al, (2001). Despite the fact that the total width of the resonance is better than the results obtained for other fiber sensors and similar to the SPR width of the prism based sensors it still can be potentially narrowed down with the help of two factors.

The first factor is related to the gold quality and uniformity. Deposition of the uniform metal coating around the fiber with precise control of the deposited film and a reduction in the graininess should narrow the plasmon resonance and lead to more precise results.

The second factor is associated with the homogeneity of sensed solution. For this research the sensors were tested in sugar solution that was not evaluated for homogeneity. It is expected that incomplete dissolving of the sugar particles could influence the width of SPR. The average size of the sugar particle is no less than several hundreds of $\mu \mathrm{m}$, which is much larger than the propagation length of SPW. If some of the sugar particles do not dissolve in the solution and approach the gold surface that supports SPR it can seriously change the results in measurement of the refractive index of the solution and lead to ambiguities in the experimental results. Use of the homogeneous solutions such as oils might be beneficial for future work.

\section{4) Gold coating uniformity and surface quality:}

As shown in section 6.2.4 the gold coating is not only non-uniform, but is also unstable and quickly degrades. Figures 6.11 and 6.12 show the gold surface defects that appeared after the fiber was intensively used in the experiments with sugar solutions. Given that the nature of these defects is not obvious from the available images, it is suggested that these defects are deposited sugar crystals. Assuming this suggestion it can be proposed that defects are dielectric and have size $\leq 1 \mu \mathrm{m}$. The refractive index of these formations (if they are sugar crystals) is much higher than the refractive index of any sugar solution. Because of these parameters (size of the defects and their refractive index), each of these defects forms a local section on the gold surface where SPR will not be excited or if SPW is excited, SPW will have a very different propagation constant. 
In case of the first scenario where SPR is not being excited on the surface of the defect, the defect creates an interruption (gap) of the propagation of the plasmon supported by the rest of the gold surface. Recent research shows that SPWs are able to cross the interruptions (in the length up to several $\mu \mathrm{m}$ ), but they lose intensity and may also generate additional SPR modes [Sidorenko and Martin, 2007].

The second scenario creates a local section where a localized SPR effect is created different from the SPR supported by the rest of the fiber. Situation with several defects with different geometrical parameters (as presented in Figure 6.11) could possibly result in an excitement of several SPWs on the surface of the gold-coated fiber with effective indices differing by small amounts from each other. This hypothetically could lead to the widening of the total SPR, which could result in an increase in uncertainty of the experimental results because the SPR wavelength could be determined incorrectly. Misinterpretation of the experimental results caused by widening of SPR could change the sensor's precision.

The non-uniformity of the gold coating caused by the overlap of two gold coatings deposited on two opposite sides of the fiber was not completely investigated. AFM was used to find a metal layer thickness at three random locations along the surface of the fiber where three methods of gold removal were tested (mechanical scratching, laser burning and chemical etching). The next step in this research should involve a determination of the deposited gold thickness at equally spaced intervals around the circumference of the fiber which is required to understand the relation between gold thickness and SPW (and their number). Recent research in this field showed that deposition of two semi-cylindrical coatings (one on every side of fiber) allows excitation of several different hybrid surface modes at specific values of $n_{d}$ [Monzon-Hernandez and Villatoro, 2006]. Results provided by Monzon-Hernandez and Lillatoro, (2006) resemble the spectrum in Figure 6.6. Excitation of several SPR modes with different effective indices could explain this phenomenon, however this requires further investigation employing a theoretical model.

\section{5) Gold thickness}

Absorption-based SPR theory was used to find the optimal metal layer thickness for SPR excitement. For values of the refractive index of the exterior medium varying 
from 1.36 to 1.5 the optimal gold thickness was in the range $20 \mathrm{~nm}$ to $120 \mathrm{~nm}$. Experimental results reveal that plasmon effect has the strongest amplitude for $20 \mathrm{~nm}$ of gold coating and decreases as the thickness of metal increases. A gold thickness of $50 \mathrm{~nm}$ showed the weakest SPR, which was difficult to distinguish at low refractive indices of the sensed medium. Discrepancies between experimental results and simulations are difficult to explain based solely on the theory developed for the prism configuration. In order to achieve a better understanding of this phenomenon further experimental work is required to test the effects of different gold layer thickness and by adjusting absorptionbased SPR theory for fiber-based sensors. 


\section{Chapter 7. Conclusion and Future Work Recommendations}

\subsection{Conclusion}

The main goal of this thesis was to propose a new SPR fiber-based sensor. A review of the literature revealed that a variety of SPR fiber-based sensors have been proposed, but many lack simplicity, are fragile due to the introduced fiber deformations and use of non-standard optical fibers. The SPR effect in many of them is very wide and poorly defined. Employing fiber gratings and eliminating the need for fiber deformations can overcome these drawbacks. The sensor presented in this thesis not only has dealt with these problems, but has also an improved sensitivity, simpler manufacturing requirements and is considered to be more cost effective.

The sensor is based on a standard single-mode fiber (Corning SMF28) with a TFBG imprinted in its core and a gold coating on the fiber's surface. The TFBG component with a small tilt angle is considered a key element in the sensor's design making it possible to excite the SPRs. The TFBG couples the forward propagating core mode to the set of back-propagating cladding modes. These cladding modes propagate in proximity to the cladding-metal interface and by refracting at this interface generate evanescent fields penetrating into the metal layer. If one of the evanescent fields has propagating constant that matches the propagation constant of a SPW, a SPR is excited on the outer gold layer surface.

Before the experiment was conducted, the sensor's performance was characterized using the TFBG matching condition and SPR theory developed for the prism-based configuration with planar geometry. The operating range, sensitivity and optimal metal layer thickness were calculated.

The fabrication of the sensor is fairly simple and fully described in Chapter 5. A standard single-mode fiber was used as the sensor's base. Fabrication of the tilted grating required several steps that included cleaning of the fiber, hydrogen loading and writing of the grating followed by an annealing in the oven. In the conducted experiments, the gratings were written with a side-writing technique, where an interferometric pattern was formed using a phase mask and projected on the side of the fiber. 
Gold was selected among possible SPR “active" metals for its ability to form different transducing layers on its surface via different immobilizing techniques. Deposition of the gold was done in a sputtering chamber via vacuum deposition. This deposition process resulted in a non-uniform coating that was later.

Experimental results demonstrated the possibility to excite SPR with the proposed sensor with good correlation between the predicted and experimentally obtained sensor parameters. The sensor's sensitivity almost matched the predicted one. The SPR peak width is one of the narrowest reported to date. The operating range of the sensor was shifted towards a higher refractive index; however it could be explained by the sugar solution dispersion, which was not taken into account during the calculations.

Gold thickness was found to be an important parameter influencing the sensor's performance. Results show a strong SPR effect for $20 \mathrm{~nm}$ of coating followed by a decay of the SPR effect as the gold thickness increased to $50 \mathrm{~nm}$. This result is opposite to the results obtained by the absorption-based SPR theory developed for the planar geometry. This discrepancy should be investigated in future experiments in order to optimize the sensor's performance.

Overall this thesis showed that a combination of both TFBG technology and the SPR effect resulted in a high-performance sensor where high sensitivity is accomplished by such valuable properties as simplicity, accuracy and cost-effectiveness. Our initial experimental demonstration of this novel sensor was published recently in Optics Letters [Shevchenko and Albert, 2006].

\subsection{Future work outline}

After analyzing the experimental results and summarizing the main ideas discussed in the previous chapters it is possible to outline several trends for which future work in this area is suggested.

\section{1) Narrowing down the SPR and increasing the precision of sensor.}

This can be done by using a more homogenous sensed medium (for example oils) and by depositing a uniform metal layer with better surface quality. Improvement in surface quality can be achieved through use of such depositing techniques as Atomic Layer deposition or Gold Plating (both are currently being investigated). 
Repetition of the experiments using the same conditions is crucial for determining the sensor's performance. In order to achieve good repeatability of the experiments and eliminate random sources of noise such as pressure changes between the glass slide, and bending of different parts of fiber, a new experimental setup should be established.

SPR is very sensitive to light polarization; it is known that it can be excited only with TM waves. Although the light used in this research had uncontrolled polarization it is recommended to try different polarizations for SPR tuning in future work. This may increase coupling strength between the cladding modes and SPW leading to better accuracies.

\section{2) Further sensor optimizations}

Given that the proposed sensor is able to excite SPR on its surface the next step will be to optimize the design of the sensor. This includes not only narrowing SPR, but also finding the optimal gold thickness. This can be accomplished by performing more experimental tests on the sensor with different thickness and by additional simulations.

An increase in the understanding of the SPR effect may also provide insight into optimization methods. For example, experiments showed that in some cases SPR peaks were changing their amplitudes. While some were decreasing, others were increasing however their wavelength positions were not changing. It would be great to understand these processes and employ them to expand sensing possibilities.

\section{3) Investigating cross-sensitivity}

Uncoated TFBGs are sensitive to strain, temperature and the refractive index of exterior. For instance, in chemical sensors based on uncoated TFBG, the evanescent fields of cladding modes are able to reach the exterior and interact with it. These interactions introduce wavelength shifts for high order cladding modes. Depositing of the metal layer on TFBG and excitation of SPR does not cancel the interaction of evanescent fields with the exterior, however it may change the interaction amplitude. In the same way strain related-shifts occurring for low-order cladding modes may interact with 
plasmon-related spectrum changes. All these aspects should be explored to help enhance the sensors capabilities and help determine possible applications.

\section{4) Develop sensor for specific applications}

Gold provides great opportunity to create thin organic and non-organic films on its surface via different immobilization chemistries. Usually SPR penetration in the sensed medium is very small (about several hundreds of $\mathrm{nm}$ ) [Ligler and Taitt, 2002]. One of the most attractive perspectives is to try immobilizing certain ligands on the gold surface with and uses the sensor to characterize the interaction of this ligand with specific targets. It would allow enable real-time measurements of the refractive index and thickness of organized layers on the gold's surface, possibly in situ because of the very small size of the sensor device itself and the integrated data transmission medium: the remainder of the optical fiber. Because we are using standard fiber in its low loss window $(1550 \mathrm{~nm})$ our sensor could be integrated up to several $\mathrm{km}$ away without difficulty. 


\section{References}

Allsop T., Neal R., Rehman S., Webb D.J., Mapps D., and Bennion I., "Generation of Infrared Surface Plasmon Resonances with High Refractive Index Sensitivity Utilizing Tilted Fiber Bragg Gratings, Applied Optics, vol. 46, no. 22, 2007, pp. 5456 - 5460.

Autolab, "SPR Instrumentation" [online], Autolab, Eco Chemie, [cited July 1, 2007], available from World Wide Web:

http://www.ecochemie.nl/?pag=8

Barnes W.L., Dereux A., and Ebbesen T.W., "Surface Plasmon Subwavelength Optics," Nature, vol. 424, 2003, pp. 824-830.

Baron, A., "Biocore Surface Plasmon Resonance (SPR)" [online], Astbury Centre for Molecular Biology, 2004 [cited July 1, 2007], available from World Wide Web: http://www.astbury.leeds.ac.uk/facil/SPR/spr_intro2004.htm

Battiato J.M., "Fiber Bragg Gratings," $\mathrm{PhD}$ thesis, Faculty of Optical Sciences, University of Arizona, Arizona, USA, 1998.

Berini P., "Plasmon-Polariton Waves Guided by Thin Lossy Metal Films of Finite Width: Bound Modes of Symmetric Structures," Physical Review B, vol. 61, 2000, pp. 10484-10503.

Berini P., "Plasmon-Polariton Waves Guided by Thin Lossy Metal Films of Finite Width: Bound Modes of Asymmetric Structures," Physical Review B, vol. 61, no. 15, 2001, pp. 10484-10503.

Berger N.K., Levit B., Fischer B., Kulishov M., Plant D.V., and Azaña J., "Temporal Differentiation of Optical Signals Using a Phase-Shifted Fiber Bragg Grating," Optics Express, vol. 15, no. 2, 2007, pp. 371-381. 
Blakemore J.S., Solid State Physics, 2-end ed., Cambridge University Press, 1985.

Boardman A.D., Electromagnetic Surface Modes, Wiley, New York, 1982.

Boltasseva A., Bozhevolnyi S. I., Nikolajsen T., and Leosson K., "Compact Bragg Gratings for Long-Range Surface Plasmon Polaritons," Journal of Lightwave Technology, vol. 24, no. 2, 2006, pp. 912-918.

Buric M., Chen K.P., Bhattarai M., Swinehart P.R., and Maklad M., "Active Fiber Bragg Grating Hydrogen Sensors for All-Temperature Operation", IEEE Photonics Technology Letters, vol.19, no.5, 2007, pp.255-257.

Burke J.J., Stegemen G.I., and Tamir T., "Surface-Polariton-Like Waves Guided by Thin Lossy Metal Films," Physical Review B, vol. 33, no. 8, 1986, pp. 5186-5201.

Chen C., and Albert J., "Strain-Optic Coefficients of the Individual Cladding Modes of a Single Mode Fiber: Theory and Experiment," Electronics Letters, vol. 48, no. 7, 2006, pp. 1027-1028.

Chen C., Shevchenko Y.Y., and Albert J., 'Novel Sensing Mechanisms using Tilted Fiber Bragg Gratings', in Optical Waveguide Sensing and Imaging, W. Bock, I. Gannot, and S. Tanev (ed), Springer, to be published, 2007.

Chinowsky T.M., Quinn J.G., Bartholomew D.U., Keiser R., and Elkind J.L., "Performance of the Spreeta 2000 Integrated Surface Plasmon Resonance Affinity Sensor" [online], Texas Instruments Incorporated, [cited July 1, 2007], available from World Wide Web:

http://focus.ti.com/general/docs/gencontent.tsp?contentId=29566 
Corning Incorporated, “Corning SMF-28 Optical Fiber Product Information" [online]

[cited August 25, 2007], available from World Wide Web:

http://www.ee.byu.edu/photonics/connectors.parts/smf28.pdf

Cusano A., Persiano G.V., Russo M., and Giordano M., "Novel Optoelectronic Sensing System for Thin Polymer Films Glass Transition Investigation," IEEE Sensors Journal, vol. 4 , no. 6,2004 , pp. 837-844.

Ctyroky J., Homola J., and Skalsk M., "Tuning of Spectral Operation Range of a Waveguide Surface Plasmon Resonance Sensor," Electronics Letters, vol. 33, no. 14, 1997, pp. 1246-1248.

De Young R.J., "A Narrow Band Fiber Bragg Grating Filter for LIDAR Receivers," in Lasers and Electro-Optics, CLEO '01, May 6-11, 2001, pp. 494-495.

Endo T., Kerman K., Nagatani N., Morita Y., Takamura Y., and Tamiya E., "Localized Surface Plasmon Resonance Based Label-Free Optical Biosensor for Monitoring Peptide Nucleic Acid-DNA Hybridization," Proceedings of 2005 5th IEEE Conference on Nanotechnology, Japan, July 2005.

Erdogan T., "Fiber Grating Spectra," Journal of Lightwave Technology, vol. 15, no. 8, 1997, pp. 1277-1294.

Esteban Ó., Alonso R., Navarrete M.C., and Cano A., "Surface Plasmon Excitation in Fiber-Optics Sensors:A Novel Theoretical Approach," Journal of Lightwave Technology, vol. 20, no. 3, 2002, pp. 448-453.

Esteban Ó., Cano A., Herrera N., and Navarrete M.C., "Absorption as a Selective Mechanism in Surface Plasmon Resonance Fiber Optic Sensors," Optics Letters, vol. 31, no. 21, 2006, pp. 3089-3091. 
Etchegoin P.G., Le Ru E.C., and Meyer M., "An Analytic Model for the Optical Properties of Gold," Journal of Chemical Physics, vol. 125, 2006, pp. 164705.

Fano U., "The Theory of Anomalous Diffraction Gratings and of Quasi-Stationary Waves on Metallic Surfaces (Sommerfeld's waves)," Journal of the Optical Society of America, vol. 31, 1941, pp. 213.

Fernandez A., Brichard B., Berghmans F., Rabii H.E., Fokine M., and Popov M., "Chemical Composition Fiber Gratings in a High Mixed Gamma Neutron Radiation Field," IEEE Transactions on Nuclear Science, vol. 53, no. 3, 2006, pp. 1607-1663.

González-Cano A., Bueno F.-J., Esteban Ó., Díaz-Herrera N., and Navarrete M.-C, "Multiple Surface-Plasmon Resonance in Uniform-Waist Tapered Optical Fibers with an Asymmetric Double-Layer Deposition," Applied Optics, vol. 44, no. 4, 2005, pp. 519526.

Grattan K.T.V., and Meggitt B.T., Optical Fiber Sensor Technology: Advanced Applications - Bragg Grating and Distributed Sensors. Kluwer, Academic Publishers, Boston, USA, 2000.

He Y. -J., Lo Y. -L., and Huang J. -F. “Optical-Fiber Surface-Plasmon-Resonance Sensor Employing Long-Period Fiber Gratings in Multiplexing," Journal of the Optical Society of America B., vol. 23, no. 5, 2006, pp. 801-811.

Hill K.O., "Aperiodic Distributed-Parameter Waveguides for Integrated Optics," Applied Optics, vol. 13, 1973, pp. 1853-1856.

Hill K.O., Fujii Y., Johnson D.C., and Kawasaki B.S., "Photosensitivity in Optical fiber Waveguides: Applications to Reflection Filter Fabrication," Applied Physics Letters, vol. 32 , no. 10,1978 , pp. $647-649$. 
Homola J., "Optical Fiber Sensor Based on Surface Plasmon Excitation," Sensors and Actuators B, vol. 29, 1995, pp. 401-405.

Homola J., "Present and Future of Surface Plasmon Resonance Biosensors," Analytical and Bioanalytical Chemistry, vol. 377, no. 3, 2003, pp. 528-539.

Homola J., Surface Plasmon Resonance Based Sensors. In "Spinger Series on Chemical Sensors and Biosensors. Methods and Applications," O.S.Wolfbeis (ed), Springer-Verlag Berlin Heidelberg, 2006.

Homola J., and Slavik R., "Fibre-Optic Sensor Based on Surface Plasmon Resonance," Electronics Letters, vol. 32, no.5, 1996, pp. 480-482.

Hong-Yu L., Woo-Hu T., Yu-Chia T., and Bor-Chiou S., "Side-Polished Multimode Fiber Biosensor Based on Surface Plasmon Resonance with Halogen Light," Applied Optics, vol. 46, no. 5, 2007, pp. 800-806.

Hosono H., and Abe Y., "Nature and Origin of the 5-eV band in $\mathrm{SiO}_{2}$ : $\mathrm{GeO}_{2}$ Glasses," Physical Review B, vol. 48, no. 18, 1992, pp. 11445-11451.

Hsiesh B. -Y., Chang Y. -F., Ng M. -Y., Liu W. -C., Lin C. -H., Wu H. -T., and Chou C., "Localized Surface Plasmon Coupled Fluorescence Fiber-Optic Biosensor with Gold nanoparticles," Analytical Chemistry, vol. 79, no. 9, 2007, pp. 3487-3493.

Iadicicco A., Cusano A., Campopiano S., Cutolo A., and Giordano M., "Thinned Fiber Bragg Gratings as Refractive Index Sensors," IEEE Sensors Journal, vol. 5, no. 6, 2005, pp. 1288-1295.

IBIS Technologies, "SPR Sensors" [online] IBIS Technologies, Sept. 2006 [cited July 1, 2007], available from World Wide Web:

http://www.ibis-spr.nl/index_2.html 
Ibsen M., Nilsson J., Fu L., Jeong Y., Sahu J. K., Mokhtar M.R., Alegria C. and Payne D.N., "All-Fibre Bragg Grating Filters and Lasers for Future Optical Networks," in Information, Communications and Signal Processing, 2003 and the Fourth Pacific Rim Conference on Multimedia, December 15-18, pp. 178-179.

Ince R., and Narayanaswamy R., "Analysis of the Performance of Interferometry Surface Plasmon Resonance and Luminescence as Biosensors and Chemosensors," Analytica Chimica Acta, vol. 569, no.1-2, 2006, pp. 1-20.

Jafari A., "Tilted Fiber Grating Refractive Index Sensor and Interrogation Device," Masters Thesis, Dept. of Electronics, University of Carleton, Ontario, Canada, May 30 2006.

Jorgenson R.C., and Yee S.S., "A Fiber-Optic Chemical Sensor Based on Surface Plasmon Resonance," Sensors and Actuators, vol. 8, no. 12, 1993, pp. 213-220.

James, S.W. and Tatam R.P., "Optical Fiber Long-Period Grating Sensors: Characteristics and Application." Measurements Science and Technology, vol. 14, 2003, pp. R49-61.

Johnson P.B., and Christy R.W., "Optical constants of the noble metals," Physical Review $B$, vol. 15, no. 12, 1972, pp. 4370-4379.

Kashyap R., Fiber Bragg gratings, Academic Press, San-Diego, California, USA, 1999.

Komukai T., and Nakazawa M., "Long-Phase Error-Free Fiber Bragg Gratings", IEEE Photonics Technology Letters, vol. 10, no. 5, 1998, pp. 687-689. 
Kurihara K., and Suzuki K., "Theoretical Understanding of an Absorption-Based Surface Plasmon Resonance Sensor Based on Kretchmann's Theory," Analytical Chemistry, vol. 74, 2002, pp. 696-701.

Kretschmann, E. and Raether H., "Radioactive Decay of Non-Radioactive Surface. Plasmons Excited by Light," Z. Naturforsch, vol. 23A, 1968, pp. 2135-2136.

Laffont G., and Ferdinand P., "Tilted Short-Period Fiber-Bragg-Grating Induced Coupling to Cladding Modes for Accurate Refractometry," Measurement Science and Technology, vol. 12, 2001, pp. 765-770.

Lemaire P.J., Atkins R.M., Mizrahi V., and Reed W.A., "High-Pressure H2 Loading as a Technique for Achieving Ultrahigh UV Photosensitivity and Thermal Sensitivity in GeO2 Doped Optical Fibers," Electronics letters, vol.29, no. 13, 1993, pp.1191-119

Ligler F.S. and Taitt C.A.R., Optical Biosensors: Present and Future. Elsevier Science, 2002.

Limberger H.G., Fonjallaz P.Y., and Salathe R.P., "Spectral Characterization of Photoinduced High Efficient Bragg Bratings in Standard Telecommunication Fibers," Electronic Letters, vol. 29, no. 1, 1993, pp 47-49.

Lin G. C., Wang L., Yang C. C., Shih M. C., and Chuang T. J., "Thermal Performance of Metal-Clad Fiber Bragg Grating Sensors," IEEE Photonics Technology Letters, vol. 10, no. 3,1998 , pp. $406-408$.

Loh W. H., Laming R. I., Robinson N., Cavaciuti A., Vaninetti F., Anderson C. J., Zervas M. N., and Cole M. J., "Dispersion Compensation Over Distances in Excess of $500 \mathrm{~km}$ for $1 \mathrm{O}-\mathrm{Gb} / \mathrm{s}$ Systems Using Chirped Fiber Gratings," IEEE Photonics Technology Letters, vol. 8, no. 7, 1996, pp. 944-946. 
Loo C., Lowert A., Hala N., West J., and Drezek R., "Immunotargeted Nanoshells for Integrated Cancer Imaging and Therapy," Nano Letters, vol. 5, no. 4, 2005, pp. 709-711.

Malitson, I.H., "Interspecimen Comparison of the Refractive Index of Fused Silica," Journal of the Optical Society of America, vol. 55, no. 10, 1965, pp. 1205-1209.

Malo B., Therialt S.,.Johnson D.C, Bilodeau F., Albert J., and Hill K.O., "Apodised inFibre Bragg Grating Reflectors Photoimprinted Using a Phase Mask," Electronics Letters, vol. 31, no. 3, 1995, pp. 223-225.

Martinez A., Lai Y., Dubov M., Khrushchev I.Y. and Bennion I., "Vector Bending Sensors Based on Fibre Bragg Gratings Inscribed by Infrared Femtosecond Laser," Electronics Letters, vol. 41, no. 8, 2005, pp. 472-474.

Matsuhara M., and Hill K.O., "Optical-Waveguide Band-Rejection Filters: Design," Applied Optics, vol. 13, 1974, pp. 288.

Meltz G., Morey W.W., and Glenn W.H., "Formation of Bragg Gratings in Optical Fibers by a Transverse Holographic Method," Optics Letters, vol. 14, no. 15, 1989, pp. 823-825.

Meltz G., Morey W.W., and Dunphy J.R., "Fiber Bragg Grating Chemical Sensor," in SPIE Chemical, Biochemical, and Environmental Fiber Sensors III, vol. 1587, 1991, pp. 350-361.

Meltz G., Hewlett S.J., and Love J.D., "Fiber Grating Evanescent-Wave Sensors," in SPIE Chemical, Biochemical, and Environmental Fiber Sensors III, vol. 2836, 1996, pp. $342-350$. 
Monzon-Hernandez D., and Villatoro J., "High-Resolution Refractive Index Sensing by Means of a Multiple-Peak Surface Plasmon Resonance Optical Fiber Sensor," Sensors and Actuators $B$, vol. 115 , no. 1, 2006, pp. 227-231.

Nemova G. and Kashyap R. "Fiber-Bragg-Grating-Assisted Surface Plasmon-Polariton Sensor," Optics Letters, vol. 31, no. 14, 2006, pp. 2118-2020.

Nemova G. and Kashyap R., "Modeling of Plasmon-Polariton Refractive-Index Hollow Core Fiber Sensors Assisted by a Fiber Bragg Grating," Journal of Lightwave Technology, vol. 24, no. 10, 2006, pp. 3789-3796.

Okamoto K., Fundamentals of Optical Waveguides.CA, USA: Academic Press, 2000.

O’Neal D.P., Hirsch L.R., Halas N.J., Payne J.D., West J.L., "Photo-Thermal Tumor Ablation in Mice Using Near Infrared-Absorbing Nanoparticles," Cancer Letters, vol. 204, 2004, pp. 171-176.

Othonos A., and Kalli K., Fiber Bragg Gratings: Fundamental and Applications in Telecommunications and Sensing. Artech House, Boston, USA, 1999.

Patrick H. J., Kersey A. D., and Bucholtz F., "Analysis of the Response of Long Period Fiber Gratings to External Index of Refraction," Journal of Lightwave Technology, vol. 16 , no. 9,1998 , pp. 1606-1612.

Patskovsky S., Kabashin A.V., and Meunier M., "Properties and Sensing Characteristics of Surface Plasmon Resonance in Infrared Light," Journal of Optical Society of America, vol. 20 , no. 8, 2203, pp. 1644-1650.

Patskovsky S., Bah S., Meunier M. and Kabashin A. V., "Characterization of High Refractive Index Semiconductor Films by Surface Plasmon Resonance," Applied Optics, vol. 45 , no. 25,2006 , pp. $6640-6646$. 
Piliarik M., Homola J., Manı'kova Z., and Styroky J. C., "Surface Plasmon Resonance Sensor Based on a Single-Mode Polarization Maintaining Optical Fiber," Sensors and Actuators B, vol. 90, 2003, pp. 236-242.

Pockrand I., Swalen J.D., Gordon J.G., Philpott M.R., "Surface Plasmon Spectroscopy of Organic Monolayer Assemblies," Surf Science, vol. 74, no. 1, 1978, pp. 237-244.

Psaila D.C. and H.G. Inglis, "Packaging of Optical Fibre Bragg Gratings," in Electronic Components and Technology Conference Proceedings, pp. 439-443.

Raether H., Surface Plasmons on Smooth and Rough Surfaces and on Gratings. Germany: Springer-Verlag, 1988.

Reichert, "Reichert Surface Plasmon Resonance Instruments" [online] Reichert, Inc., [cited July 1, 2007], available from World Wide Web:

http://www.reichertspr.com/

Rice T., Duncan R., Gifford D., Cooper K., and Furrow K., "Fiber Optic Distributed Strain, Acoustic Emission, and Moisture Detection Sensors for Health Maintenance," in IEEE Systems Readiness Technology Conference Proceedings, September 22-25, 2003, pp. 505-514.

Thiele H., Gimkiewicz C., Gale M. T., Soechtig J., Westenhoefer S., Mahmud S., Zschokke C., "Tandem Chirped Grating Couplers for Data Telecom Monitoring Applications," in Society of Photo-Optical Instrumentation Engineers 2003, vol.2, no. 4, pp. 283-291.

Schewe P., and Stein B., "Plasmon Assisted Solar Cell" [online] Physics News Updates, 2007 [cited August 12, 2007], available form the World Wide Web: http://www.aip.org/pnu/2007/split/819-2.html 
Sharma A. K., and Gupta B.D., "Fiber-Optic Sensor Based on surface Plasmon Resonance with Ag-Au Alloy Nanoparticle Films," Nanotechnology, vol. 17, 2006, pp. 124-131.

Sharma A. K., and Gupta B.D., "Fiber-Optic Sensor Based on Surface Plasmon Resonance with Nanoparticle Films," Photonics and Nanostructures, vol. 3, no. 1, 2005, pp. 30-37

Shu X., Y. Liu, D. Zhao, B. Gwandu, F. Floreani, L. Zhang, and I. Bennion, "Dependence of Temperature and Strain Coefficients on Fiber Grating Type and its Application to Simultaneous Temperature and Strain Measurement," Optics Letters, vol. 27, no. 9, 2002, pp. 701-703.

Sheng H. -J., Fu M.-Y., Chen T.-C., Liu W.-F., and Bor S.-S, “A Lateral Pressure Sensor Using a Fiber Bragg Grating," IEEE Photonics Technology Letters, vol. 16, no. 4, 2004, pp. 1146-1148.

Shevchenko Y.Y., and Albert J., "Plasmon Resonances in Gold-Coated Tilted Fiber Bragg Gratings." Optics Letters, vol. 32, no. 3, 2006, pp. 211-213.

Shevchenko Y.Y., Ianoul A., Chen C., and Albert J., "Realization of Surface Plasmon Resonance Sensors in Standard Optical Fibers," SPIE Photonics North, 2007, to be published.

Sidorenko S., and Martin O.J.F., "Resonant Tunneling of Surface Plasmon-Polaritins," Optics Express, vol. 15, no. 10, 2007, pp. 6380-6388.

Slavk R., Homola J., Ctyroky J., and Brynda E., "Novel Spectral Fiber Optic Sensor Based on Surface Plasmon Resonance," Sensors and Actuators B, vol. 74, no. 1, 2001, pp. 106-111. 
Tseng S. M., Hsu K. Y., Wei H. S., and Chen K. F., "Analysis and Experiment of Thin Metal-Clad Fiber with Index Overlay," IEEE Photonics Technology Letters, vol. 9, no. 5, 1997, pp. 628-631.

Texas Instruments, "On the Sensitivity of Spreeta" [online] Texas Instruments Incorporated, June 2002 [cited July 1, 2007], available form World Wide Web: http://www.sensata.com/files/SPREETA-AppNote-004.pdf

Texas Instruments, "Product Bulletin: TSPR2KXY Biosensor" [online] Texas Instruments Incorporated, 2003 [cited April 18, 2007], available from World Wide Web: http://www.sensata.com/files/spreeta-tspr2kxy-product-bulletin.pdf

Tilley D.R., Basic Surface Plasmon Theory, in 'Surface Plamon Polaritons.' Proceeding of IOP short meetings series, no.9, United Kingdom, 1988, Techno House.

Tubb A.J.C., Payne F.P., Millington R.B., and Lowe C.R., "Single Mode Optical Fiber Surface Plasma Wave Chemical Sensor," Sensors and Actuators B, vol. 41, 1997, pp. 7179.

Tseng S. -M., Hsu K.-Y., Wei H.-S., and Chen K.-F, “Analysis and Experiment of Thin Metal-Clad Fiber with Index Overlay," IEEE Photonics Technology Letters, vol. 9, no. 5, 1997 , pp. 628-631.

Wang S., Boussaad S., Tao N. J., "High-Sensitivity Stark Spectroscopy Obtained by Surface Plasmon Resonance Measurement," Analytical Chemistry, vol. 72, no. 17, 2000, pp.4003-4008.

Wang A.W., Kiwan R., Wite R.M., and Ceriani R.L., "A Silicon-Based Ultrasonic Immunoassay for Detection of Breast Cancer Antigens," Transducers, 1997 International Conference on Solid-State Sensor and Actuators, Chicago. 
Welford K., A Method of Attenuated Total Reflection, in 'Surface Plamon Polaritons' Proceedings of IOP Short Meetings Series, no.9, United Kingdom, 1988, Techno House.

Wendier L., and Haupt R., "Long-Range Surface Plasmon-Polaritons in Asymmetric Layer Structures," Journal of Applied Physics, vol. 59, no. 1, 1986, pp. 3289-3291.

Winnall S. T. and D. B. Hunter, "A Fibre Bragg Grating Based Scanning Receiver for Electronic Warfare Applications," in Microwave Photonics, MWP '01, January 7-9, 2002, pp. 211-214.

Wood R.W., "On a Remarkable Case of Uneven Distribution of Light in a Diffraction Grating Spectrum," in Proceedings of the Physical Society of London, vol. 18, 1902, pp. 269-275.

XANTEC Bioanalytics, "SPR Basics" [online] XANTEC Bioanalytics, [cited July 1, 2007], available from World Wide Web:

http://www.xantec.com/new/index.php?content $=11$

Zhang B., and Kahrizi M., "High-Temperature Resistance Fiber Bragg Grating Temperature Sensor Fabrication," IEEE Sensors Journal, vol. 7, no. 4, 2007, pp. 586591. 